Portland State University

PDXScholar

$1-1-2011$

\title{
High Resolution Measurements near a Moving Contact Line using $\mu$ PIV
}

Jeremiah D. Zimmerman

Portland State University

Follow this and additional works at: https://pdxscholar.library.pdx.edu/open_access_etds Let us know how access to this document benefits you.

\section{Recommended Citation}

Zimmerman, Jeremiah D., "High Resolution Measurements near a Moving Contact Line using $\mu$ PIV" (2011). Dissertations and Theses. Paper 118.

https://doi.org/10.15760/etd.118

This Thesis is brought to you for free and open access. It has been accepted for inclusion in Dissertations and Theses by an authorized administrator of PDXScholar. Please contact us if we can make this document more accessible: pdxscholar@pdx.edu. 
High Resolution Measurements near a Moving Contact Line using $\mu$ PIV

by

Jeremiah D. Zimmerman

A thesis submitted in partial fulfillment of the requirements for the degree of

\author{
Master of Science \\ In \\ Mechanical Engineering
}

Thesis Committee:

Derek C. Tretheway, Chair

Mark M. Weislogel

Raúl Bayoán Cal

Portland State University

(C)2011 


\section{Abstract}

A moving contact line is the idealized line of intersection between two immiscible fluids as one displaces the other along a solid boundary. The displacement process has been the subject of a large amount of theoretical and experimental research; however, the fundamental processes that govern contact line motion are still unknown. The challenge from an experimental perspective is to make measurements with high enough resolution to validate competing theories. An experimental method has been developed to simultaneously measure interface motion, dynamic contact angles, and local fluid velocity fields using micron-resolution Particle Image Velocimetry ( $\mu$ PIV). Capillary numbers range from $1.7 \times 10^{-4}$ to $6.2 \times 10^{-4}$. Interface velocities were measured between $1.7 \mu \mathrm{m} / \mathrm{s}$ and $33 \mu \mathrm{m} / \mathrm{s}$. Dynamic contact angles were manually measured between $1.1 \mu \mathrm{m}$ and $120 \mu \mathrm{m}$ from the contact line, and calculated from $\mu$ PIV data to within several hundred nanometers from the contact line. Fluid velocities were measured over two orders of magnitude closer to the contact line than published values with an increase in resolution of over $3400 \%$. The appearance of a recirculation zone similar to controversial prediction below previously published limits demonstrates the power and significance of the method. 


\section{Acknowledgments}

I would like to thank the following people for their contributions to my education, my graduate research, my happiness, and my future. I couldn't have done it without you.

Derek Tretheway, Mark Weislogel, Raúl Bayoán Cal, Gerry Recktenwald, Alex Baker, Yongkang Chen, Ben Semerjian, Ben Furst, Nathalie Nève, Chris Hinojosa, Drew Wollman, Molly Meyer, Jeff Zimmerman, Leigh Zimmerman, Ken and Lois Wildman, and of course, Cory Bilger. 


\section{Table of Contents}

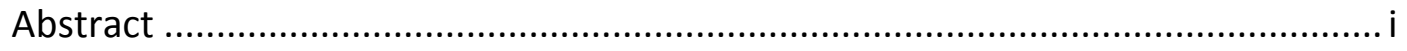

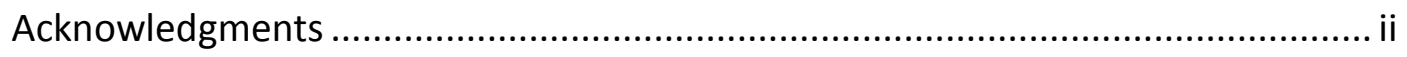

List of Tables ........................................................................................... vii

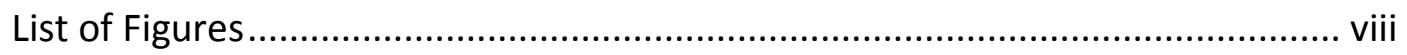

1. Introduction to the Moving Contact Line .................................................... 1

1.1 The Moving Contact Line Problem ....................................................... 1

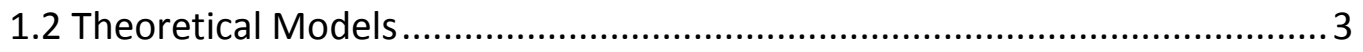

1.2.1 Continuum Hydrodynamic Theory ........................................... 4

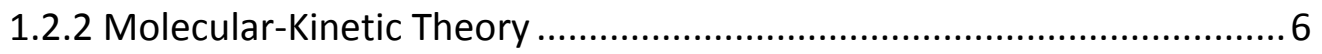

1.2.3 The Microhydrodynamic Theory of Shikhmurzaev ........................... 7

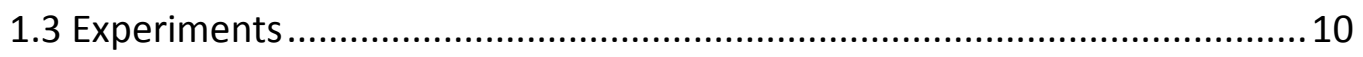

1.3.1 Meniscus Shape and Dynamic Contact Angle ................................. 11

1.3.2 Fluid Motion and Velocity Fields near the Moving Contact Line ..........13

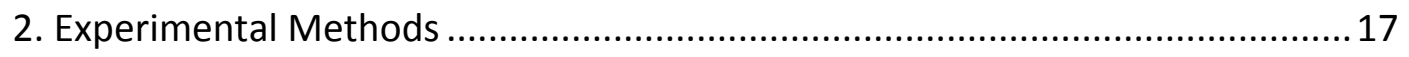

2.1 Micron-Resolution Particle Image Velocimetry ....................................17

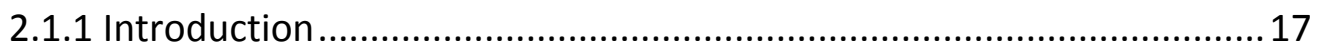

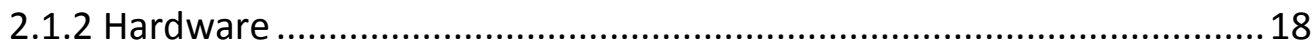

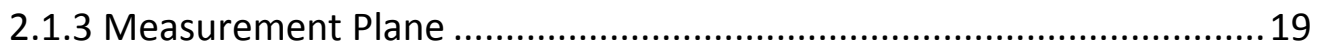




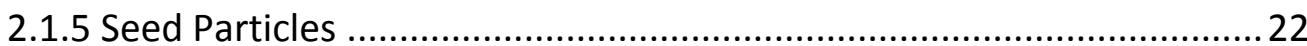

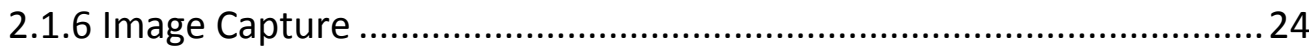

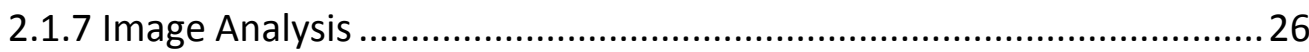

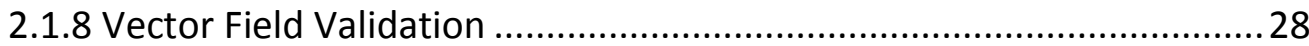

2.1.9 Practical Limits and the Moving Contact Line Problem ..................... 29

2.2 Capillary Flow in a Rectangular Microchannel ........................................30

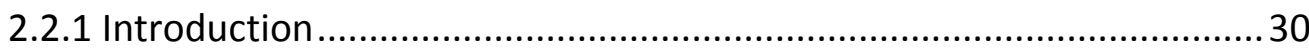

2.2.3 Experimental Channel Design and Specifications .............................32

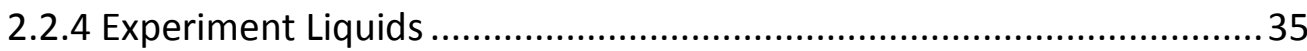

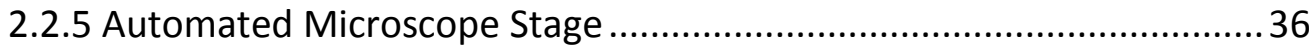

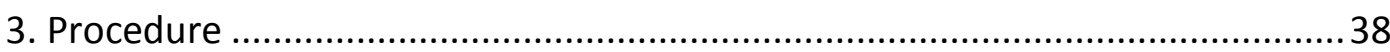

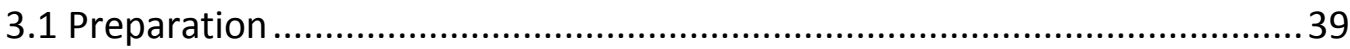

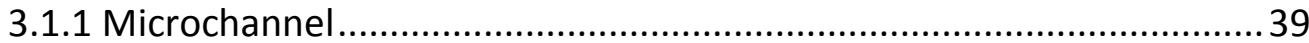

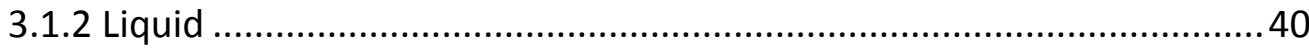

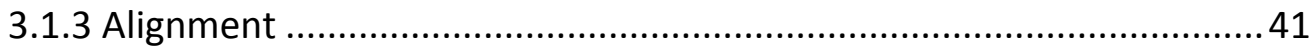

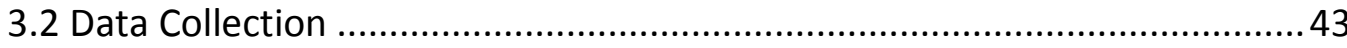

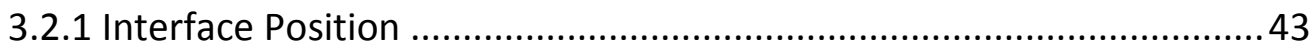




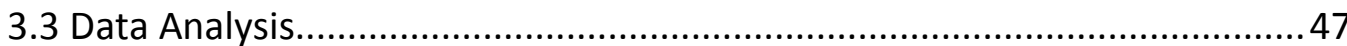

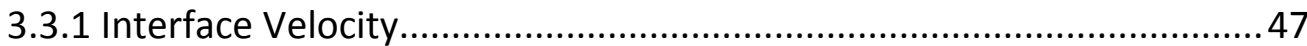

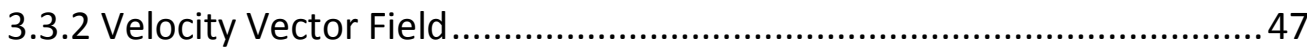

3.3.3 Interface Shape and Contact Angle .................................................50

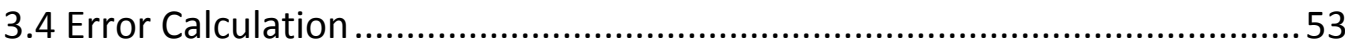

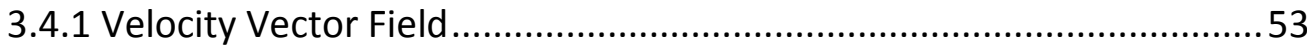

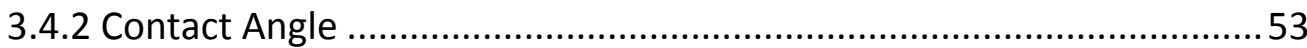

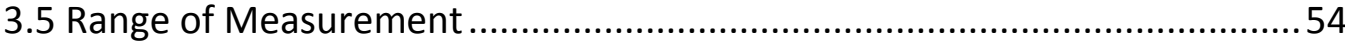

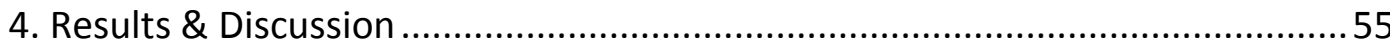

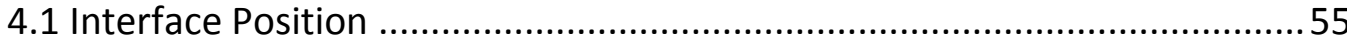

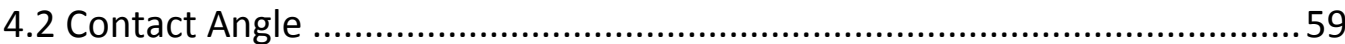

4.3 Fluid Velocities 1 micron from the Moving Contact Line .........................64

4.4 Fluid Velocities < 1 micron from the Moving Contact Line .......................... 70

4.5 Summary of Experimental Results ......................................................... 76

4.5.1 Previous Experimental Limits .......................................................... 76

4.5.2 Apparent Similarity to Theoretical Prediction ...................................77

5. Extended Discussion \& Recommendations ...................................................... 79 


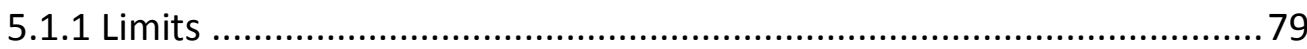

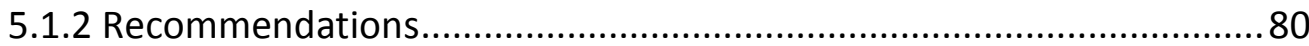

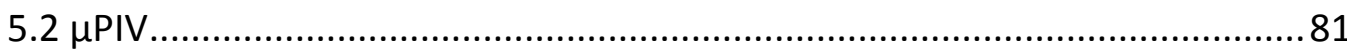

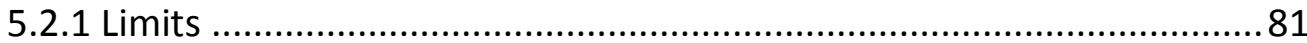

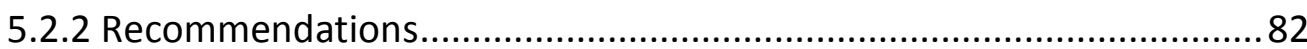

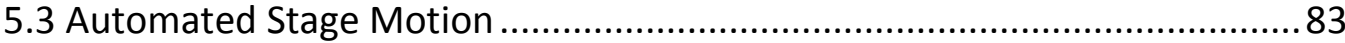

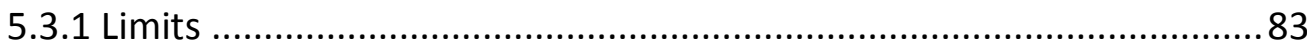

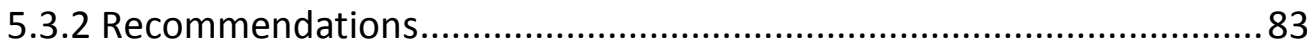

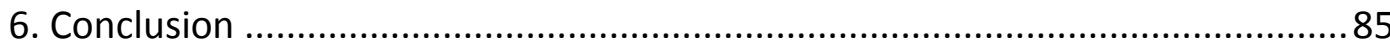

6.1 Overview of Developed Methods................................................................. 85

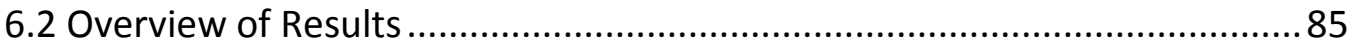

6.3 Contributions to the Study of Contact Line Motion ...................................86

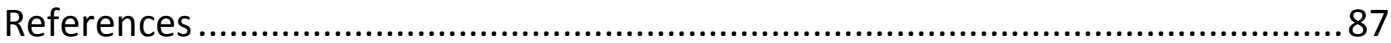

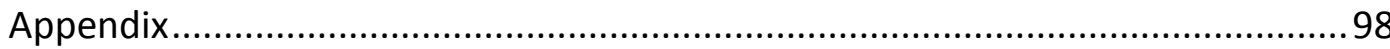




\section{List of Tables}

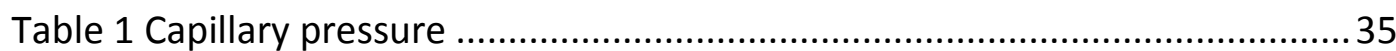

Table 2 Manually measured dynamic contact angles. ....................................61 


\section{List of Figures}

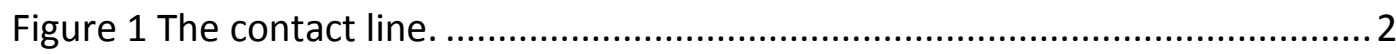

Figure 2 Three length scales of the moving contact line........................................ 5

Figure 3 Molecular Kinetic theory model [Blake 2006].......................................

Figure 4 Shikhmurzaev's "rolling" interface [Shikhmurzaev 1993] ........................ 8

Figure 5 Shikhmurzaev's streamline plot [Shikhmurzaev 2004] ............................ 9

Figure 6 Early experimental techniques [Dussan 1979] .......................................11

Figure 7 Streamline images $1 \mathrm{~mm}$ from the contact line [Savelski 1995]..............14

Figure 8 PIV in PDMS oil $30 \mu \mathrm{m}$ from the contact line [Chen 1997] ...................... 15

Figure PIV $100 \mu \mathrm{m}$ from a submerged glass plate [Fuentes 2005] ........................16

Figure 10 A schematic of the $\mu$ PIV hardware.................................................... 19

Figure 11 The relationship between pairs and valid vectors [Vennemann 2006] .25

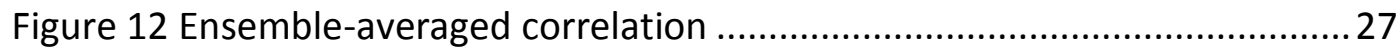

Figure 13 A schematic of capillary flow experiments [Zhu 2010] ...........................32

Figure 14 The microchannel test apparatus (schematic) ...................................... 33

Figure $15 \mu$ PIV image plane at the center of a rectangular microchannel .............33

Figure 16 Stage motion in relation to a moving meniscus ......................................37

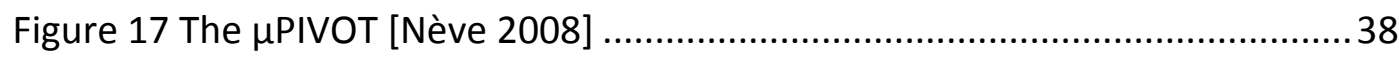

Figure 18 The microchannel test apparatus (photograph) .....................................39

Figure 19 The observed backlit meniscus............................................................ 43 
Figure 20 Insight $3 G$ timing setup window for single image capture mode ..........45

Figure 21 Insight $3 G$ timing setup window for the straddle capture mode...........45

Figure 22 Typical $\mu$ PIV image near the moving contact line ............................46

Figure 23 $\mu$ PIV MATLAB GUI by Steven Wereley (1999, Purdue University) .........48

Figure 24 The velocity vector field in CleanVec .............................................49

Figure 25 Spotlight angle measurement of the backlit interface......................... 51

Figure 26 Spotlight angle measurement between fluorescing particles ..............52

Figure 27 The interface position of GFS in a microchannel (linear fit) .................56

Figure 28 The interface position of GFS in a microchannel (t1/2 fit) ..................56

Figure 29 The interface position of glycerol in a microchannel (linear fit) ...........57

Figure 30 The interface position of glycerol in a microchannel $(\mathrm{t} 1 / 2 \mathrm{fit}) \ldots \ldots \ldots \ldots . . . .58$

Figure 31 Manual contact angle measurement of glycerol using Spotlight ..........59

Figure 32 Manual contact angle measurement of GFS using Spotlight ................60

Figure 33 Dynamic contact angle measurements of GFS using $\mu$ PIV ....................62

Figure 34 Dynamic contact angle measurements of glycerol using $\mu$ PIV .............63

Figure 35 Dynamic contact angle measurements of glycerol using $\mu$ PIV .............64

Figure $36 \mu \mathrm{PIV}$ in glycerol (5.232 $\mu \mathrm{m}$ resolution, $75 \%$ overlap) $\ldots \ldots \ldots \ldots \ldots \ldots \ldots \ldots \ldots . . . . . . . . . . .65$

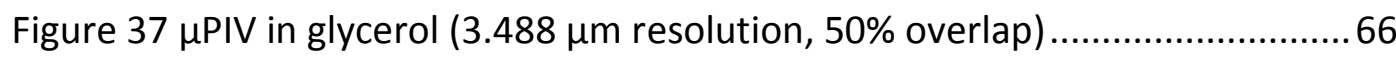

Figure $38 \mu$ PIV in GFS (3.488 $\mu$ m resolution, 50\% overlap) .............................67

Figure $39 \mu \mathrm{PIV}$ in glycerol $(2.616 \mu \mathrm{m}$ resolution, $75 \%$ overlap) ..........................68 
Figure $40 \mu \mathrm{PIV}$ in glycerol $(6.976 \mu \mathrm{m}$ resolution, $50 \%$ overlap) $. . . \ldots \ldots \ldots \ldots \ldots \ldots \ldots \ldots . . . . . . . . . . . . .69$

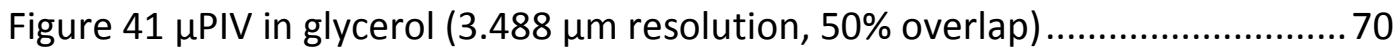

Figure $42 \mu \mathrm{PIV}$ in glycerol (1.744 $\mu \mathrm{m}$ resolution, $50 \%$ overlap) ......................... 71

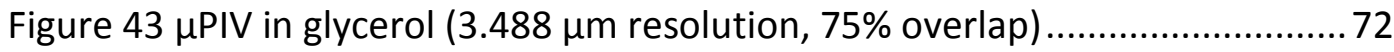

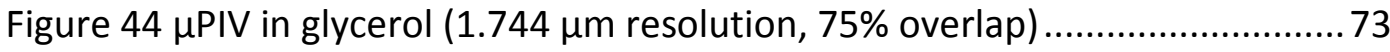

Figure $45 \mu \mathrm{PIV}$ in glycerol $(0.872 \mu \mathrm{m}$ resolution, $75 \%$ overlap) $. . . \ldots \ldots \ldots \ldots \ldots \ldots \ldots . . . . . . . . . . . . . .74$

Figure $46 \mu$ PIV in a magnified region (Figure $44: 1.744 \mu \mathrm{m}$ resolution) ................75

Figure $47 \mu \mathrm{PIV}$ in a magnified region (Figure $45: 0.872 \mu \mathrm{m}$ resolution) ................75

Figure 48 Previous measurement resolutions near the moving contact line ........ 76

Figure 49 Previous measurement resolutions within $30 \mu \mathrm{m}$ of the contact line... 77

Figure 50 Recirculation zone resembles prediction by [Shikhmurzaev 1997] .......78

Figure 51 Corner radius in a "rectangular" glass microchannel ........................... 80

Figure 52 The meniscus in channel with straight and concave walls....................80 


\section{Introduction to the Moving Contact Line}

\subsection{The Moving Contact Line Problem}

A moving contact line is the idealized line of intersection between two immiscible fluids as one displaces the other along a solid surface. A diagram of a contact line is shown in Figure 1. The angle formed between the liquid/gas interface and the solid surface is called the contact angle. Moving contact lines, dynamic contact angles, and the wetting process are found throughout nature and industry. Contact lines appear in our daily lives as water rolls off of a car hood, coffee pours into a cup, clothes absorb water during washing, and our eyes wet by blinking. Understanding the moving contact line is important in coating and painting processes, lubrication, deposition of pesticides on plants, the wetting of powders, and in the design of lowgravity fuel containers and waste processing equipment. Dynamic wetting is important in the design of a Lab-on-a-Chip, a microscale chemical testing device used in medical and environmental applications. To be cost effective and hand-held, Labon-a-Chip devices use passive transport of liquids by tailoring geometric, surface, and fluid properties to exploit wetting dynamics. The moving contact line problem is one of the outstanding challenges in fluid mechanics and has been the subject of research in the fields of Chemistry, Physics, Mathematics, and Mechanical Engineering for nearly a century. 


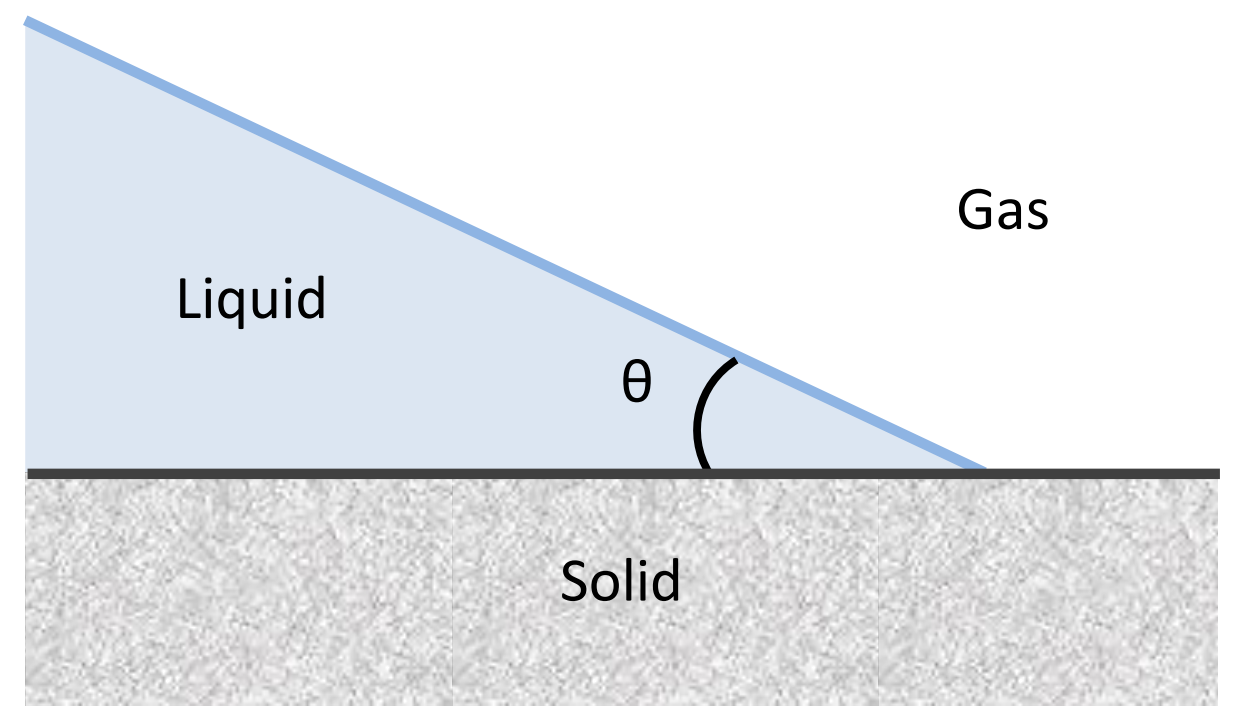

Figure 1 The contact line. $\theta$ is the contact angle.

Because of the ubiquitous nature of the moving contact line, a large amount of theoretical and experimental publications have sought to understand the mechanisms of dynamic wetting. A summary and review of contact line and wetting research over the past several decades has been provided by [Bonn 2009, Blake 2006, Ramé 2002, Kistler 1993, de Gennes 1985, Dussan 1979]. There has been an exponential increase in publications with the keywords "wetting" and "contact line" over the past several decades. This trend is reflected in the results found on the search engines Google Scholar (scholar.google.com/) and Science Direct (www.sciencedirect.com). The number of contact line publications available on Science Direct increased from an average of 125 per year in the 1990 s to over 480 per year since 2005. Similarly, search results on Google Scholar increased from 
approximately 300 per year in 1990 to over 2,000 per year in 2010, with total results over 24,000 as of June 2011. While the contact line has been the subject of a staggering quantity of theoretical, numerical, and experimental research, the fundamental mechanisms that drive contact line motion are either unconfirmed or unidentified. More importantly, contact line motion does not conform to our understanding of classical continuum fluid dynamics.

First described by Huh \& Scriven [1971], a moving contact line leads to a stress singularity when the no-slip boundary condition is applied at the solid surface. When the liquid/gas interface moves along the stationary solid with velocity $U$, the discontinuity at the leading edge yields an unbounded stress requiring an infinite force be exerted to wet the surface. To quote Huh and Scriven: "not even Herakles could sink a solid" [Huh \& Scriven 1971]. It is this contradiction between classic hydrodynamic theory and observation that has piqued the interest of researchers throughout the world.

\subsection{Theoretical Models}

The vast body of theoretical work that attempts to describe and predict contact line dynamics is beyond the scope of this experimental study. For a review of theoretical research on the moving contact line, see [Bonn 2009, Blake 2006, Ramé 2002, Kistler 1993, de Gennes 1985, Dussan 1979]. However, in order to understand 
the motivation for experimental exploration and the important quantities measured, a brief introduction to the basic concepts and a description of the most promising theoretical models is provided.

This work considers only an advancing liquid/gas contact line for flows with low Reynolds number ( $R e \equiv \rho U L / \mu \ll 1$, inertial effects are negligible) and low Bond

number ( $B o \equiv \rho g L^{2} / \gamma \ll 1$, body forces due to gravity are negligible). The most common theoretical models described below also assume that the Capillary number (the non-dimensional ratio of viscous to surface tension forces, $\mathrm{Ca} \equiv U \mu / \gamma$ ) is small $(\mathrm{Ca}<1)$ and that solid surfaces are perfectly smooth. Of particular experimental interest are the model predictions of the apparent and microscopic dynamic contact angles, the fluid/fluid interface velocity, and the local velocity field near the interface.

\subsubsection{Continuum Hydrodynamic Theory}

Continuum hydrodynamic models identify three relevant length scales shown in Figure 2. The geometry-dependent macroscopic outer region is easily observed experimentally and is consistent with classical continuum fluid dynamics. Any changes in dynamic contact angle observed at the macroscale are attributed to viscous dissipation causing viscous bending of the liquid/gas interface in the mesoscopic intermediate region at the limits of traditional optical techniques [Blake 2006]. This intermediate region is independent of geometry and couples the inner 
and outer regions. It is within the inner microscopic region close to the contact line where the microscale physics must lead to changes in observed dynamic contact angles at the macroscale.

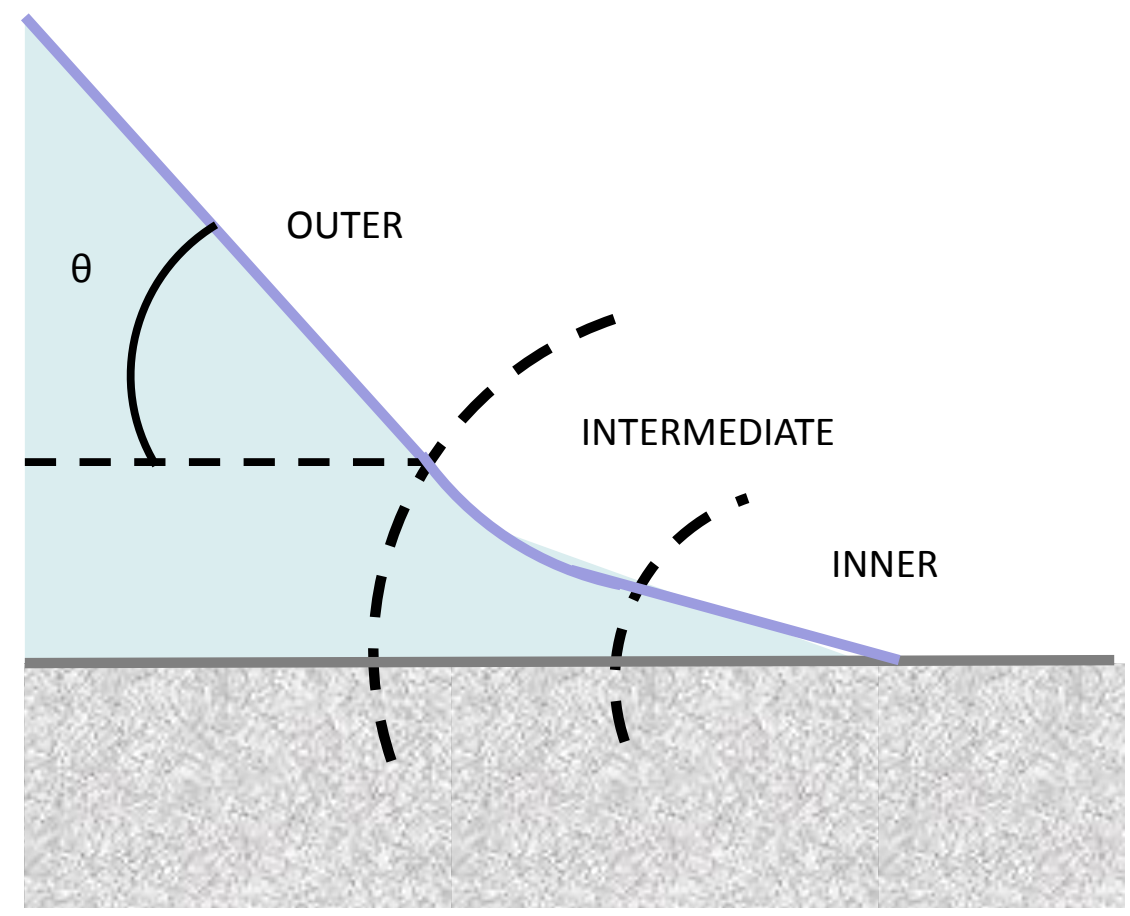

Figure 2 Three length scales identified by Continuum Hydrodynamic theory of the moving contact line.

One approach in hydrodynamic theory has been to truncate the model at the inner region such that discontinuities at the small scale are ignored [Voinov 1976]. This solution is clearly an artificial one in that it does not attempt to describe the fundamental fluid physics that occur in the inner region. A second approach is to relax the no-slip condition near the contact line [Dussan 1979, Cox 1986, Bertozzi 
1998]. This ad hoc slip method allows for non-zero fluid velocities at the liquid/solid interface in the inner region and viscous bending at the liquid/gas interface in order to correct for observed dynamic contact angles. While there is some evidence to support the hydrodynamic approach [Qian 2006], these models break down very close to the contact line as length scales approach microscopic and molecular levels.

\subsubsection{Molecular-Kinetic Theory}

In order to explore the inner region in detail, researchers have modeled the molecular dynamics of individual fluid particles based on molecular-kinetic theory [De Coninck \& Blake 2008]. This approach discards the viscous dissipation of hydrodynamic theory and instead models the attachment and detachment of fluid molecules at the solid surface [Blake 2006]. Figure 3 illustrates the molecular displacement at the leading edge of the moving contact line. The molecular-kinetic model defines a new velocity-dependent microscopic contact angle that is identical to the observed macroscopic angle. This reduces the problem to the molecular scale, where dissipation due to the dynamic friction associated with the moving contact line occurs, and the macroscopic scale where the effects are observed. These molecular models have been used to formulate a continuum hydrodynamic model [Qian 2009] that uses a generalized Navier boundary condition (GNBC) [Qian 2006] with promising results; however it has yet to be experimentally validated. 


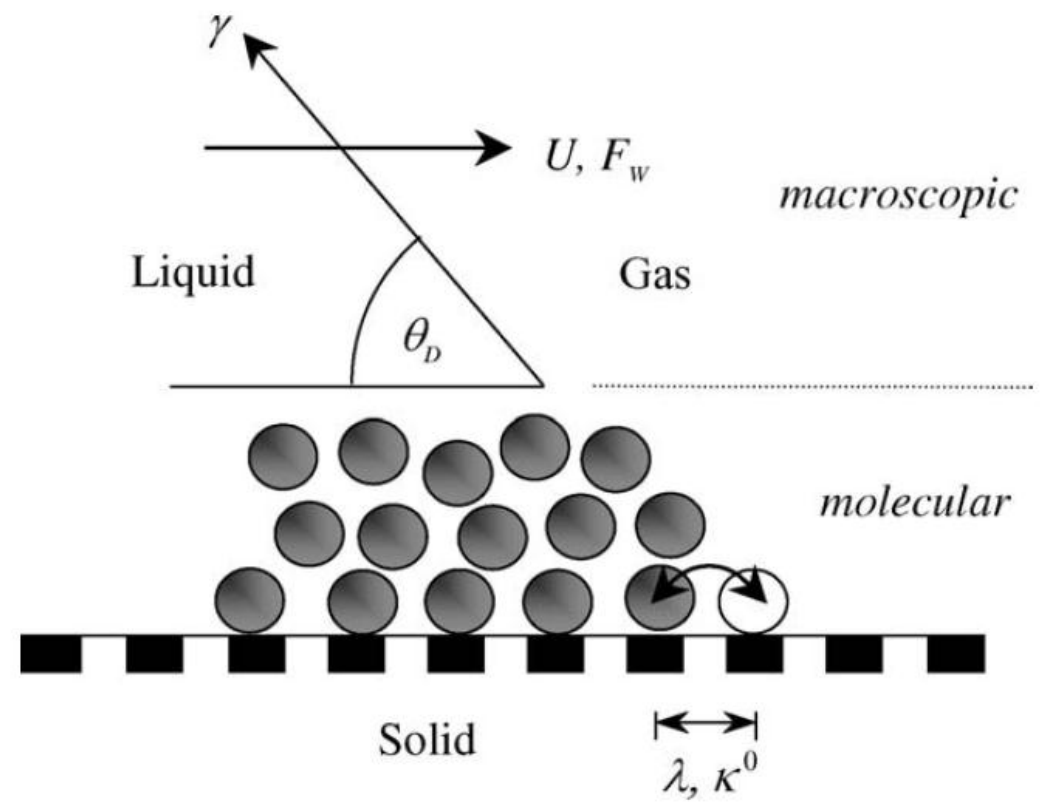

Figure 3 Molecular kinetic theory models molecular attachment and detachment along the solid surface. [Blake 2006]

Additionally, several models combine the hydrodynamic and molecular-kinetic theories [Petrov 1992, Brochard-Wyart \& de Gennes 1992, de Ruijter 1999]. While this approach adds model complexity to capture the governing physics of the moving contact line, the existing combinations rely on a number of fitting parameters with a consequent lack of robustness [Blake 2006].

\subsubsection{The Microhydrodynamic Theory of Shikhmurzaev}

The controversial [Lindner-Silwester 2006, Billingham 2006, Eggers 2004, Shikhmurzaev 2002] theory of Yulii Shikhmurzaev [1993, 1997, 2006] extends the hydrodynamic theory into the microscale by exploiting non-equilibrium 
thermodynamics. The advancing fluid is described as "rolling" and replaces the conventional stagnation point at the contact line with a transfer of liquid from the liquid/gas interface to the liquid/solid interface [Blake 2006] (see Figure 4) [Shikhmurzaev 1993].

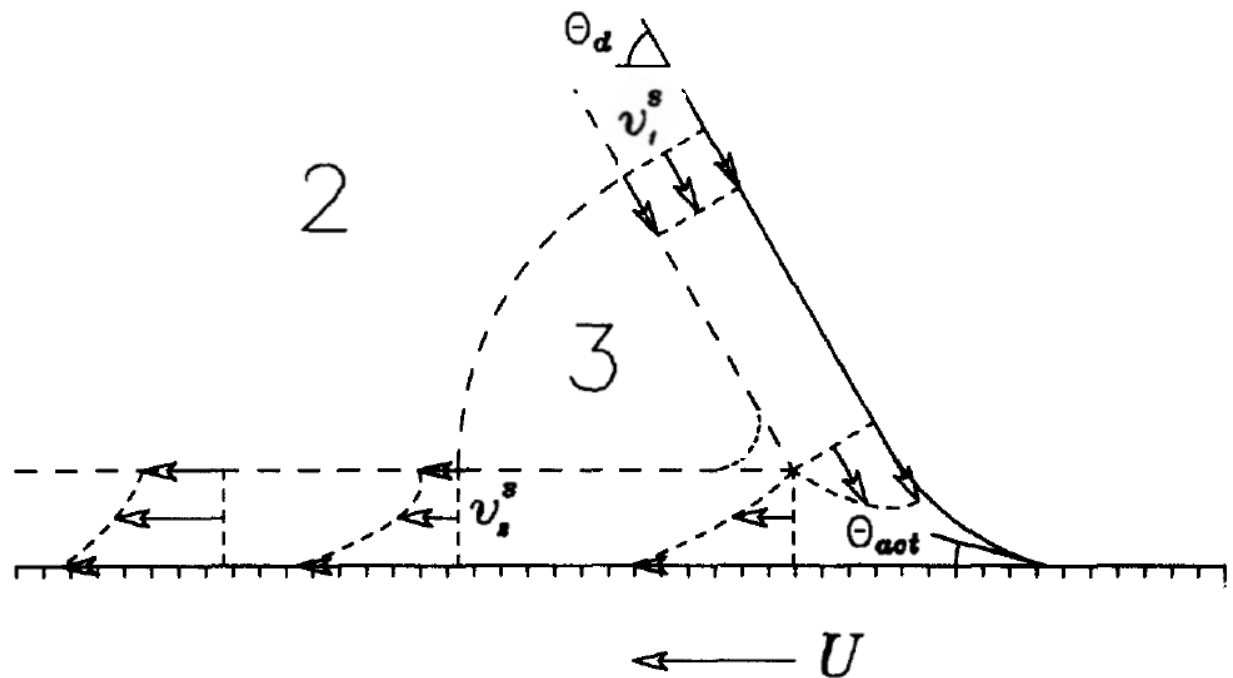

Figure 4 Shikhmurzaev's model describes the advancing fluid as "rolling" with a transfer of liquid from the liquid/gas interface to the liquid/solid interface. [Shikhmurzaev 1993]

Because of the necessary reorganization from one structure to the other, the finite time with which this occurs, and in order to satisfy mass conservation, an interfacial surface tension gradient is imposed. One important characteristic of this model is that the microscopic dynamic contact angle is no longer independent of flow in the inner region but is coupled to the local velocity field. Shikhmurzaev describes three flow regimes similar to continuum hydrodynamic models. However, due to Marangoni flows resulting from temperature gradients caused by shear stresses near 
the leading edge, Shikmurzaev's model predicts the appearance of a small vortex in the inner region shown in the streamline plot in Figure 5 [Shikhmurzaev 1997]. This recirculation zone is unique to Shikhmurzaev's theory and has not been validated by published experimental studies or other theoretical works.
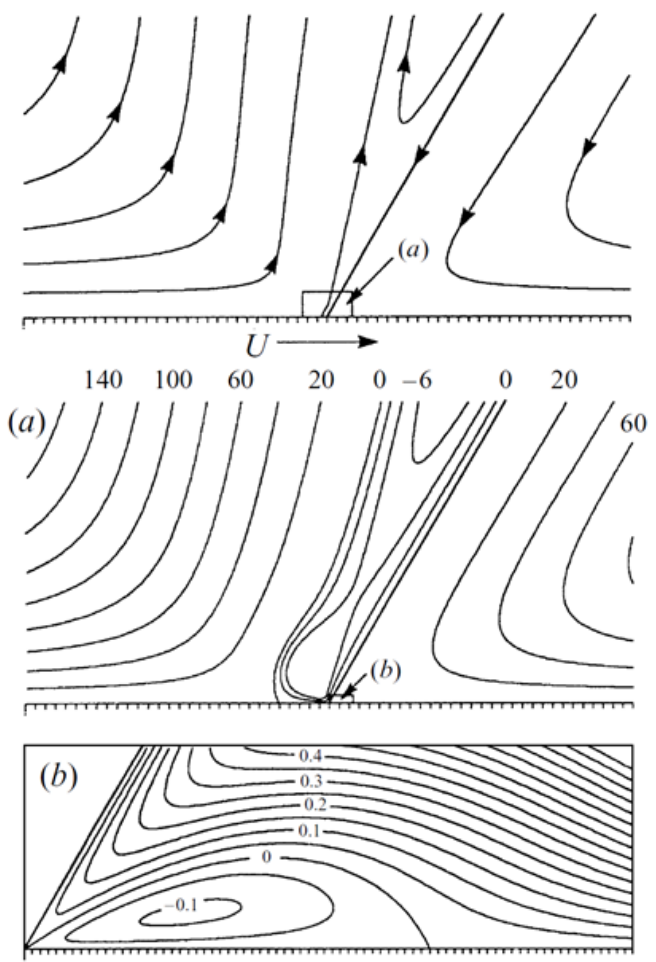

Figure 5 Streamline plot in the three regions of the moving contact line according to Shikhmurzaev. A vortex is predicted in the inner region. [Shikhmurzaev 2004]

The above summary of theoretical models is a mere glimpse into a body of work that has dominated the published literature on the moving contact line problem. Theory has far surpassed the experimental evidence needed to validate competing 
models. That said, significant progress has been made in the experimental measurement of moving contact line parameters.

\subsection{Experiments}

With numerous unconfirmed theoretical models, experimental investigation of the wetting process is required to differentiate between competing theories and ultimately elucidate the fundamental mechanisms of fluid/fluid displacement. A complete model of contact line motion requires accurate measurement of meniscus shape, dynamic contact angle, interface motion, and local velocity fields at all relevant length scales. Scientists have worked to make these important measurements throughout the twentieth century [Dussan 1979, Ralston 2008].

Dussan [1979] summarizes the experimental methods used to quantify contact line motion through the late 1970s. Figure 6 [Dussan 1979] is an illustration of early techniques which include observing displacement in a capillary tube, a spreading drop, a submerged cylinder, a submerged tape, a partially submerged rotating cylinder, and spreading between parallel plates. Dussan groups the experimental techniques into two categories: observation of meniscus shape and contact angle and the observation of fluid motion near the contact line using dyes or particles. These early studies formed the basis of theoretical models; however, the techniques were technologically limited to measurements of the outer region. 
(a)
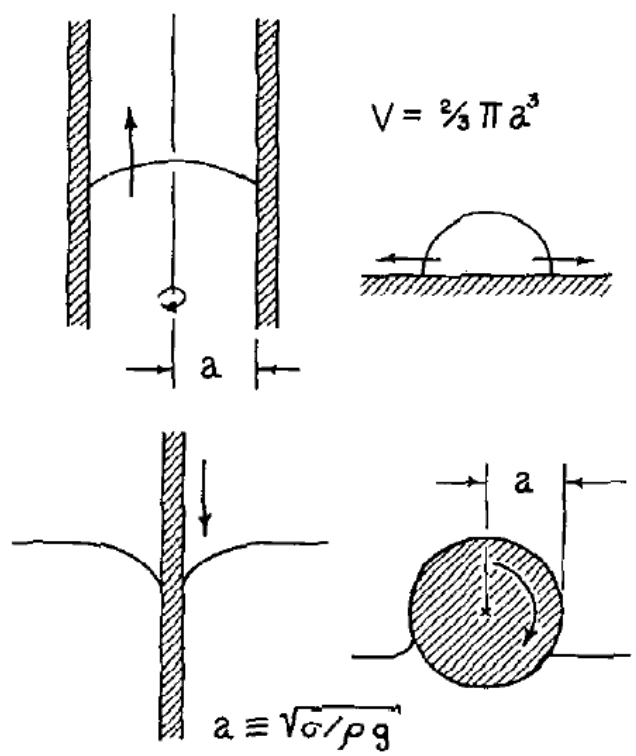

(d) (b)

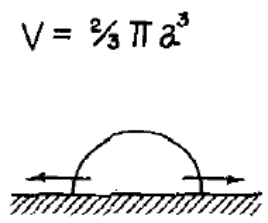

(e) (c)
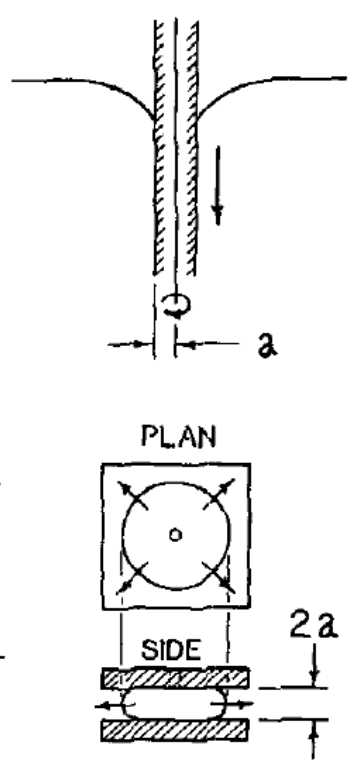

(†)

Figure 6 Early experimental techniques. (a) Capillary rise. (b) Spreading drop. (c) Submerged cylinder. (d) Submerged tape. (e) Rotating cylinder. (f) Spreading between plates. [Dussan 1979]

\subsubsection{Meniscus Shape and Dynamic Contact Angle}

Dussan [1979] concluded that more experiments were necessary to determine the reproducibility of dynamic contact angle measurements and to what extent this value affects the dynamics of the system. In 1991 Dussan, Ramé, and Garoff measured dynamic contact angles approximately $25 \mu \mathrm{m}$ to $250 \mu \mathrm{m}$ from a moving contact line at the surface of a Pyrex tube plunged into a bath of PDMS oil (polydimethylsiloxane) oil. Contact angle measurement precision was within $\pm 0.27^{\circ}$ 
for Capillary numbers $2.8 \times 10^{-4} \leq \mathrm{Ca} \leq 8.3 \times 10^{-3}$. Their experiments showed good agreement with theory and only deviated at the highest speeds (high $\mathrm{Ca}$ ).

In 1993 Marsh, Ramé, and Garoff in a similar experiment to Dussan [1991] measured interface shape and dynamic contact angles within $20 \mu \mathrm{m}$ from the contact line for Capillary numbers $7 \times 10^{-5} \leq C a \leq 2 \times 10^{-2}$. They observed a deviation from theoretical models 10 to $20 \mu \mathrm{m}$ from the contact line which they attributed to optical distortion inherent in the technique.

Using the grating shearing interface technique, by which a deflection pattern is observed as laser light passes through an interface, Shen and Ruth [1998] measured interface shape and dynamic contact angles of paraffin oil displacing air between parallel glass cells for Capillary numbers $4.7 \times 10^{-6} \leq \mathrm{Ca} \leq 2.6 \times 10^{-4}$. Contact angle measurements and interface slope were within $\pm 0.35^{\circ}$ and $\pm 0.25^{\circ}$, respectively, at distances as small as $5 \mu \mathrm{m}$ from the moving contact line. However, similar to [Marsh 1993], these measurements deviated from models less than $10 \mu \mathrm{m}$ from the contact line.

In two studies in 1999 and 2006, in collaboration with Shikhmurzaev, Blake [1999] and Wilson [2006] concluded experimentally that the dynamic contact angle is dependent on contact line speed, local geometry, and ultimately the flow field near the moving contact line. Comparing hydrodynamic slip models to measurements of 
the dynamic contact angle of a curtain coating process (for a wide range of Capillary numbers, $10^{-4} \leq \mathrm{Ca}$ to the point of air entrainment) showed that the dynamic contact angle is not simply dependent on material properties, geometry, and interface speed. This suggests that measurements of dynamic contact angle alone are not sufficient to create or validate a working model of the dynamic wetting process.

\subsubsection{Fluid Motion and Velocity Fields near the Moving Contact Line}

Qualitative observations of fluid flow near a moving contact line have been visualized using dyes, particles on a liquid surface, hydrogen bubbles, and laserDoppler velocimetry [Dussan 1979, Savelski 1995]. The first quantitative measurements of flow patterns in an advancing and receding liquid were published by Savelski et al. in 1995. Streamline images of laser illuminated particles suspended in 10 liquid/fluid combinations were captured approximately $1 \mathrm{~mm}$ from the contact line at the surface of a vertically translating glass plate. Figure 7 provides sample photographs [Savelski 1995]. The results showed qualitative discrepancies with theoretical models, again demonstrating the need for more accurate quantitative measurements of flow fields near the contact line. 

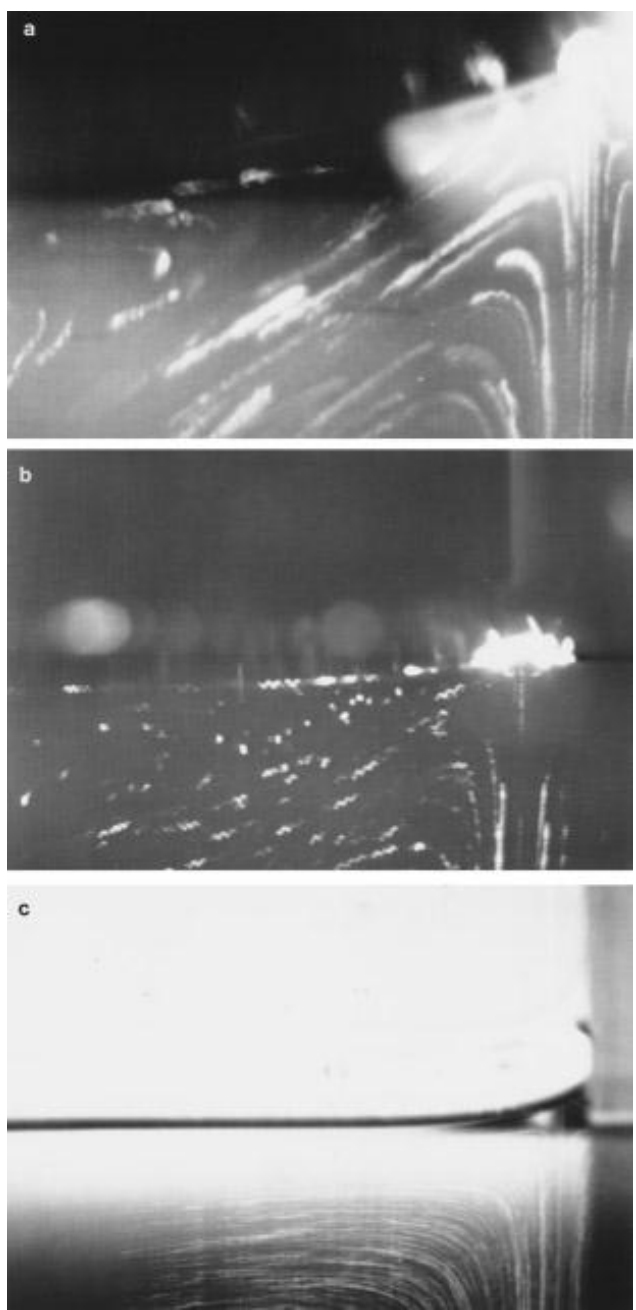

Figure 7 Streamline images of laser illuminated particles approximately $1 \mathrm{~mm}$ from the contact line at the surface of a vertically translating glass plate. [Savelski 1995]

In 1997 Chen, Ramé, and Garoff made simultaneous quantitative measurements of interface shape and the velocity field near a moving interface using particle image velocimetry (PIV). By illuminating dispersed particles $(3 \mu \mathrm{m}<\mathrm{d}<15 \mu \mathrm{m})$ in PDMS oil with a laser light sheet and capturing images near a submerged Pyrex cylinder, velocity vectors were calculated from several hundred microns to as close as $30 \mu \mathrm{m}$ 
from the contact line. Results showed quantitative agreement with theory for Capillary numbers $C a \leq 0.1$ but deviated from $0.1<C a<0.4$ by $\sim 5 \%$. Figure 8 shows a typical velocity field [Chen 1997].

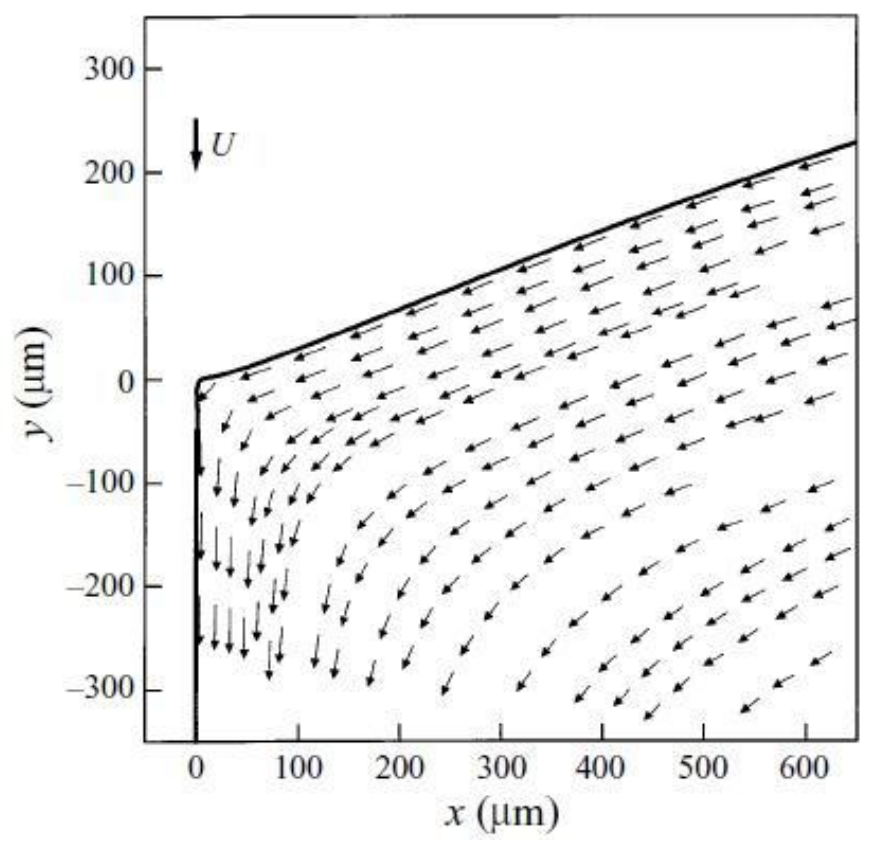

Figure 8 PIV in PDMS oil near a submerged Pyrex cylinder, velocity vectors were calculated from several hundred microns to as close as $30 \mu \mathrm{m}$ from the contact. [Chen 1997]

In 2005, Fuentes and Cerro applied PIV to displaced particles $(\mathrm{d}=12 \mu \mathrm{m})$ near a submerged glass plate in a liquid bath. Figure 9 shows the experimental flow pattern for a Capillary number $\mathrm{Ca}=1.4 \times 10^{-5}$. Fluid velocities were measured at a distance of approximately $100 \mu \mathrm{m}$ from the solid surface and $250 \mu \mathrm{m}$ from the moving contact line. Fuentes and Cerro concluded that there is a close relationship between the forces that shape the contact angle and the forces that shape the flow field near the 
contact line, reiterating the conclusions of [Savelski 1995] and [Chen 1997]. The brief summary of experimental studies reported above represent the current measurement resolution of interface shape and contact angle $(\approx 5 \mu \mathrm{m}$ from the contact line, [Shen 1998]) and fluid motion and velocity field $(\approx 30 \mu \mathrm{m}$ from the contact line, [Chen 1997]). These length-scales are not adequate to elucidate contact line motion within the inner region where current models break down. It is therefore necessary to develop and apply experimental techniques capable of quantifying moving contact line flows at the micron and submicron scales.

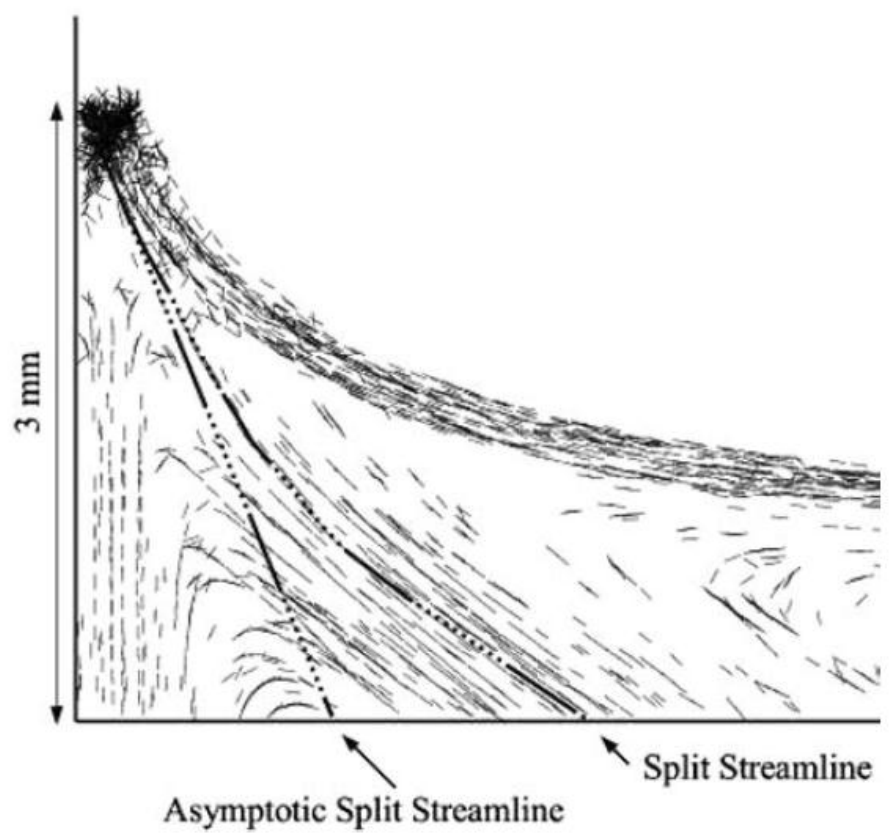

Figure 9 PIV near a submerged glass plate in a liquid bath. Fluid velocities were measured at a distance of approximately $100 \mu \mathrm{m}$ from the solid. [Fuentes 2005] 


\section{Experimental Methods}

\subsection{Micron-Resolution Particle Image Velocimetry}

\subsubsection{Introduction}

Micron-resolution particle image velocimetry ( $\mu$ PIV or microPIV) is a 2-D velocity measurement technique developed in the 1990s by Santiago et al. [Santiago 1998, Meinhart 1998]. $\mu$ PIV was adapted from traditional particle image velocimetry (PIV) in order to quantify and characterize microscale flows in devices common in biomedical, electromechanical, and environmental applications. $\mu$ PIV enables high resolution velocity measurement with approximately $10^{2}$ to $10^{5}$ simultaneous velocity vectors at resolved length scales ranging from $10^{-4}$ meters to $10^{-7}$ meters. The $\mu$ PIV technique uses laser pulses to illuminate fluorescent seed particles in the test fluid. Pairs of images of the displaced particles are captured by a CCD camera. Local velocity is calculated from measured particle displacement, $\Delta x$ at a known time interval, $\Delta t$.

$$
u=\frac{\Delta x}{\Delta t}
$$

A description of the $\mu$ PIV hardware and software as well as a summary of the most important quantitative limits of $\mu \mathrm{PIV}$ measurements are given below. For more 
information and a detailed review of $\mu$ PIV and its applications, see [Lindken 2009, Williams 2010, and Wereley \& Meinhart 2010].

\subsubsection{Hardware}

Figure 10 provides a schematic of the $\mu$ PIV hardware. Pulses of monochromatic light $\left(\lambda=532 \mathrm{~nm}, 3 \times 10^{-9} \mathrm{~s}\right.$ to $5 \times 10^{-9} \mathrm{~s}$ duration, with a maximum energy of $50 \mathrm{~mJ}$ per pulse) from a pair of Nd:YAG (neodymium-doped yttrium aluminum garnet) frequency doubled lasers (Solo III, $15 \mathrm{~Hz}$, New Wave Research, Fremont, CA) travel through a fiber optic cable, pass through a beam expander and enter the optical port of an inverted microscope (TE2000E, Nikon, Tokyo, Japan). The laser pulses are reflected off of a high pass filter cube $(\lambda>550 \mathrm{~nm})$ into the high numerical aperture (NA = 1.45) oil immersion 60x objective lens (PlanApo TIRF, Nikon, Tokyo, Japan). The laser light is focused into the microfluidic device where the test fluid is seeded with fluorescent nanoparticles ( $d=275 \mathrm{~nm}$, nile red, carboxylate-modified polystyrene, Molecular Probes, Carlsbad, CA) with an excitation wavelength of $\lambda=535 \mathrm{~nm}$. The test fluid is volume illuminated by the laser light. The excited particles emit light with wavelength $\lambda=575 \mathrm{~nm}$. The emitted light is collected by the objective lens, passes through the filter cube, and is focused onto the sensor of an interline transfer charge coupled device (CCD) camera (PIVCAM 14-10 Model 630055, TSI, Shoreview, MN) 
with a resolution of 1 megapixel (1376 pixels $x 1024$ pixels) and a physical pixel size of $6.54 \mu \mathrm{m}$. The captured image pairs are sent to a computer for analysis.

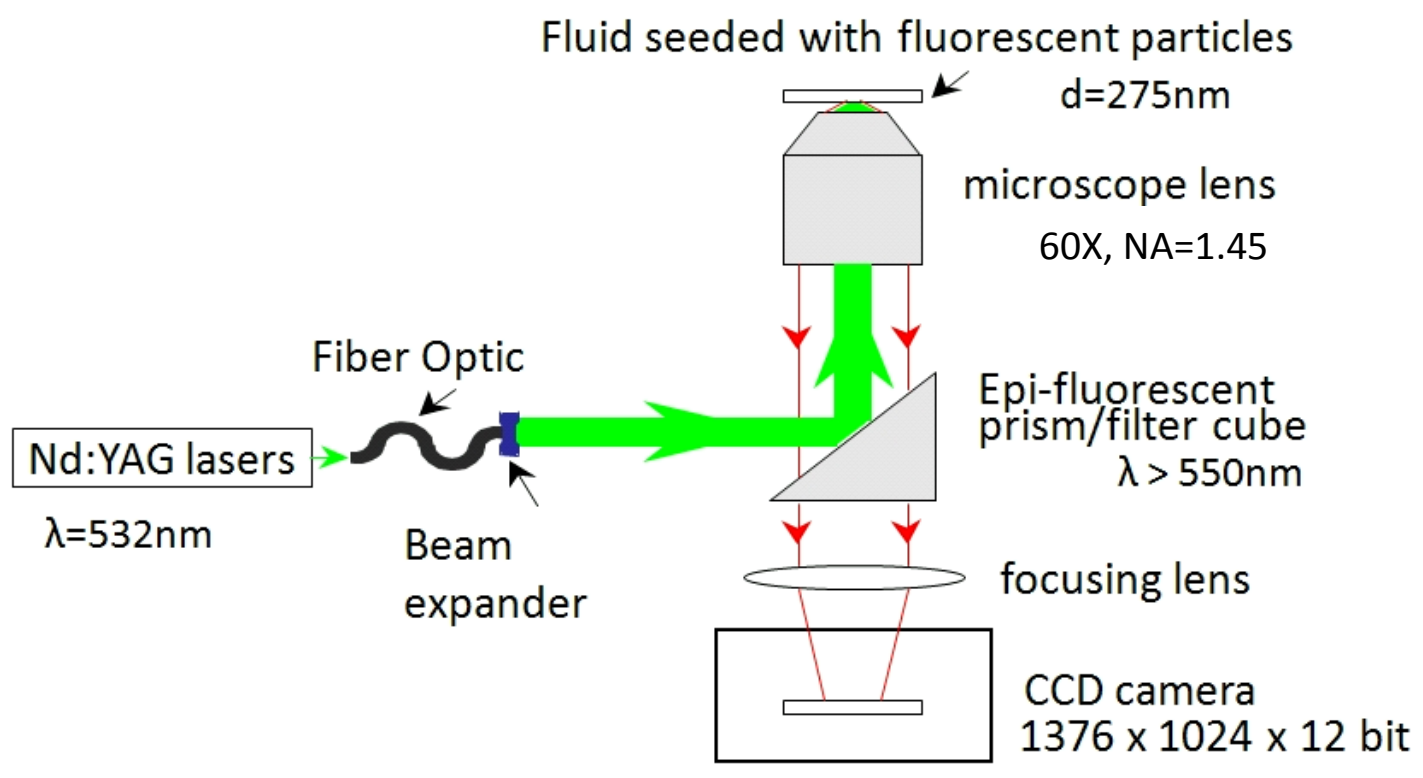

Figure 10 A schematic of the $\mu$ PIV hardware.

\subsubsection{Measurement Plane}

In traditional particle image velocimetry, the 2-D measurement plane is defined by the thickness of the laser sheet used to illuminate the seed particles. In $\mu$ PIV, which uses volume illumination to excite fluorescent seed particles, the measurement plane is defined by the depth of field of the microscope objective lens. More specifically, the seed particles that contribute to the velocity measurements fall inside the depth of correlation, $z_{\text {corr }}$ [Bourdon 2006]. 


$$
z_{c o r r}=2\left\{\frac{(1-\sqrt{\varepsilon)}}{\sqrt{\varepsilon}}\left[\frac{n_{0}^{2} d_{p}^{2}}{4 N A^{2}}+\frac{5.95(M+1)^{2} \lambda^{2} n_{0}^{4}}{16 M^{2} N A^{4}}\right]\right\}^{1 / 2}
$$

where $d_{p}$ is the seed particle diameter, NA is the numerical aperture, $\mathrm{M}$ is the objective magnification, $\lambda$ is the emission wavelength, and $n_{0}$ is the index of refraction of the test fluid. $\varepsilon$ is a threshold weighting function value, (normally 0.01) where particle contribution to velocity calculations is negligible. For a summary of depth of field and correlation depth calculation and validation, see [Meinhart 2000, Olsen \& Adrian 2000, Bourdon 2004]. For typical hardware and particle values with $n_{0}=1.33$ (water), $d_{p}=275 \mathrm{~nm}, N A=1.45, \lambda=575 \mathrm{~nm}, M=60 \mathrm{x}$, and $\varepsilon=0.01$, the depth of correlation is $z_{\text {corr }}=1.95 \mu \mathrm{m}$. The depth of correlation for the test fluids glycerol and glucose/fructose solution (GFS) is $2.36 \mu \mathrm{m}$ and $2.47 \mu \mathrm{m}$, respectively.

While oil immersion objective lenses with a high numerical aperture provide a shallow depth of field and high resolution images, they have a relatively small working distance, $w_{d}$. This introduces an upper limit to channel dimension. The working distance of an objective lens is the deepest point of focus in a sample from the surface of the lens as described by [Meinhart \& Wereley 2003].

$$
w_{d}=\frac{n_{i}}{n_{0}} l_{0}+l_{i}
$$


where $n_{i}$ is the refractive index of the immersion oil, $l_{0}$ is the distance from the surface of the lens to inside surface of the test chamber (microchannel or coverslip), and $l_{i}$ is the depth into the test fluid.

\subsubsection{Resolution}

The resolution of $\mu$ PIV is limited by the diffraction limits of the microscope optics and the ability to resolve the emitted light from the small tracer particles. $\mu$ PIV systems use infinity-corrected optics in order to allow for filters and mirrors to be inserted in the optical path without distorting the image. The diffraction limited spot size is the minimum diameter of the beam of light focused onto the CCD camera sensor. The expression for diffraction-limited spot size, $d_{s \infty}$, for infinity-corrected optics was developed by Meinhart \& Wereley [2003].

$$
d_{s \infty}=1.22 M \lambda\left[\left(\frac{n_{i}}{N A}\right)^{2}-1\right]^{1 / 2}
$$

For the hardware and particle configuration described above, with $M=60 \mathrm{x}, \lambda=575$ $\mathrm{nm}, n_{i}=1.515$, and $N A=1.45$, the diffraction limited spot size is $d_{s \infty}=12.7 \mu \mathrm{m}$. When imaging with the CCD camera described above with a physical pixel size of 6.54 $\mu \mathrm{m}$, the effective minimum resolution of a focused object through a $60 x$ objective lens is $212 \mathrm{~nm}$. 


\subsubsection{Seed Particles}

The characteristics of the seed particles used in $\mu$ PIV introduce limits to measurement accuracy and resolution. The primary limitations are due to the particle size. Seed particles must be sufficiently small to accurately follow the path of the fluid. Particles should be approximately two orders of magnitude smaller than the characteristic length of the test channel geometry. The particles' behavior in flow is defined by its characteristic time, $\tau_{p}$, where $\rho_{p}$ is the density of the seed particle, $d_{p}$ is the particle diameter, $\rho_{f}$ is the density of the test fluid, and $v$ is the kinematic viscosity [Melling 1997].

$$
\tau_{p}=\frac{\rho_{p}}{\rho_{f}} \frac{d_{p}^{2}}{18 v}
$$

For the seed particle to follow the fluid accurately, $\tau_{p}$ should be less than the smallest time-scales associated with the flow.

Brownian motion introduces an additional limitation to particle size and $\mu$ PIV accuracy. The random thermal motion of the suspended particles introduces noise into the measurement of particle displacement. The error $\varepsilon_{B}$, due to Brownian motion is given by [Santiago 1998].

$$
\varepsilon_{B}=\sqrt{\frac{2}{\Delta t} \frac{k_{B} T}{3 \pi \mu d_{p}}}
$$


where $k_{B}$ is Boltzmann's constant, $T$ is absolute temperature, $\mu$ is dynamic viscosity, $d_{p}$ is particle diameter, and $\Delta t$ is the time step between subsequent images. The error due to Brownian motion, $\varepsilon_{B}$, should be significantly smaller than the particle displacement associated with motion of the surrounding fluid, $\Delta x$. Ideally, the error is smaller than the smallest resolvable length, or less than one pixel in the captured images (109 $\mathrm{nm}$ for the PIVCAM described above). Experimental values range from $5 \mathrm{~nm}$ to $60 \mathrm{~nm}$.

The diffraction-limited spot size defines the smallest visible object. However, in the case of a fluorescent particle whose emitted wavelength is larger than the particle diameter, the limit is determined by the effective particle diameter, $d_{e}$, also called the particle image diameter, described by Olsen \& Adrian [2000] and Lindkin [2009].

$$
d_{e}=\left[M^{2} d_{p}^{2}+5.95(M+1)^{2} \lambda^{2}\left(\frac{n_{0}}{2 N A}\right)^{2}\right]^{1 / 2}
$$

For typical hardware and particle values, with $M=60 \mathrm{x}, d_{p}=275 \mathrm{~nm}, \lambda=575 \mathrm{~nm}, n_{0}$ $=1.33$ (water), and $N A=1.45$, the effective particle diameter is $d_{e}=42.6 \mu \mathrm{m}$. For the CCD camera described above and the $60 x$ objective lens, the effective diameter of the fluoresced particle is $709 \mathrm{~nm}$. It is important to note that while a fluorescing particle may appear larger than its diameter, [Prasad 1993] demonstrated that the particle image location is determined to within $1 / 10^{\text {th }}$ the particle image diameter if 
the image spreads across 3 to 4 pixels of the CCD sensor. This reduces particle position uncertainty in the example above to approximately $70 \mathrm{~nm}$.

\subsubsection{Image Capture}

$\mu \mathrm{PIV}$ image capture is an automated process requiring the user to input the proper PIV camera exposure time and laser pulse interval $\Delta t$. Exposure time should be short enough to avoid excess background noise, usually apparent in the form of grayed or foggy images. Particles should appear as bright spots on a black background. The laser pulse interval $\Delta t$ is most critical for accurate velocity calculations. Typical particle displacements should be approximately 10 pixels. Setting a long $\Delta t$ will reduce error due to Brownian motion and is appropriate for flows without strong velocity gradients or significant out of plane particle motion. If strong gradients are present, $\Delta t$ must be reduced such that particles remain in the measurement plane between image pairs.

Theoretically, an accurate velocity field may be calculated from a single pair of particle images. In practice however, noise caused by out of focus particles, Brownian motion, high velocity gradients, low seeding density, and three-dimensional particle motion significantly affects the total number of valid vectors. To overcome these challenges, many image pairs are captured and statistically analyzed to reduce the effect of noise. Vennemann et al. [2006] demonstrate the relationship between the 
number of image pairs (resulting in the total sum of correlations) and the percentage of valid vectors for a $64 \times 64$ pixel resolution in Figure 11. From this data we can extend to higher resolutions by considering the reduction of image data associated with a smaller length scale. To increase the resolution by $50 \%$ would decrease the information contained in the corresponding region by $75 \%$, thereby requiring a $400 \%$ increase in the total number of image pairs to achieve the same percentage of valid vectors. Advanced correlation techniques are used in order to maximize the resolution with the fewest number of images.

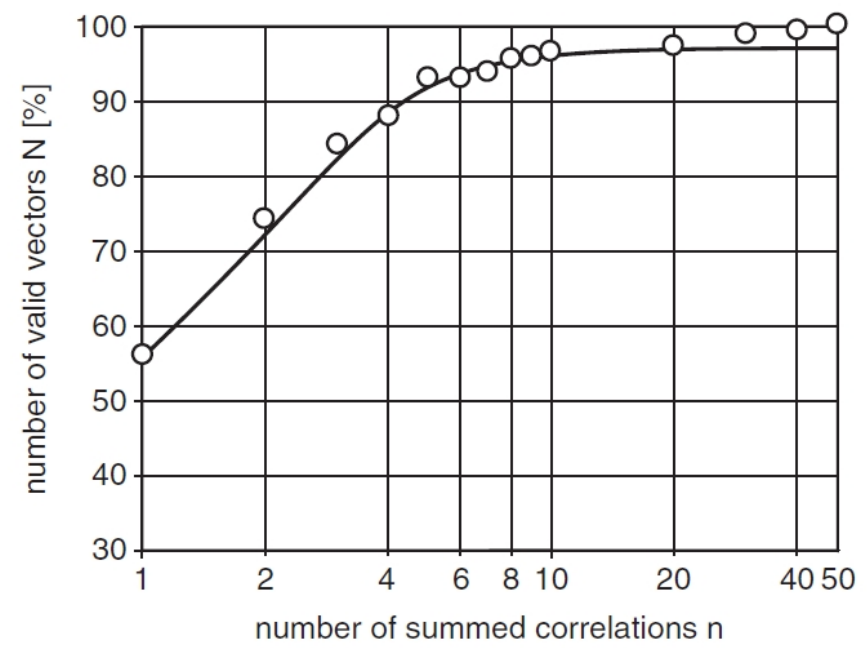

Figure 11 The relationship between the number of image pairs (resulting in the total sum of correlations) and the percentage of valid vectors for a 64 x64 pixel interrogation region. [Vennemann 2006] 


\subsubsection{Image Analysis}

$\mu P I V$ images are analyzed by dividing the images into small interrogation regions. The light intensity (or gray value) in the interrogation region is compared between image pairs to find the most likely displacement of the particles in a process called cross-correlation. The calculated displacement results in a correlation peak in the correlation function, $\Phi_{k}$ [Wereley 2002].

$$
\Phi_{k}(m, n)=\sum_{j=1}^{q} \sum_{i=1}^{p} f_{k}(i, j) \cdot g_{k}(i+m, j+n)
$$

The correlation function $\Phi_{k}$ is the correlation between the $k^{\text {th }}$ image pair where $f_{k}(i, j)$ and $g_{k}(i, j)$ are the gray value distributions of the first and second exposure at an interrogation spot of size $p \times q$ pixels. Depending on the number of particles in the interrogation region, the background noise, and the magnitude of the velocity gradient, several potential peaks or subpeaks may be calculated. To overcome this uncertainty the average image is subtracted (removing background noise) and the correlations are ensemble-averaged.

Assuming that subpeaks are due to random error, only the main intensity peak of the correlation function should appear for all image pair correlations. Therefore, if the correlations are ensemble-averaged, the random correlation peaks tend to zero while the main peak remains. A qualitative example is shown in Figure 12. 

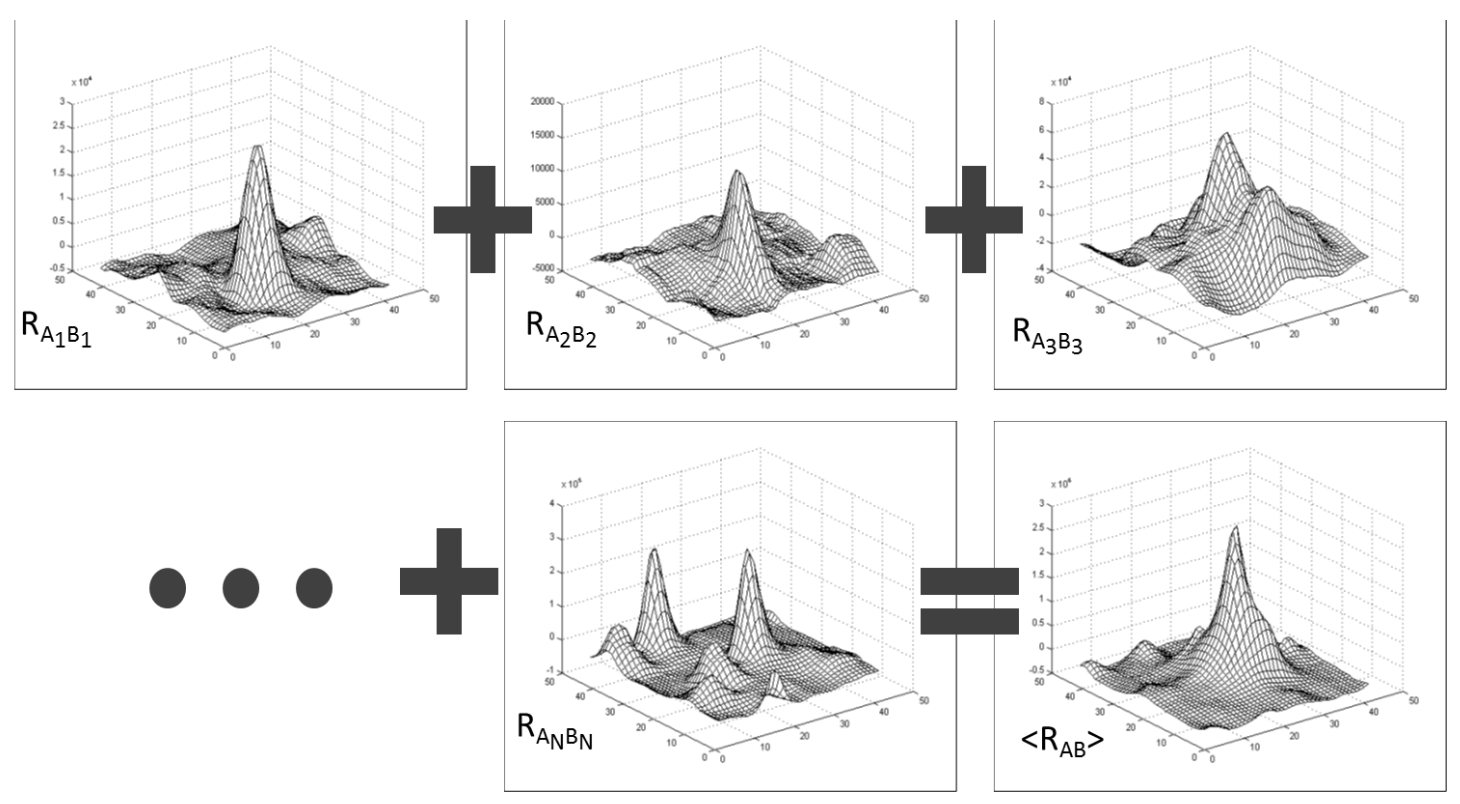

Figure 12 Ensemble-averaged correlation causes the random correlation peaks to approach zero while the main peak remains.

This new ensemble averaged correlation is given by $\Phi_{\text {ens }}$ [Wereley 2002], where $N$ is the number of image pairs.

$$
\Phi_{\text {ens }}(m, n)=\frac{1}{N} \sum_{k=1}^{N} \Phi_{k}(m, n)
$$

The size of the interrogation region determines the resolution of a $\mu \mathrm{PIV}$ velocity field. For example, with a pixel size of $109 \mathrm{~nm}$, a 32 × 32 pixel interrogation region has a resolution of $3.488 \mu \mathrm{m}$. It is desirable to minimize the interrogation size in order to measure flow phenomena on the order of $1 \mu \mathrm{m}$ or less. For flows with particle displacements larger than the desired resolution, two methods are used to process 
images. First, the size of the second interrogation region is increased to be certain to contain the particles from the first interrogation region. This also reduces velocity bias caused by symmetric interrogation regions. The second method is adaptive shifting. By calculating vectors with a larger interrogation region and using the resulting vector field as a best guess for a smaller region, measurement resolution is made incrementally better. However, adaptive shifting can increase the amount of noise if the shift is adapted from bad vectors in the lower resolution data. Invalid vectors must be detected to avoid further errors.

\subsubsection{Vector Field Validation}

While ensemble-averaged correlation drastically reduces the error in velocity vector calculation, additional steps are taken to validate the resulting vector field. Often, the calculated data field will contain vectors that do not fit with the surrounding vector data. This is a result of a random correlation peak exceeding the displacement correlation peak. These invalid vectors are referred to as spurious vectors and are found during post-processing. There are two generally accepted methods of detecting spurious vectors. The first is to generate a scatter plot of velocity data in order to detect vectors with a magnitude outside an expected area. This method is strictly based on the magnitude and does not take into consideration aberrations to vector direction. 
Adapting the median-test used for PIV data, Westerweel et al. [2005] developed a method of universal outlier detection for spurious vectors. This median test quantifies vector field coherence by comparing the $\mathrm{x}$ and $\mathrm{y}$ velocity components $(U$ and $\mathrm{V}$ ) of a vector with the median velocity components of the surrounding vectors. If the flow is continuous and interrogation regions are appropriately sized, the calculated residual, $r$, between the vector and the median should fall below some accepted value depending on flow conditions.

$$
r=\frac{\left|U_{0}-U_{m}\right|}{r_{m}+\varepsilon}
$$

where $U_{0}$ is the displacement vector, $U_{m}$ is the mean of the eight neighboring vectors in a $3 \times 3$ grid, and $r_{m}$ and $\varepsilon$ are normalization terms. $r_{m}$ is the median of $r_{i}=\left|U_{0}-U_{m}\right|$ for $i=1,2,3, \ldots 8$ and $\varepsilon$ is typically set to 0.1 pixels. Westerweel et al. determined that a residual threshold of $r=2$ accurately detected vectors outside the $90^{\text {th }}$ percentile [Westerweel 2005] for flows with Reynolds numbers ranging from $10^{-1}$ to $10^{7}$.

\subsubsection{Practical Limits and the Moving Contact Line Problem}

There are additional practical limitations to measuring microscale flows with $\mu P I V$. Optical access to the flow must be available on at least one side of the device. $\mu$ PIV particles are manufactured and stored in distilled water and therefore test 
fluids must be aqueous or miscible with water in order for the particles to be monodisperse. Fluid flows must be steady (or quasi-steady within the measurement interval) or periodic in nature. However, within the limits described above, $\mu$ PIV measurements are extremely accurate and enable quantitative exploration of the moving contact line problem at a resolution previously unmatched.

\subsection{Capillary Flow in a Rectangular Microchannel}

In order to make measurements of dynamic contact angle, interface velocity, and local flow field velocities with $\mu \mathrm{PIV}$, a flow must be generated in a microscale device that meets all the criteria described above. The selected method is to observe dynamic wetting in a glass microchannel. An introduction to capillary flow in rectangular channels and the experimental design follows.

\subsubsection{Introduction}

Since the invention of micron-resolution particle image velocimetry ( $\mu \mathrm{PIV}$ ) in the late 1990s [Santiago 1998, Meinhart 1998], velocity measurements of liquid flows in rectangular microchannels have been well documented [Meinhart 1999, Lindken 2009, Williams 2010, and Wereley \& Meinhart 2010]. Borosilicate glass microchannels are paired with microscope immersion oil with a matched index of refraction to accurately measure liquid flow near microchannel walls without significant optical aberration. Liquid flows near microchannel walls have been 
measured to within $327 \mathrm{~nm}$ [Tretheway 2002] using hardware comparable to that described above. Considering the highest resolution of published velocity field measurements near a moving contact line $(\approx 30 \mu \mathrm{m}$ from the interface [Chen 1997]), $\mu$ PIV has the ability to increase resolution approximately two orders of magnitude.

The application of $\mu$ PIV to the moving contact line problem has not been reported outside the current work. However, spontaneous capillary flow in corners and in rectangular microchannels has been the subject of theoretical analysis [Ransohoff \& Radke 1988, Weislogel \& Lichter 1998, Ichikawa 2004, Chan 2005, Jong 2007] and has been confirmed experimentally [Dong \& Chatzis 1995, Kim \& Whiteside 1997, Ichikawa 2004, Yang 2004, Chan 2005, Jong 2007, Zhu 2010]. Experiments typically observe flows generated by placing a drop of liquid at an open end of the microchannel. The liquid is drawn into the microchannel by capillary forces. Meniscus shape and location are recorded as a function of time. A schematic of a typical experimental setup is shown in Figure 13 [Zhu 2010]. These studies do not consider the fundamental processes that govern contact line motion and instead provide models to describe the interface motion observed macroscopically. 


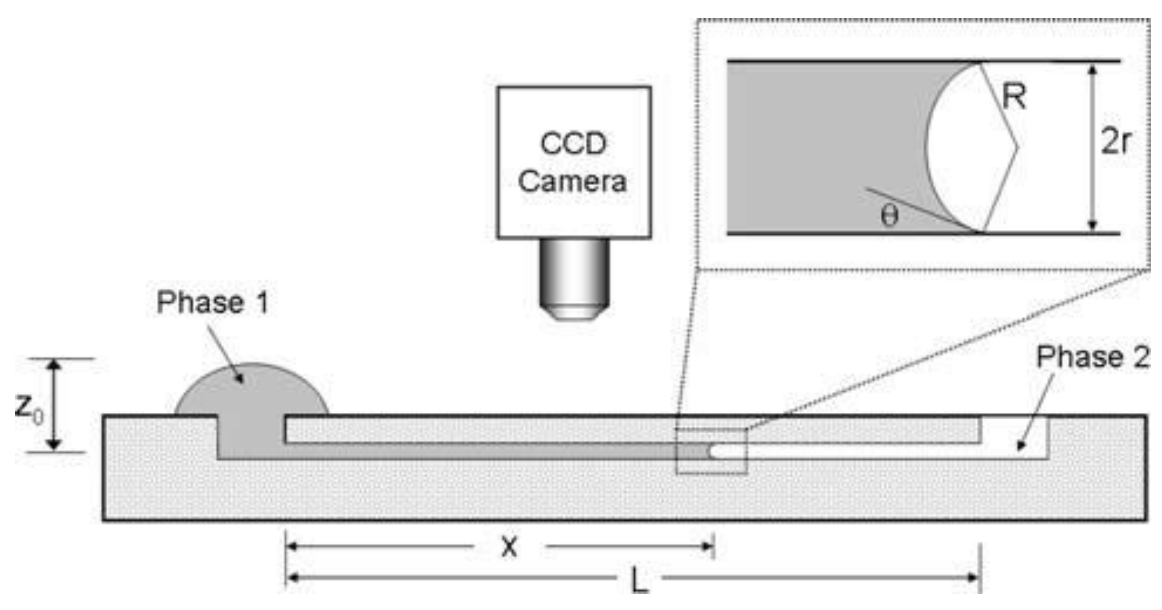

Figure 13 A schematic of a typical capillary flow experimental setup. [Zhu 2010]

Bulk velocity of capillary driven flow in rectangular channels follows predictable functional forms based on static and dynamic contact angles, channel geometry including corner radius, and the liquid/gas viscosity ratio. Capillary flow in rectangular microchannels provides an ideal microscale dynamic wetting process to study experimentally using $\mu$ PIV.

\subsubsection{Experimental Channel Design and Specifications}

Figure 14 is a schematic of the test apparatus. A microchannel is mounted to a glass microscope slide with one end positioned at a reservoir. Fluid velocities are measured near the side wall of the microchannel. Figure 15 provides a schematic of the image plane. By aligning the $\mu \mathrm{PIV}$ image plane with the plane of symmetry at the channel center, the number of particles moving in or out of view is minimized, thereby reducing the noise in the calculated velocities. While a deeper channel will 
also reduce the velocity gradients in the $z$ direction, the working distance of the $60 x$ objective lens limits channel depth to approximately $100 \mu \mathrm{m}$.
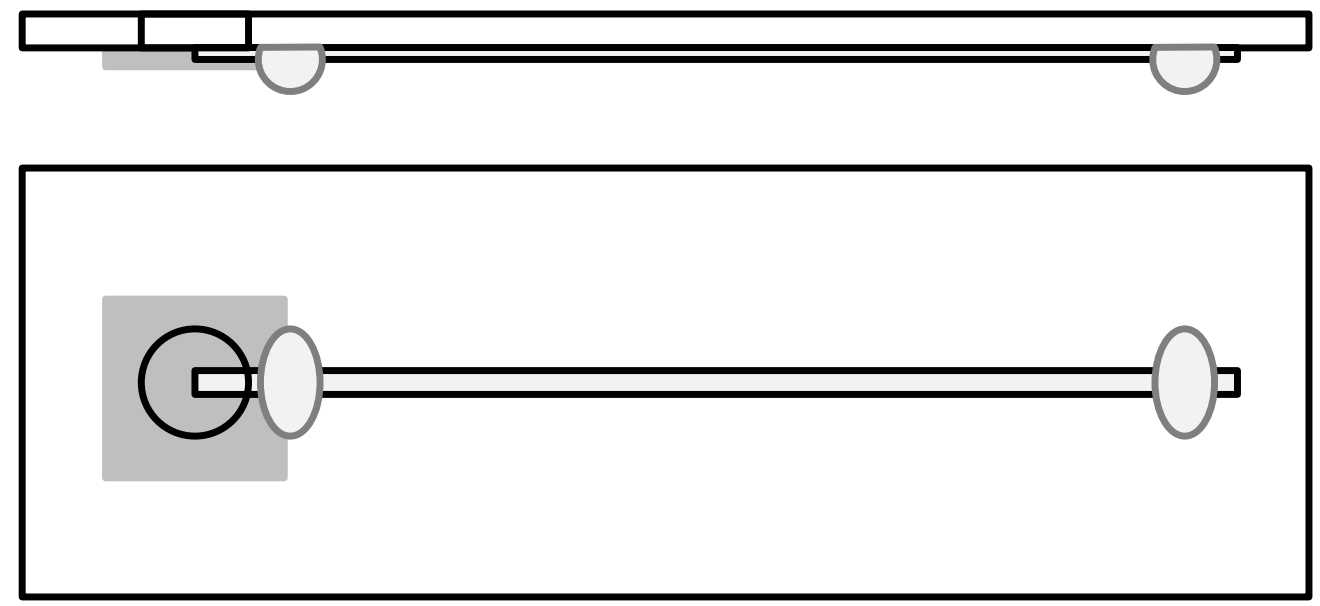

Figure 14 Schematic of the test apparatus. A $5 \mathrm{~cm}$ long glass microchannel is mounted to a $1^{\prime \prime} \times 3^{\prime \prime}$ glass microscope slide with one end positioned at a $5 \mathrm{~mm}$ diameter reservoir.

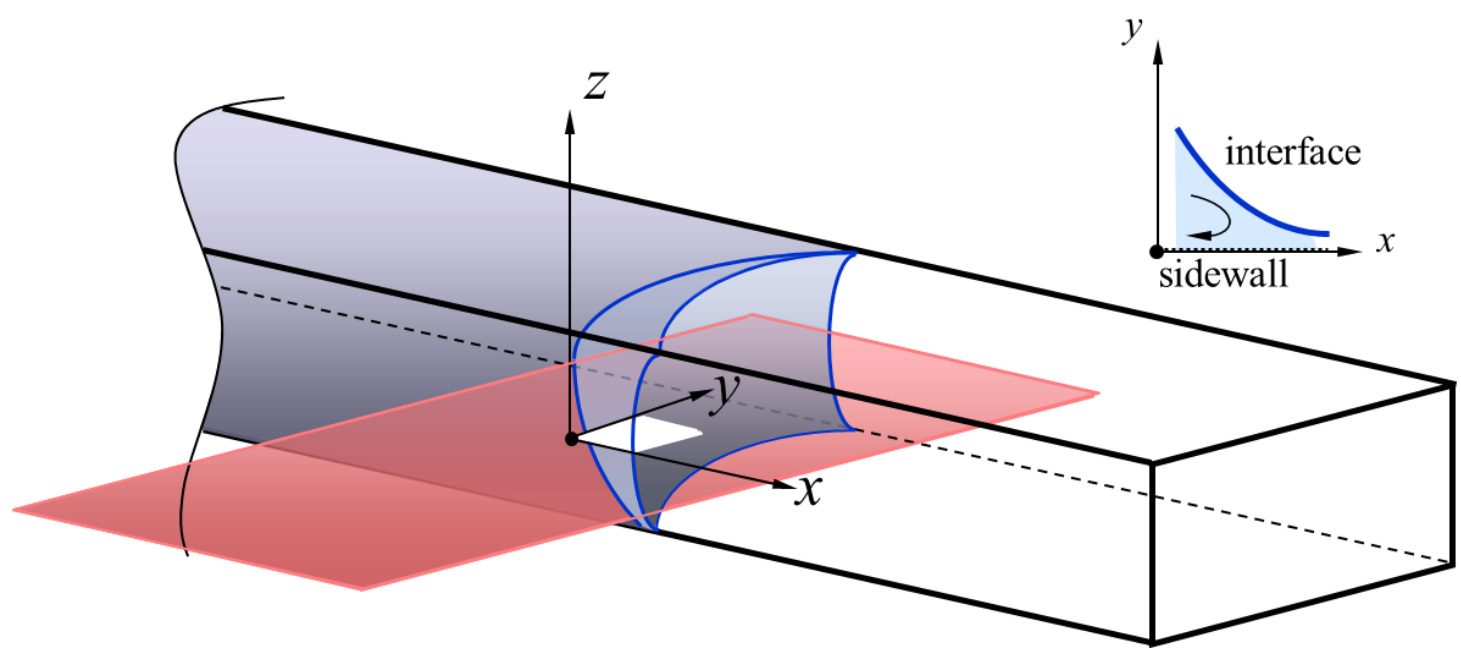

Figure $15 \mu \mathrm{PIV}$ image plane at the center of a rectangular microchannel. 
The dimensions of the reservoir were designed to ensure a negligible change in the capillary pressure difference between the liquid surface curvature at the reservoir and the curvature at the meniscus in the channel over the course of an experiment. The liquid under-fills the cylinder created by the hole in the glass slide. The liquid forms a spherical cap below the surface with a volume $V_{c a p}$ where $h$ is the height of the cap and $\mathrm{a}$ is the radius of the base.

$$
V_{c a p}=\frac{1}{6} \pi \mathrm{h}\left(3 \mathrm{a}^{2}+h^{2}\right)
$$

The curvature of the cap, $R_{\text {cap }}$, is calculated by solving the Pythagorean theorem.

$$
\left(R_{\text {cap }}-h\right)^{2}+a^{2}=R_{c a p}^{2}
$$

The curvature of the cap is used to solve for the capillary pressure, $P_{c, r e s}$, at the reservoir and at the meniscus in the channel, $P_{c, \text { men }}$.

$$
P_{c, r e s}=\frac{2 \gamma \cos \theta}{r} \text { and } P_{c, \text { men }}=2 \gamma \cos \theta\left(\frac{1}{h}+\frac{1}{w}\right)
$$

where $\gamma$ is surface tension, $\theta$ is the contact angle, $r$ is the radius for circular containers, and $h$ and $w$ are the height and width for a rectangular container respectively. To neglect the change in capillary pressure difference between the start of the experiment $\Delta P_{c, i}$ and the end $\Delta P_{c, f}$ when the reservoir has been drained by 
the volume of liquid in the filled microchannel, the percent difference should be $\approx 1$ $\%$ or less.

$$
\% \text { difference }=\left|\frac{\Delta P_{c, i}-\Delta P_{c, f}}{\left(\Delta P_{c, i}+\Delta P_{c, f}\right) / 2}\right| \times 100
$$

Table1 shows the capillary pressures and the percent change in capillary pressure for channel dimensions of $30 \mu \mathrm{m} \times 300 \mu \mathrm{m}, 50 \mu \mathrm{m} \times 500 \mu \mathrm{m}, 100 \mu \mathrm{m} \times 1000 \mu \mathrm{m}$ assuming a $25 \mu \mathrm{L}$ drop is placed into a reservoir with a diameter of $\mathrm{d}=5 \mathrm{~mm}$ and a depth of $1 \mathrm{~mm}$. The percent difference is $\leq 1.21 \%$ for all cases and is considered negligible.

Table 1 Capillary pressure in microchannels at the reservoir and at the meniscus.

\begin{tabular}{|c|c|c|c|c|}
\cline { 2 - 4 } \multicolumn{1}{c|}{} & \multicolumn{3}{c|}{ Capillary Pressure (Pa) } & \multirow{2}{*}{$\begin{array}{c}\text { Percent } \\
\text { Difference (\%) }\end{array}$} \\
\hline $\begin{array}{c}\text { Channel } \\
\text { size }\end{array}$ & Meniscus & Reservoir, initial & Reservoir, final & \\
\hline $30 \times 300$ & 4480 & 12.7 & 14.4 & 0.038 \\
$50 \times 500$ & 2690 & 12.7 & 17.3 & 0.172 \\
$100 \times 1000$ & 1340 & 12.7 & 28.7 & 1.21 \\
\hline
\end{tabular}

\subsubsection{Experiment Liquids}

The aqueous nature of the $\mu$ PIV particle solution limits the fluid type that can be studied with this technique but ensures that the liquids will wet the glass microchannel without surface modification. By changing the viscosity of the test liquids, flows with Capillary numbers ranging from $10^{-6}$ to $10^{-2}$ can be explored. 
Compatible liquids used are glycerol/water and a sugar/water solution comprised of glucose and fructose. By changing the concentration of these liquids in a solution with deionized water, viscosity and contact angle are adjusted. The properties for glycerol at a range of concentrations and temperatures are summarized by Shankar \& Kumar [1994]. The properties of sugar solutions for various concentrations and temperatures are summarized by Telis \& Telis-Romero [2007].

\subsubsection{Automated Microscope Stage}

Even at their most viscous, the test liquids move at velocities much too large to maintain a fixed perspective during an experiment. As ensemble averaging requires a steady flow to obtain a sufficiently large group of image pairs, the interface must remain at a static location in the field of view throughout the experimental measurements. The automated microscope stage (H117 ProScan stage, Prior Scientific, Rockland, MA) moves the microchannel in the opposite direction of the liquid/gas meniscus as it travels along the channel. This effectively freezes the interface in the camera field of view. A schematic of this motion is shown in Figure 16. The stage positioning is accurate to within $10 \mathrm{~nm}$ and the stage velocity ranges from approximately $1 \mu \mathrm{m} / \mathrm{s}$ up to $1 \mathrm{~cm} / \mathrm{s}$. The stage is controlled with a joystick to move with constant velocity or can be controlled via a LabVIEW (National Instruments, Austin, TX) virtual instrument to follow functional forms. In this work 
the stage motion maintains a static interface location for interface velocities between

$1.5 \mu \mathrm{m} / \mathrm{s}$ and $35 \mu \mathrm{m} / \mathrm{s}$ and allows between 50 and 500 image pairs to be captured.

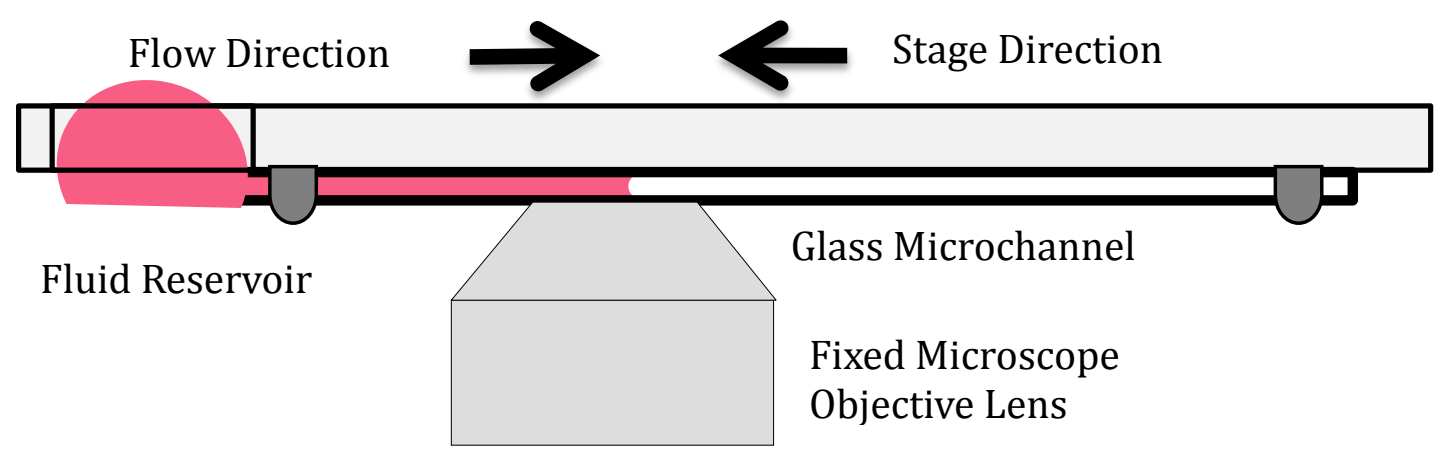

Figure 16 The automated microscope stage moves the microchannel in the opposite direction of the advancing meniscus positioning the interface at a constant location in the field of view. 


\section{Procedure}

Experiments were performed in the Microscale Laboratory at Portland State University. In the Microscale Lab, a novel device called the MPIVOT was developed by Nève et al. [2008]. The $\mu$ PIVOT combines micron-resolution particle image velocimetry ( $\mu \mathrm{PIV}$ ) with optical tweezers (OT). Figure 17 shows a photograph of the MPIVOT. Details and applications of the $\mu$ PIVOT are found in [Nève 2008, Kohles 2009, Nève 2010]. The $\mu$ PIV functionality was used for this experimental study.

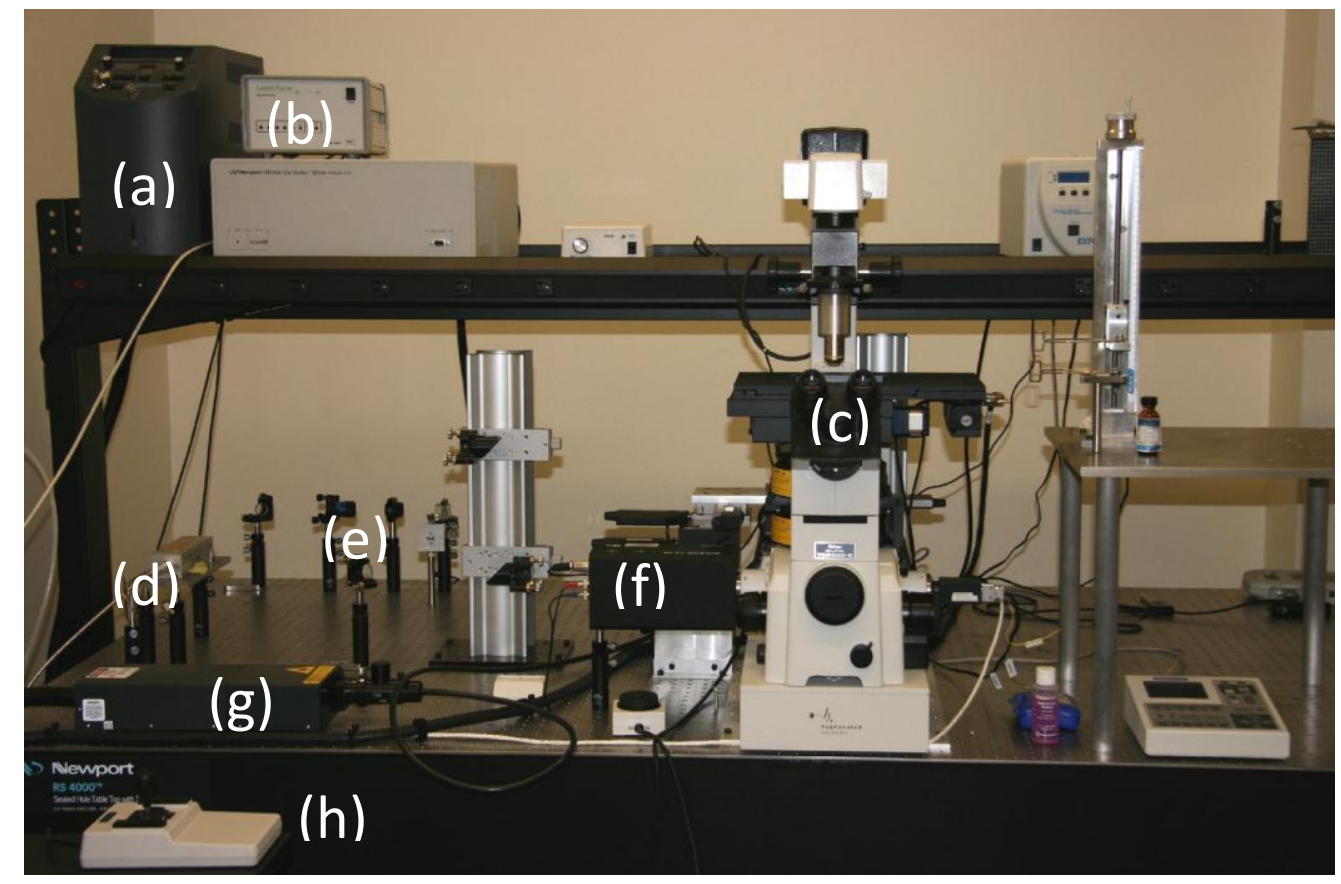

Figure 17 The $\mu$ PIVOT combines micron-resolution particle image velocimetry ( $\mu$ PIV) with optical tweezers (OT). (a) $\mu$ PIV laser power supply. (b) Synchronizer. (c) Inverted microscope. (d) OT laser head. (e) OT light path. (f) $\mu$ PIV camera. (g) $\mu$ PIV laser head. (h) Stage control joystick. [Nève 2008] 


\subsection{Preparation}

\subsubsection{Microchannel}

Figure 18 shows the microchannel test apparatus. Typical channel dimensions (depth $\times$ width) are $30 \mu \mathrm{m} \times 300 \mu \mathrm{m}, 50 \mu \mathrm{m} \times 500 \mu \mathrm{m}$, and $100 \mu \mathrm{m} \times 1000 \mu \mathrm{m}$. An approximately $5 \mathrm{~cm}$ long microchannel (borosilicate, large ratio rectangular, Friedrich \& Dimmock, Millville, NJ) is cut from a 12" length with a razor blade. A $5 \mathrm{~mm}$ diameter reservoir is drilled in a 1" by 3 " glass microscope slide (Fisherfinest 12-5441, Fisher Scientific, Pittsburgh, PA) with a 1/8" ball burr (diamond burr DB12 150 mesh, Daniel Lopacki Co., Cliff, NM) using a low-speed Dremel rotary grinder.

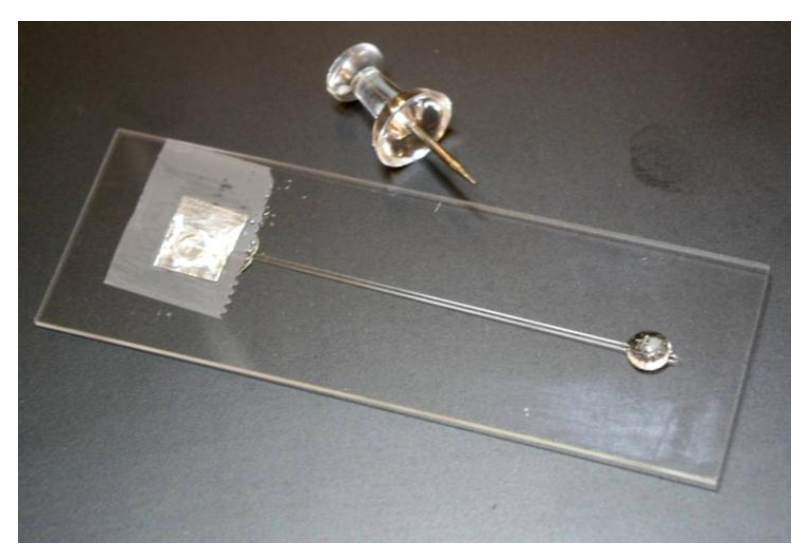

Figure 18 The microchannel test apparatus.

The slide is washed with isopropyl alcohol, rinsed with distilled water, and dried with compressed air. The channel is mounted to the glass microscope slide using a drop of epoxy (5-minute epoxy, Devcon, Danvers, MA) near each channel end. Another slide 
is placed on top of the channel as the epoxy dries to keep the channel in firm, level contact with the surface of the slide. A small square of aluminum foil is taped over the reservoir on the channel side of the glass slide.

\subsubsection{Liquid}

Two types of test fluids were used. A glycerol/water solution and a sugar solution containing glucose and fructose, referred to as GFS. The glycerol is $\geq 99 \%$ pure (Sigma Ultra G6279, Sigma Aldrich, St. Louis, MO). The GFS is an artificial honey that makes use of the stable ratio of glucose ( $\geq 99 \%$ G5400, Sigma Aldrich, St. Louis, MO) and fructose ( $\geq 99 \%$ F0127 Sigma Aldrich, St. Louis, MO) found naturally.

Preliminary experiments showed honey as a suitably viscous fluid for experiments. However, the impurities lead to visual distortion and particle clumping and fluid properties are not consistent. Honey contains $38 \%$ fructose, $31 \%$ glucose, $1 \%$ sucrose, $17 \%$ water, $9 \%$ other sugars, and $3.5 \%$ miscellaneous ingredients. Ignoring the other ingredients, the GFS begins with the ratio of $45 \%$ fructose, $35 \%$ glucose, and $20 \%$ water. $4.5 \mathrm{~g}$ of fructose and $3.5 \mathrm{~g}$ of glucose are mixed with $2.0 \mathrm{~g}$ of distilled water. The solution is put in an oven at $85{ }^{\circ} \mathrm{C}$ until $1 \mathrm{~g}$ of water evaporates. The result is a viscous, homogenous, clear solution compatible with the $\mu$ PIV particles. 
$\mu$ PIV particles (FluoSpheres $0.275 \mu \mathrm{m}$ nile red fluorescent 535/573, Molecular Probes/Invitrogen, Carlsbad, CA) are thoroughly mixed into the test fluid prior to the experiment. The particles are shipped in distilled water with $2 \%$ solids by volume, with added preservatives. $50 \mu \mathrm{L}$ of $\mu \mathrm{PIV}$ particle solution is mixed into $2 \mathrm{~mL}$ of test fluid. This concentration results in approximately 0.0448 spheres per $\mu m^{3}$ or 1 to 4 spheres per interrogation volume in the resulting image plane for 32 pixels $\times 32$ pixels or 64 pixels $\times 64$ pixels, respectively.

\subsubsection{Alignment}

Experiments begin by applying a drop of immersion oil onto the objective lens of the inverted microscope and placing the test channel into position on the stage. The test channel is placed face down with the channel reservoir open at the top. The objective lens is brought into contact with the channel surface and the channel is moved back and forth along the length using the automated stage to coat the surface with immersion oil. The coated channel enables smooth motion during the experiment and a relatively constant volume of oil separating the channel from the surface of the lens. Before the test liquid is dropped into the reservoir, the channel is aligned in all three dimensions.

Channel alignment is one of the most critical aspects of the experimental procedure and often takes an hour or more to reach acceptable precision. The z-axis 
is checked first by focusing on the surface of the channel parallel to the surface of the lens. The stage is translated in the $\mathrm{x}$ and $\mathrm{y}$ directions to confirm a constant location of the surface. If the channel is misaligned in the $z$ direction and the corners of the slide are free from obstruction, the test channel is discarded as the misalignment is most likely due to an improperly mounted channel.

The alignment along the length of the channel is checked by observing the channel side wall as the stage translates forward and backward in the $\mathrm{x}$ direction. If the location of the side wall in the field of view shifts, the slide is gently tapped until it remains parallel to the stage motion. Aligning the channel along the full length to within perceptible limits ensures exceptional alignment during the collection of any particular velocity data set which traverses several millimeters at most.

The final step in channel alignment is to position the focal point at the symmetry plane at the channel center. The channel center is found by focusing on the inside bottom surface of the channel, noting its location in the digital display, similarly finding the top inside surface, and splitting the difference. 


\subsection{Data Collection}

\subsubsection{Interface Position}

$25 \mu \mathrm{L}$ of the test fluid is dropped into the reservoir and a timer is started. The fluid wicks into the channel immediately but the interface is not visible until it moves away from the reservoir tape and foil to the point where the objective lens makes unobstructed contact with the channel surface. The moving interface is positioned with the stage control to a set location in the field of view and the stage position is read from a digital display. Position is recorded to the nearest micron at known times as the liquid travels down the length of the channel. Interface position is recorded concurrently with $\mu$ PIV image capture. Figure 19 is a backlit image of the moving contact line observed through a 10x objective lens.

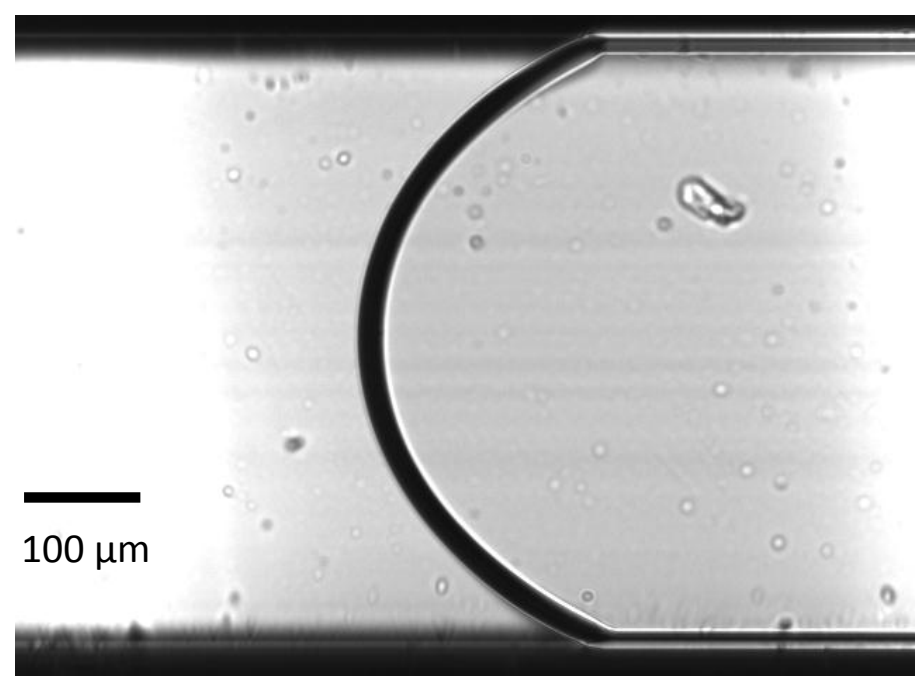

Figure 19 The observed backlit meniscus in a microchannel at 10x magnification. 
During $\mu$ PIV image capture the lights in the laboratory are turned off to reduce background noise in the images. In order to capture backlit images, a shutter is used to allow the microscope lamp to illuminate the sample for a few seconds during the capture of a sequence of images. These backlit images provide a clear view of the interface for contact line measurement, a method to easily determine shift in interface position during $\mu \mathrm{PIV}$ image capture, and a representative image on which the velocity vectors can be superimposed.

\subsection{2 $\mu$ PIV Image Capture}

$\mu$ PIV image capture is controlled with the commercial software package Insight 3G (TSI Incorporated, Shoreview, MN). The exposure is synchronized with the PIV camera to capture a sequence of images. Lasers are set to high power for bright particle fluorescence. The timing setup opens in the window shown in Figure 20 and Figure 21 for single image capture mode and for straddle mode, respectively. The user inputs the pulse repetition rate $(7.25 \mathrm{~Hz}$ and $4.83 \mathrm{~Hz}$ maximum), the laser pulse delay (adjusted for even exposure between pairs), the $\Delta \mathrm{T}$ (typically between 1,000 $\mu \mathrm{s}$ and 15,000 $\mu$ s for optimal displacement), and PIV exposure (for optimal brightness). The timing diagrams shown in Figure 20 (right) and Figure 21 (right) are a graphical representation of the synchronicity between the PIV camera, the laser pulse, and the synchronizer pulse for the single capture and straddle modes. 


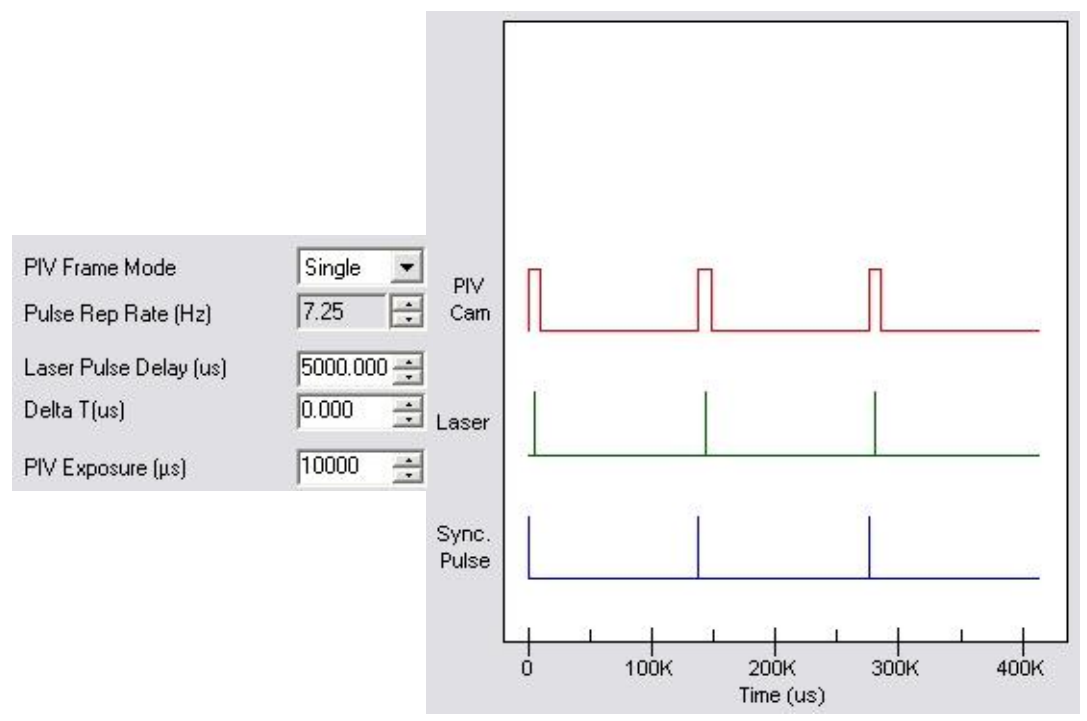

Figure 20 Insight $3 \mathrm{G}$ timing setup window for single image capture mode.

PIV Frame Mode

Pulse Rep Rate $[\mathrm{Hz}]$

Laser Pulse Delay [us]

Delta T(us)

PIV Exposure $[\mu s]$

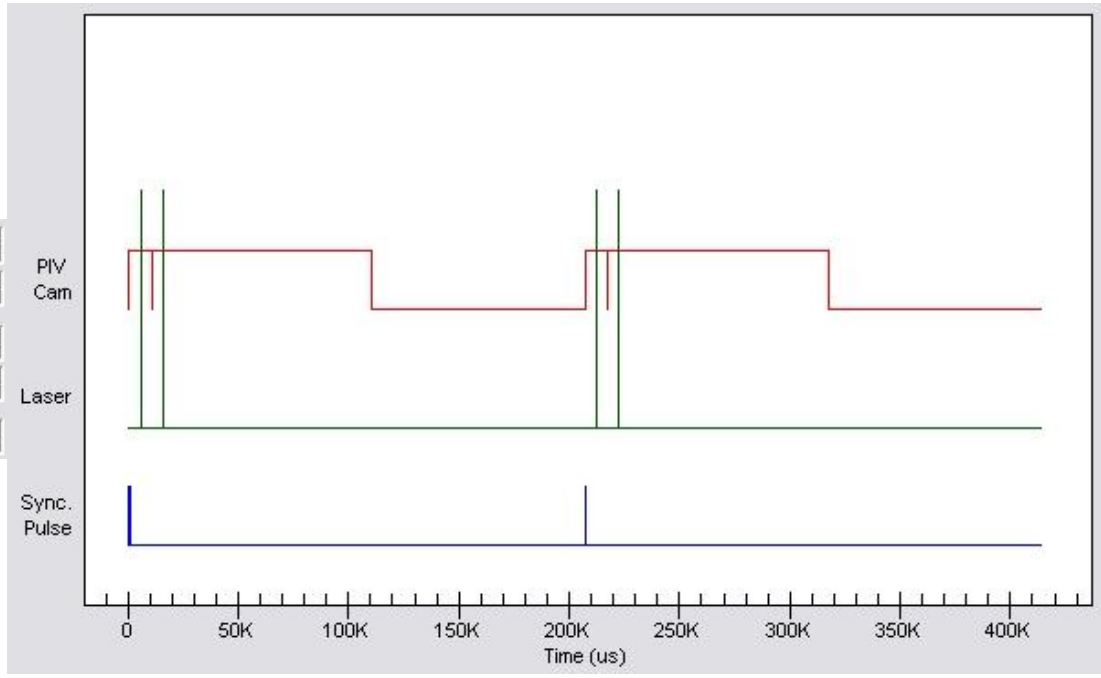

Figure 21 Insight 3G timing setup window for the straddle capture mode. 
Single image capture mode is used for slow flows, where $U \leq 10 \mu \mathrm{m} / \mathrm{s}$. Image capture settings are often determined through trial and error. A sequence of images are captured and then checked qualitatively for clarity. Once settings are properly adjusted, a large number of images may be captured for analysis, typically between 50 and 500 pairs. Figure 22 is a $\mu$ PIV image of the fluorescing particles near the contact line. Capturing multiple image pairs requires the interface be at a constant location in the field of view.

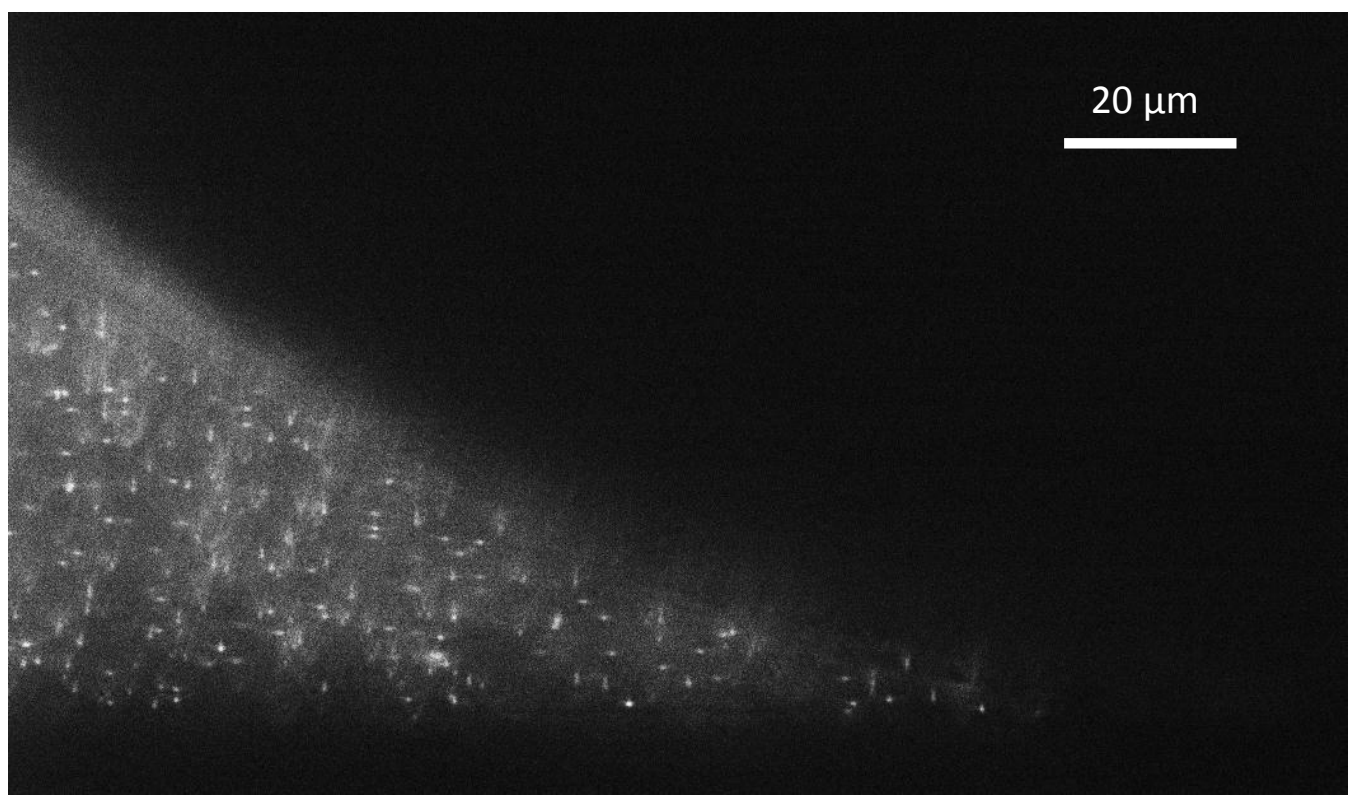

Figure 22 Typical $\mu$ PIV image of the fluorescing particles near the moving contact line.

The stage motion is controlled manually with the joystick. The speed is locked into position by releasing the spring action, allowing fine control of the constant velocity. The speed is adjusted until the interface appears still. At this point the 
images are captured. Backlit images captured at the beginning and the end of the sequence are compared to determine the magnitude of shift in the interface position during the image capture. If this shift is larger than approximately $1 / 10$ of the target interrogation region, a subset of the captured images is selected such that the shift remains nearly imperceptible. This reduction in the number of image pairs analyzed increases the effect of noise inherent in $\mu$ PIV and may result in poor quality data.

\subsection{Data Analysis}

\subsubsection{Interface Velocity}

The position of the interface is plotted with respect to time. The average velocity is obtained from the slope of the linear fit to the position data. This average velocity is useful in approximating the Capillary number $C a \equiv U \mu / \gamma$ and the Reynolds number $R e \equiv \rho U L / \mu$. The coefficient of determination, or the $\mathrm{R}^{2}$ value, of the linear fit is useful to determine the accuracy of the linear approximation of interface motion during image capture.

\subsubsection{Velocity Vector Field}

MPIV data is processed using MATLAB (MathWorks, Natick, MA) PIV code written by Steven Wereley (1999, Purdue University, West Lafayette, IN). Figure 23 is the MATLAB graphical user interface. The calculation of velocity vectors is an iterative 
process by which the resolution is incrementally increased by shrinking the interrogation region. The first pass is typically coarse in order to determine the overall quality of the images. The image pairs are imported into the list. The interrogation size is defined, $64 \times 64$ pixels or $32 \times 32$ pixels for a first run. The percent overlap is set. Typically the percent overlap is $50 \%$. This doubles the number of vectors and decreases the vector spacing, but does not increase the resolution, determined by the size of the interrogation region. 'Subtract Average' and 'Ensemble Average Correlations' are selected. The correlation algorithm is 'A $\rightarrow B, B \rightarrow C, C \rightarrow D$...' for single image capture mode and ' $A \rightarrow B, C \rightarrow D, E \rightarrow F . .$. ' for the more common straddle mode. The peak fit routine is a Gaussian 9-point. A vector file name is defined and the whole field is analyzed.

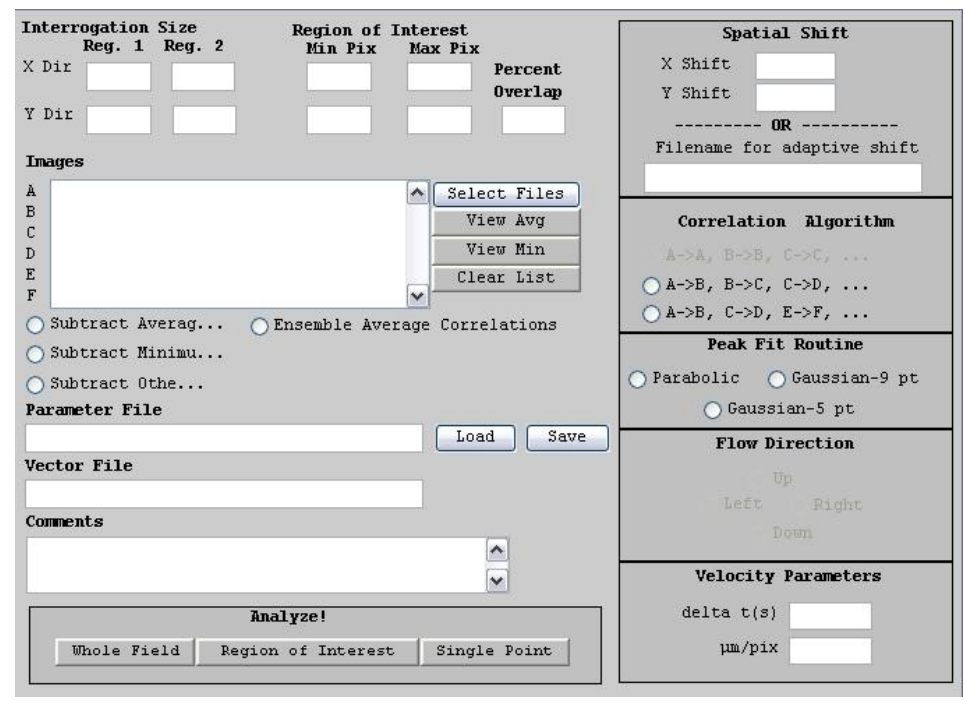

Figure $23 \mu \mathrm{PIV}$ data is processed using code written by Steven Wereley (1999, Purdue University). 
The analyzed vector field data is imported into CleanVec (Steven M. Soloff and Carl D. Meinhart, 1999), a vector analysis tool that allows bad vectors to be removed and fields smoothed. CleanVec provides the first visual representation of the velocity data and is used to make a qualitative conclusion about the collected images. A sample view of an unedited vector field in CleanVec is shown in Figure 24. Vectors outside the liquid region are removed and the results are saved. This removal process is accelerated by removing vectors outside an acceptable magnitude. Vectors from outside the liquid region typically have magnitudes far outside the range of valid velocity vectors. This new vector data can be imported back into the PIV MATLAB code for further refinement using adaptive shifting.

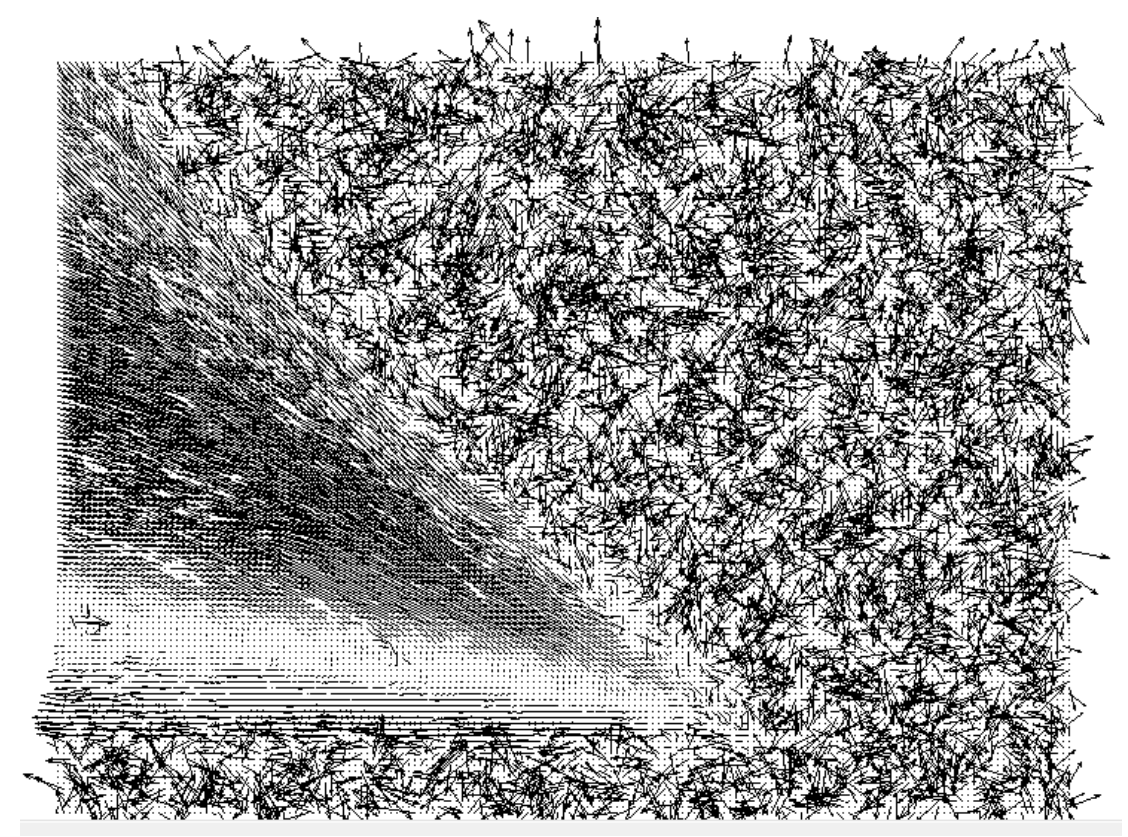

Figure 24 The velocity vector field in CleanVec prior to removing vectors outside the liquid region. 
To apply the adaptive shifting technique, the image pairs are again imported into the MATLAB code. The previously analyzed vector field data is loaded in the 'Filename for adaptive shift'. The same settings are selected but with a smaller interrogation region. This allows the software to use the previous vector data as a best guess and can increase the resolution and reduce the number of spurious vectors. This process may be repeated many times; however, the image quality, fluid velocities, and particle concentration impose limits that will eventually result in noisier vector fields. After removing the vectors outside the liquid region in CleanVec, the data is imported into Excel (Microsoft Corporation, Redmond, WA) for conversion from $\Delta$ pixel $/ \Delta \mathrm{T}$ to $\mu \mathrm{m} / \mathrm{s}$, imported into MATLAB for error calculation and analysis, and finally displayed as a vector plot using MATLAB or TecPlot (TecPlot Inc., Bellevue, WA).

\subsubsection{Interface Shape and Contact Angle}

Interface shape and contact angle data was collected from either backlit images of the interface or $\mu$ PIV particle images. Two methods of measurement (manual and MATLAB) are used. The manual method uses the angle measurement tool of Spotlight (Robert Klimek \& Ted Wright, NASA Glenn Research Center, Microgravity Science Division), image analysis software developed at NASA. Backlit images are imported into Spotlight and the angle tool is positioned from the leading edge at the 
liquid/gas/solid intersection to a point on the liquid/gas interface as shown in Figure 25. Similarly, $\mu$ PIV images are imported and the angle tool is positioned between two focused particles near the interface as shown in Figure 26. This method provides an approximation of the dynamic contact angle.

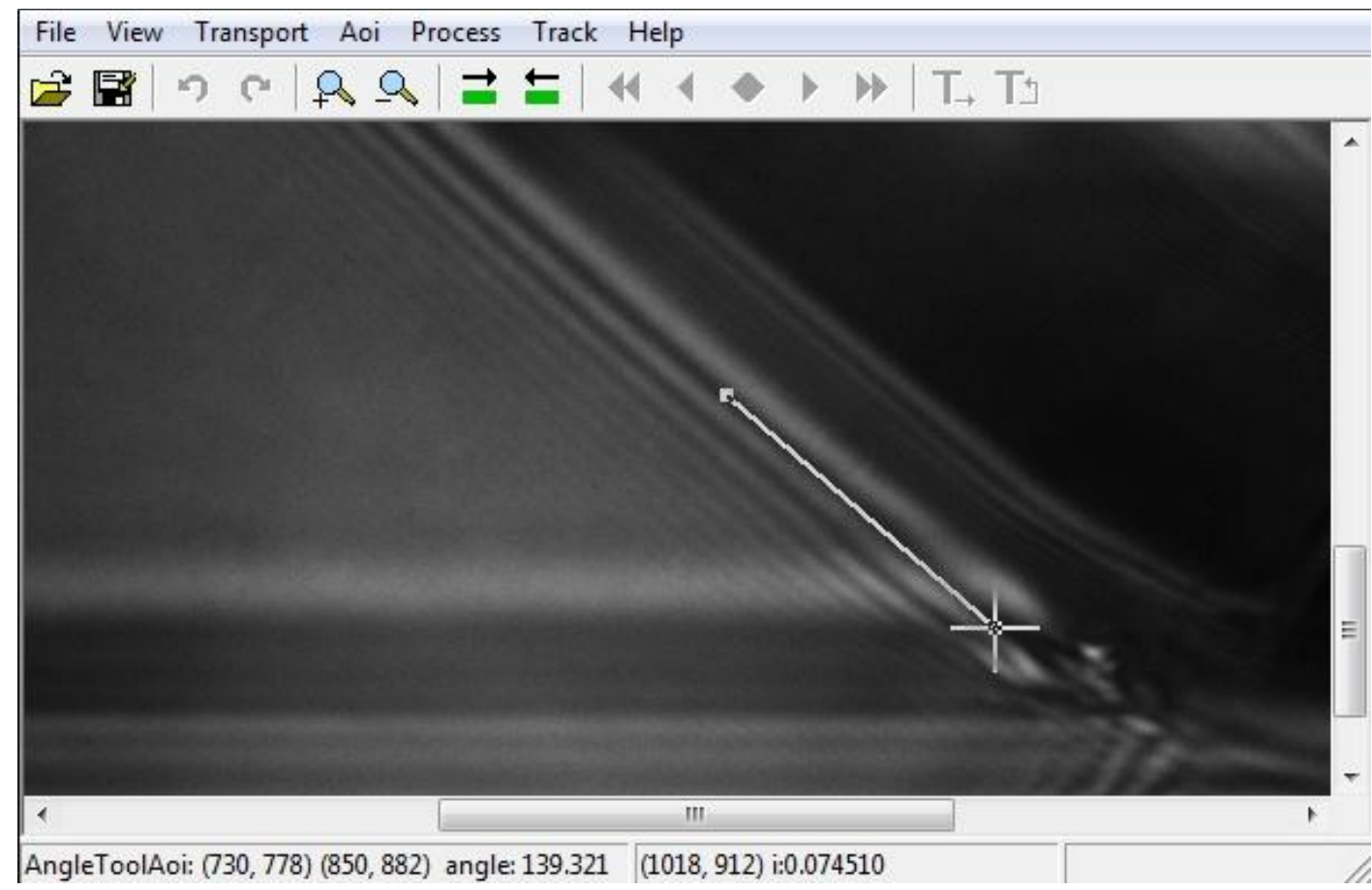

Figure 25 Spotlight is used to measure the angle of the backlit interface. 


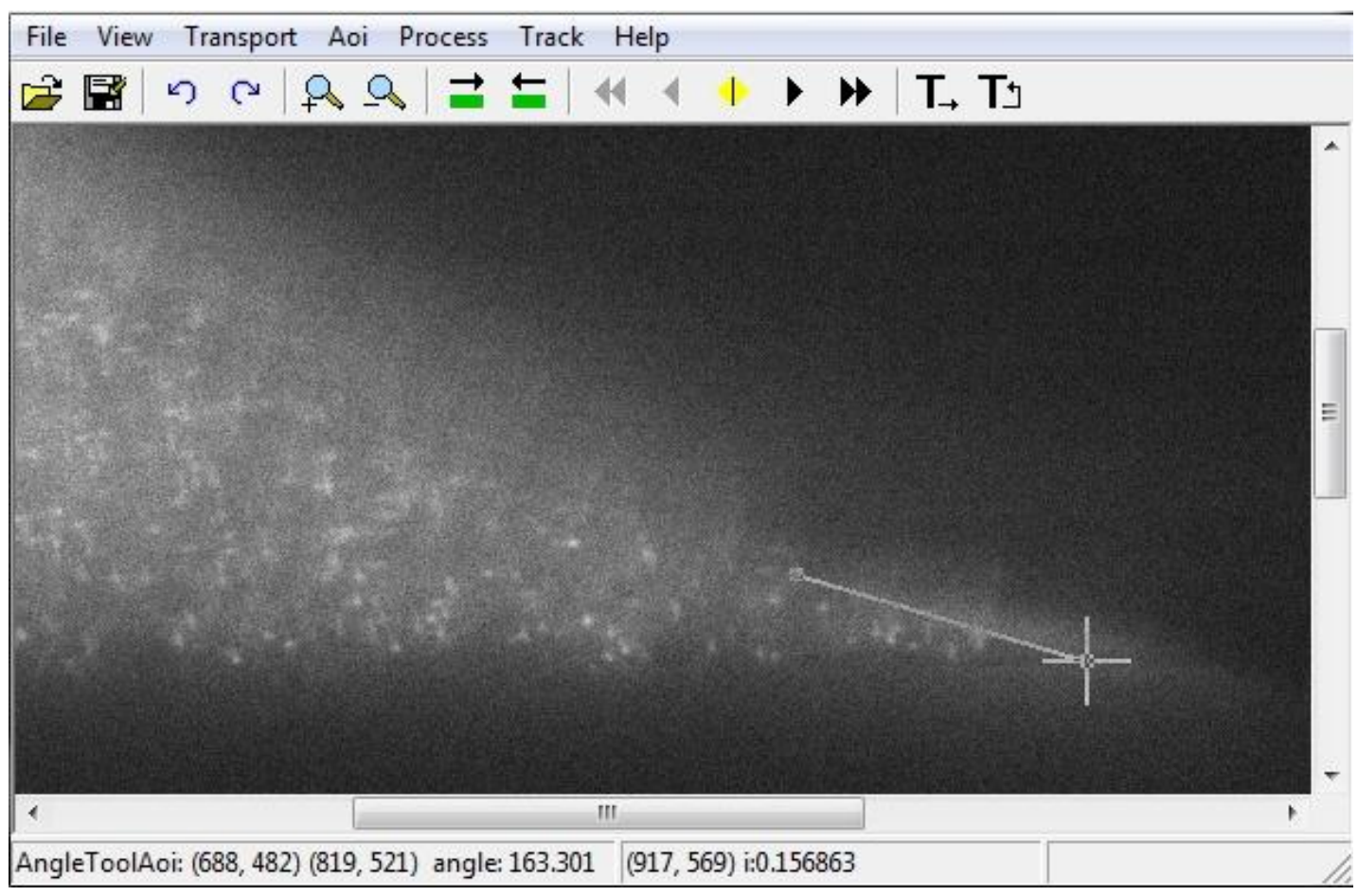

Figure 26 Spotlight is used to measure the angle between fluorescing particles near the interface.

A MATLAB code (see Appendix) was developed to calculate the angle of each velocity vector and display a contour plot. The color gradient allows a visual interrogation of local angles. Probing the plot provides quantitative measurement of apparent contact angles less than $1 \mu \mathrm{m}$ from the contact line. The ultimate accuracy depends on the validity of the velocity vector field. 


\subsection{Error Calculation}

\subsubsection{Velocity Vector Field}

After calculating the velocity data using MATLAB and removing the vectors outside the liquid region with CleanVec, the data is imported into MATLAB for statistical analysis. The MATLAB code (see Appendix) was adapted from [Westerweel 2005]. The code converts the vector data into two matrices, $U$ and V. By comparing each velocity vector to the median of the surrounding vectors, spurious vectors are detected. Spurious vectors are outside the $90^{\text {th }}$ percentile with respect to the median. The MATLAB code outputs the percentage of valid vectors and plots the vector field. Valid vectors are black and spurious vectors are red. This plot allows the quality of the velocity field to be quantitatively determined.

\subsubsection{Contact Angle}

The measured dynamic contact angle depends on the measurement location from the moving contact line. The manual method measures the contact angle at discrete distances from the moving contact line between $1 \mu \mathrm{m}$ and $120 \mu \mathrm{m}$ within \pm $2^{\circ}$. However, the results for a single flow fluctuate as much as $\pm 8^{\circ}$ between images. Contact angle measurements are compared between images collected from a single experiment and between data collected from experiments with the same flow conditions. The average contact angle is reported at a mean distance from the 
contact line for all collected data within one standard deviation. This average dynamic contact angle provides an approximation used to compare flow behavior.

\subsection{Range of Measurement}

Using the methods described above, experiments have been conducted for glycerol and GFS in $30 \mu \mathrm{m} \times 300 \mu \mathrm{m}, 50 \mu \mathrm{m} \times 500 \mu \mathrm{m}$, and $100 \mu \mathrm{m} \times 1000 \mu \mathrm{m}$ glass microchannels. Interface velocities have been measured between $1.7 \mu \mathrm{m} / \mathrm{s}$ and 33 $\mu \mathrm{m} / \mathrm{s}$. Dynamic contact angles have been measured manually between $1.1 \mu \mathrm{m}$ and $120 \mu \mathrm{m}$ from the contact line, and calculated from $\mu$ PIV less than $1 \mu \mathrm{m}$ from the contact line. Average dynamic contact angles range from $12.2^{\circ} \pm 3.7^{\circ}$ to $40.2^{\circ} \pm 2.7^{\circ}$. Capillary numbers ranged from $1.7 \times 10^{-4}$ to $6.2 \times 10^{-4}$ with Reynolds numbers between $8.7 \times 10^{-8}$ and $5.7 \times 10^{-6}$. Bond numbers are $\sim 10^{-3}$. 


\section{Results \& Discussion}

Nearly 100 experiments were conducted to develop the technique to measure interface position, dynamic contact angle, and flow velocities near the moving contact line using $\mu$ PIV. Representative results are presented and discussed below.

\subsection{Interface Position}

Figure 27 shows the relative position of a moving GFS/air interface recorded at 14 intervals along the length of a $100 \mu \mathrm{m} \times 1000 \mu \mathrm{m}$ glass microchannel. With an approximate GFS dynamic viscosity of $5 \mathrm{~Pa} \cdot \mathrm{s}$ and a surface tension of approximately $0.05 \mathrm{~N} / \mathrm{m}$, the interface moved at an average velocity of $2.5 \mu \mathrm{m} / \mathrm{s}$ (estimated from the slope of the linear fit, $R^{2}=0.9972$, shown in Figure 27 ). While a linear fit is an accurate approximation, Figure 28 shows that the interface motion fits the theoretical model of $U \sim t^{1 / 2}$ with an $R^{2}=0.9998$. The Capillary number was $\mathrm{Ca} \approx$ $2.5 \times 10^{-4}$ 


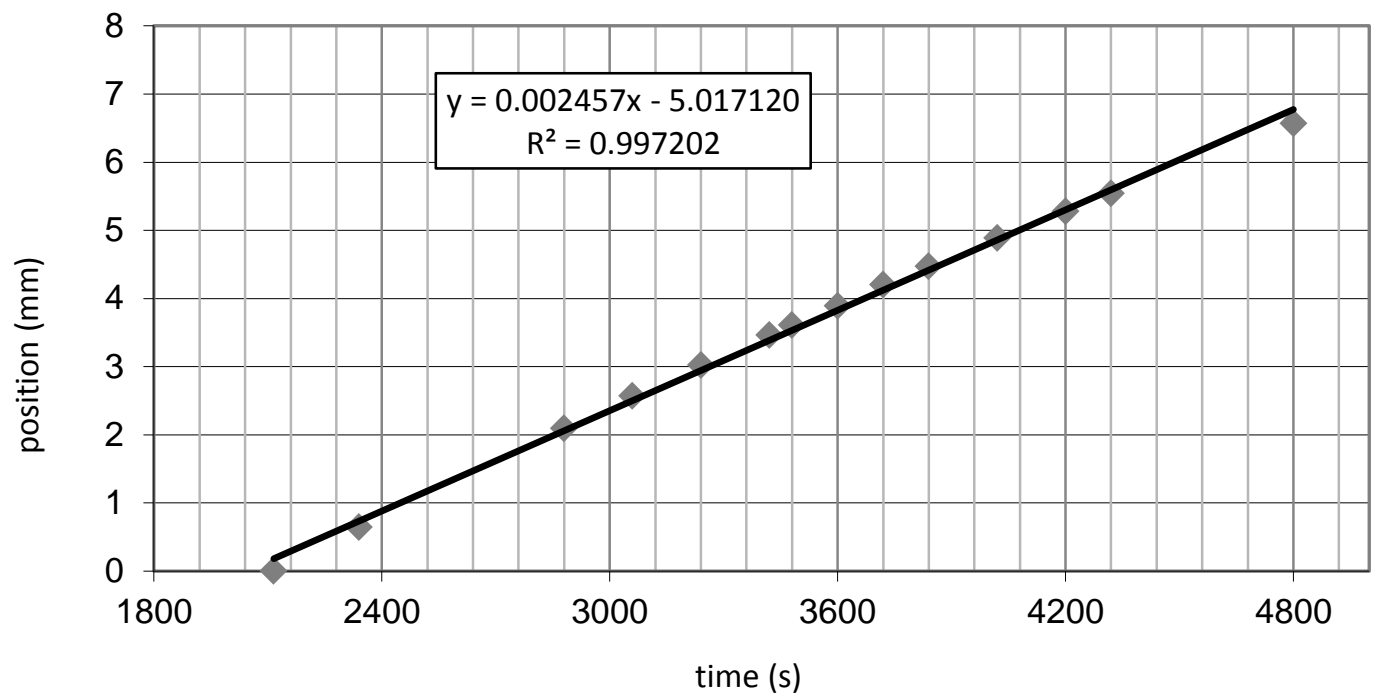

Figure 27 The interface position of GFS in a $100 \mu \mathrm{m} \times 1000 \mu \mathrm{m}$ microchannel with a linear fit approximation.

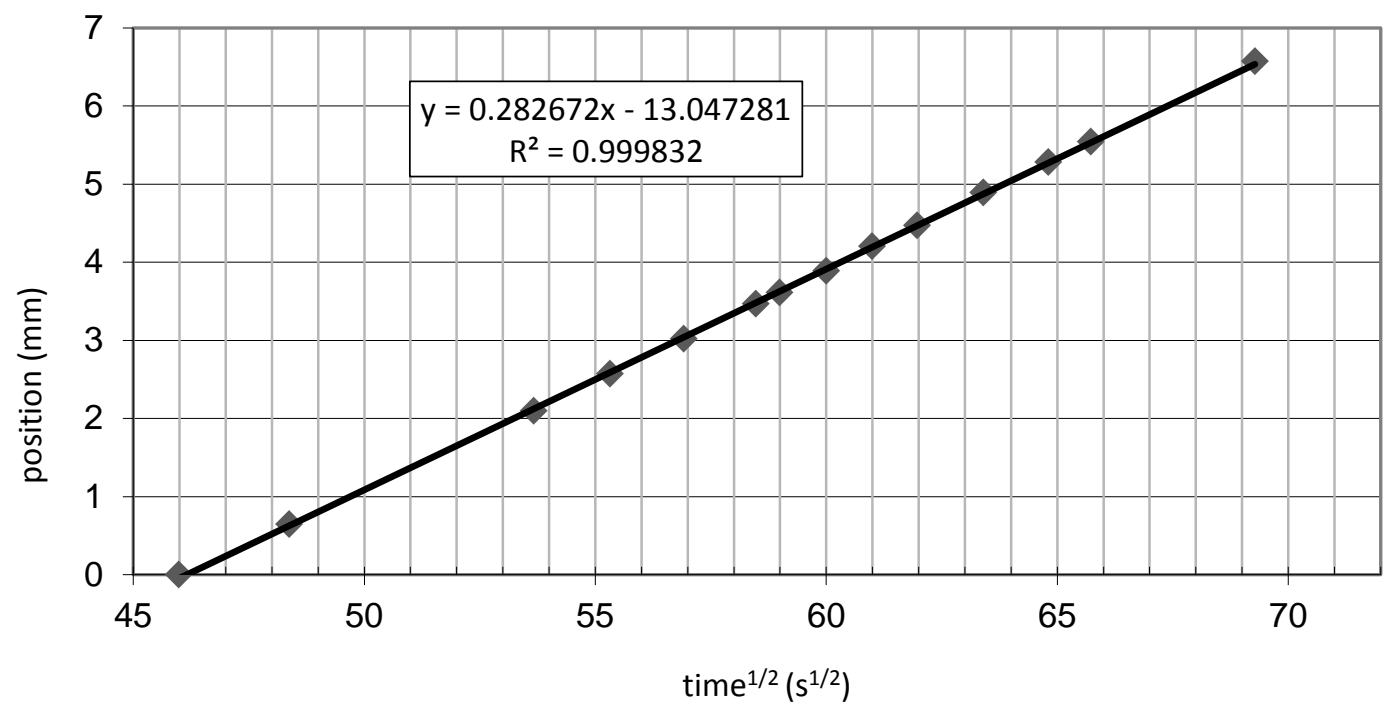

Figure 28 The interface position of GFS in a $100 \mu \mathrm{m} \times 1000 \mu \mathrm{m}$ microchannel showing the predicted $\mathrm{t}^{1 / 2}$ behavior. 
Figure 29 shows the position of a moving glycerol/air interface recorded at 12 intervals along the length of a $50 \mu \mathrm{m} \times 500 \mu \mathrm{m}$ microchannel. The approximate dynamic viscosity of glycerol is $1.2 \mathrm{~Pa} \cdot \mathrm{s}$ and the approximate surface tension is 0.06 $\mathrm{N} / \mathrm{m}$. The glycerol/air interface moved at an average velocity of $14.8 \mu \mathrm{m} / \mathrm{s}$. A linear fit correlated the data well with an $\mathrm{R}^{2}=0.9951$. Figure 30 shows that the interface motion fits the theoretical model of $U \sim t^{1 / 2}$ with an $R^{2}=0.9997$. The Capillary number was $\mathrm{Ca} \approx 2.8 \times 10^{-4}$.

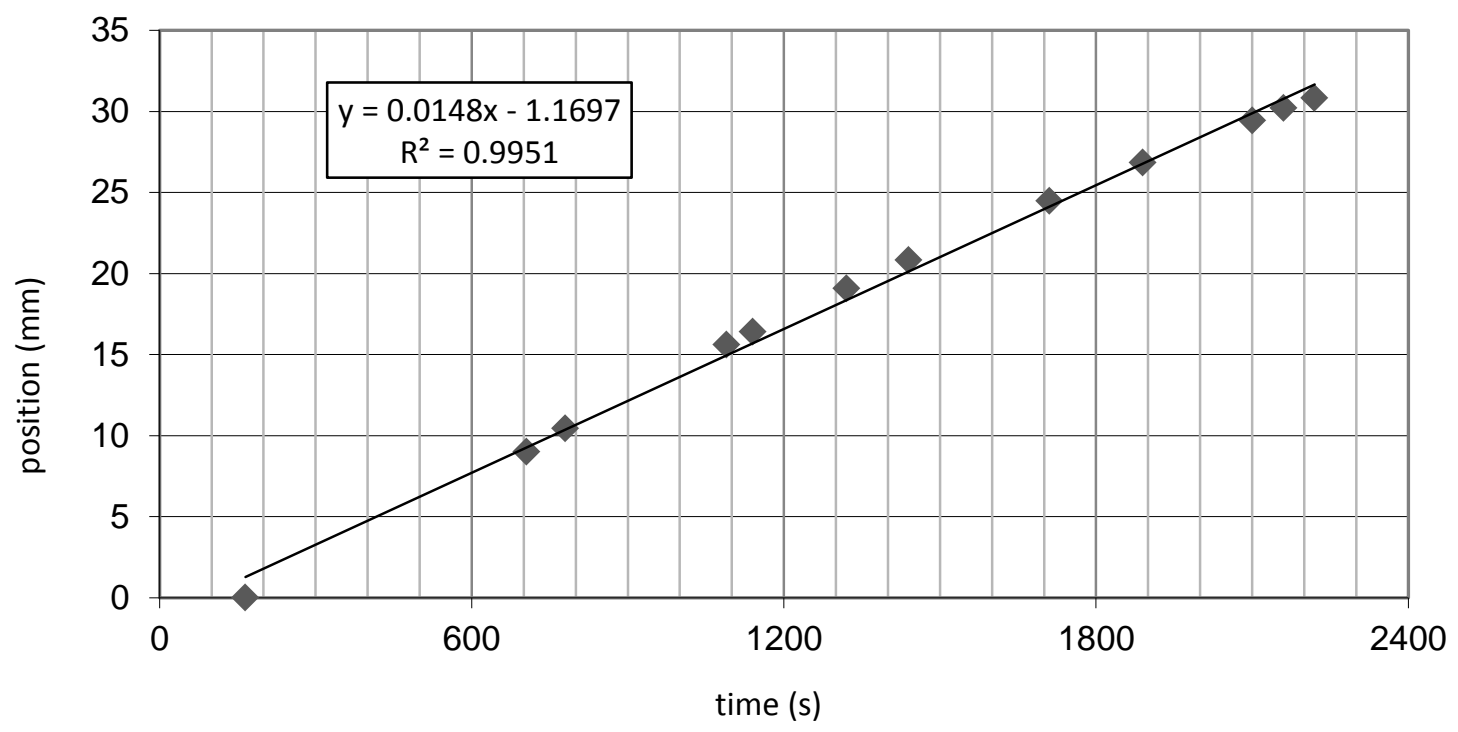

Figure 29 The interface position of glycerol in a $50 \mu \mathrm{m}$ × $500 \mu \mathrm{m}$ microchannel with a linear fit approximation. 


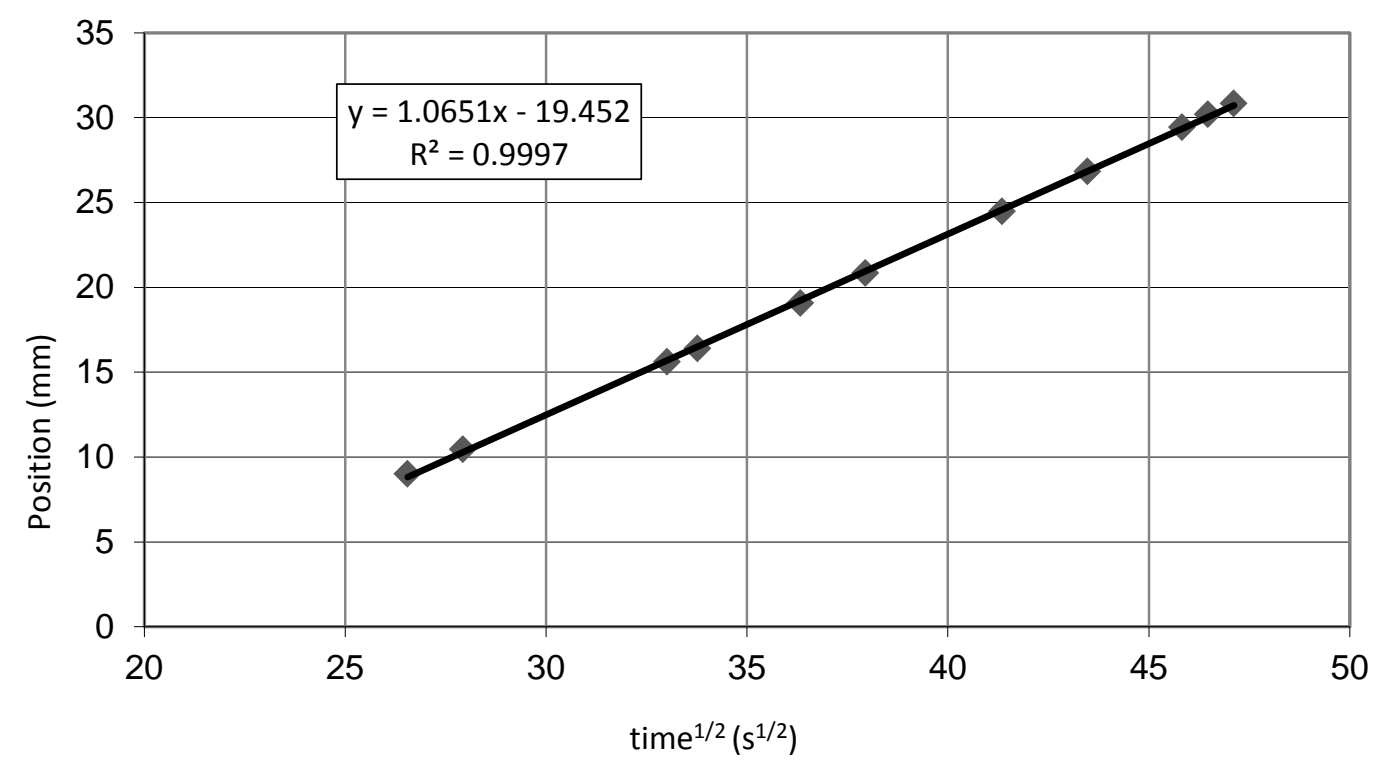

Figure 30 The interface position of glycerol in a $50 \mu \mathrm{m} \times 500 \mu \mathrm{m}$ microchannel showing the predicted $\mathrm{t}^{1 / 2}$ behavior.

With the approximately linear motion of the interface, a linear stage motion accurately follows the interface for short durations. This allows a significant number of images to be captured without interface movement for the measurement of dynamic contact angles and the calculation of local fluid velocities. 


\subsection{Contact Angle}

Figure 31 shows the manual measurement of dynamic contact angles for glycerol moving in a $50 \mu \mathrm{m} \times 500 \mu \mathrm{m}$ microchannel. For eight experiments with a median Capillary number of approximately $\mathrm{Ca} \approx 2.85 \times 10^{-4}$, the average contact angle measured at a mean distance of $26 \mu \mathrm{m}$ from the leading edge was $18.9^{\circ} \pm 7.19^{\circ}$.

Figure 32 shows the manual measurement of dynamic contact angles for GFS moving in a $100 \mu \mathrm{m} \times 1000 \mu \mathrm{m}$ channel. With a median Capillary number of $\mathrm{Ca} \approx$ $2.60 \times 10^{-4}$, the average contact angle measured at a mean distance of $36 \mu \mathrm{m}$ from the leading edge was $36.1^{\circ} \pm 7.79^{\circ}$.

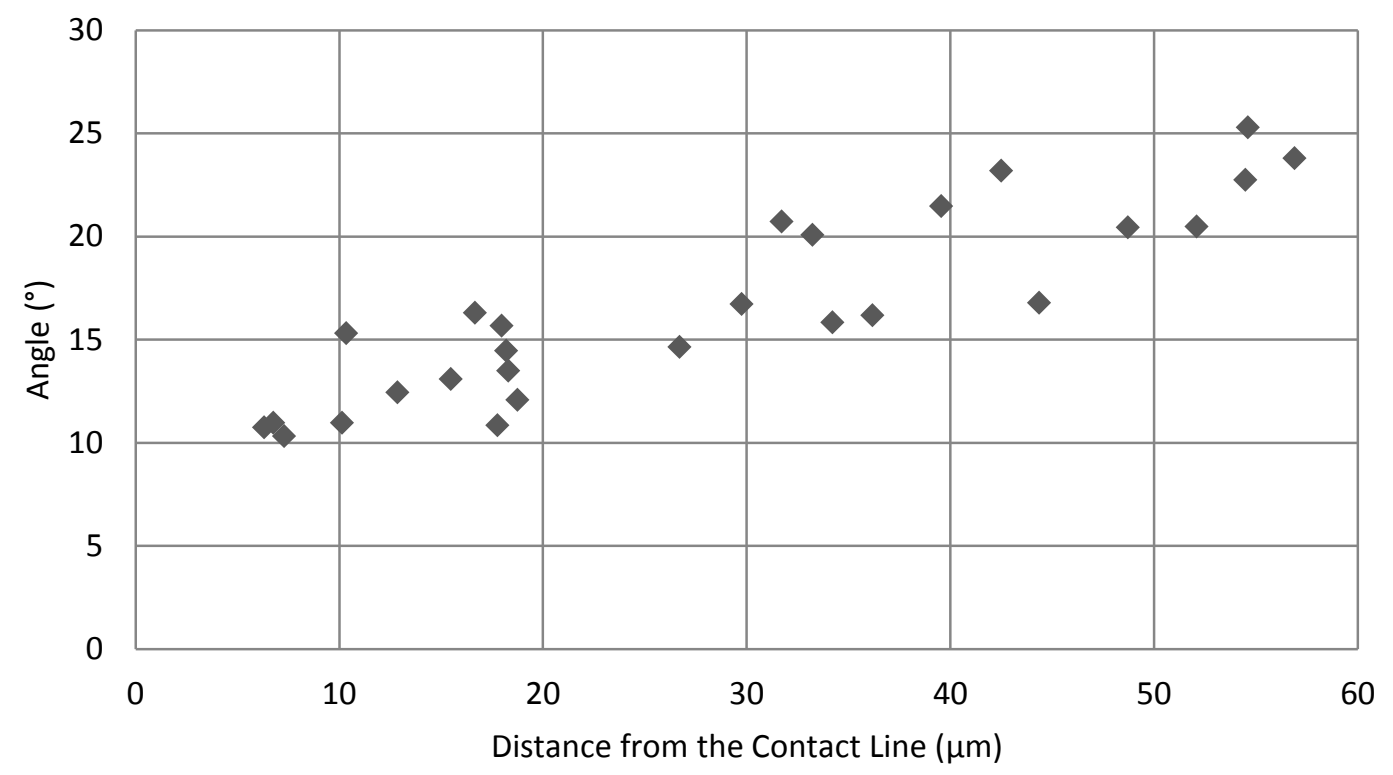

Figure 31 Manual contact angle measurement for glycerol in a $50 \mu \mathrm{m} \times 500 \mu \mathrm{m}$ channel using Spotlight. 


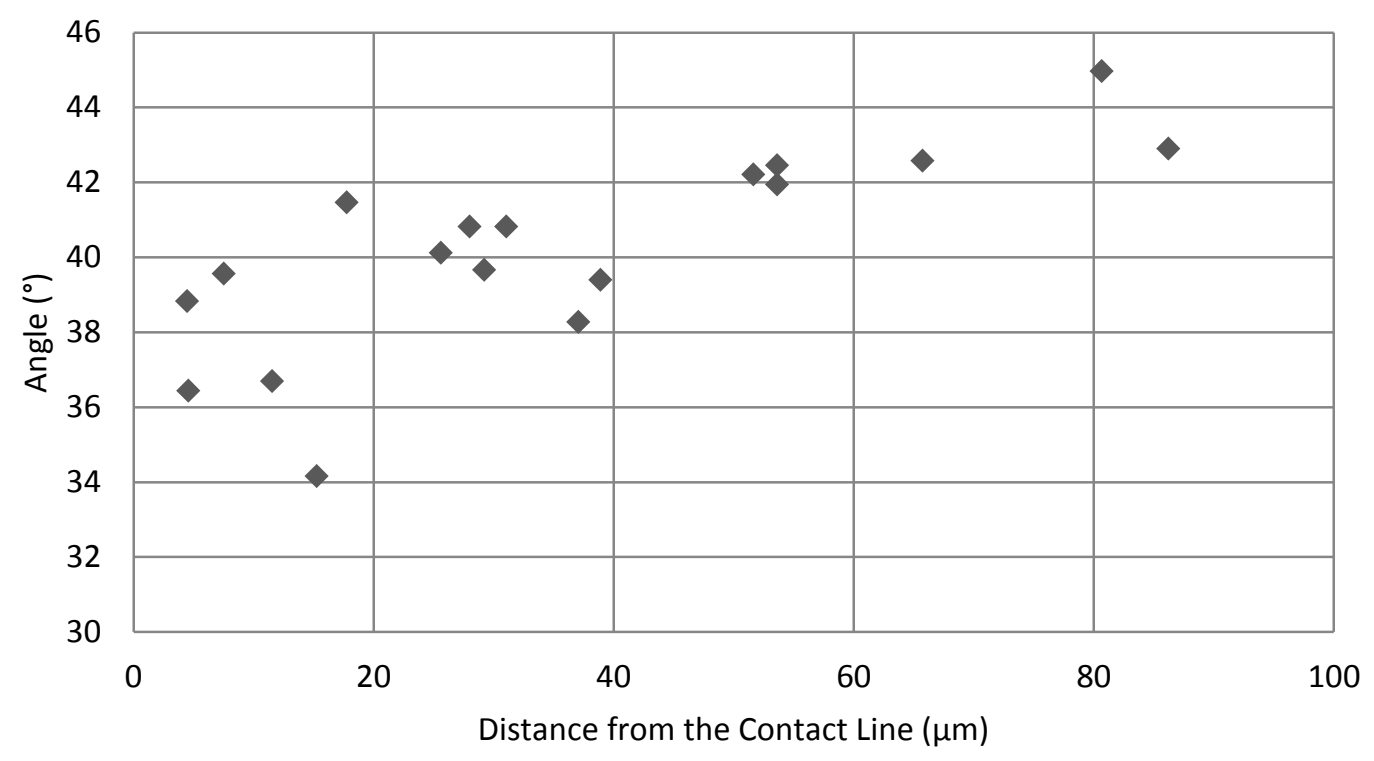

Figure 32 Manual contact angle measurement for the glucose/fructose solution (GFS) in a $100 \mu \mathrm{m} x$ $1000 \mu \mathrm{m}$ channel using Spotlight.

While Figure 31 and Figure 32 seem to indicate significant variation in the measurement of a single dynamic contact angle, the trend towards higher angles away from the contact line caused by meniscus curvature confirms that angle measurements in this region are geometry dependent and therefore not at a high enough resolution to explore the geometry-independent inner region.

Table 2 shows the results of 14 contact angle experiments with manual measurements of the dynamic contact angle for glycerol and GFS in $30 \mu \mathrm{m} \times 300 \mu \mathrm{m}$, $50 \mu \mathrm{m} \times 500 \mu \mathrm{m}$, and $100 \mu \mathrm{m} \times 1000 \mu \mathrm{m}$ microchannels. Measurements show a standard deviation of $4.91^{\circ}$. 
Table 2 Manually measured dynamic contact angles.

\begin{tabular}{|c|c|c|c|c|c|c|c|}
\hline \multirow{2}{*}{ Fluid } & \multirow{2}{*}{$\begin{array}{l}\text { Channel } \\
\text { Size }(\mu \mathrm{m})\end{array}$} & \multicolumn{3}{|c|}{ Distance from the Contact Line $(\mu \mathrm{m})$} & \multirow{2}{*}{$\begin{array}{c}\text { Average Contact } \\
\text { Angle }\left({ }^{\circ}\right)\end{array}$} & \multirow{2}{*}{$\pm\left({ }^{\circ}\right)$} & \multirow{2}{*}{$\begin{array}{l}\mathrm{Ca} * \\
10^{-4}\end{array}$} \\
\hline & & Minimum & Maximum & Median & & & \\
\hline \multirow{12}{*}{$\begin{array}{l}\overline{0} \\
\frac{0}{凶} \\
\stackrel{ত}{ত}\end{array}$} & \multirow{3}{*}{$30 \times 300$} & 9.4 & 54 & 17 & 14.6 & 7.0 & 1.9 \\
\hline & & 7.5 & 65 & 34 & 14.9 & 3.1 & 2.3 \\
\hline & & 3.8 & 58 & 19 & 12.2 & 3.7 & 2.0 \\
\hline & \multirow{8}{*}{$50 \times 500$} & 6.3 & 64 & 32 & 27.1 & 6.4 & 2.0 \\
\hline & & 4.3 & 48 & 23 & 22.5 & 5.8 & 2.0 \\
\hline & & 4.4 & 52 & 26 & 28.3 & 8.1 & 5.7 \\
\hline & & 18 & 69 & 49 & 20.4 & 5.0 & 6.2 \\
\hline & & 3.0 & 55 & 22 & 18.1 & 5.9 & 2.9 \\
\hline & & 2.7 & 50 & 19 & 20.8 & 7.0 & 2.8 \\
\hline & & 1.1 & 35 & 15 & 24.5 & 7.8 & 3.5 \\
\hline & & 6.3 & 57 & 27 & 16.5 & 4.5 & 2.6 \\
\hline & $100 \times 1000$ & 10 & 79 & 46 & 16.1 & 2.0 & 5.6 \\
\hline \multirow{2}{*}{ 茨 } & \multirow{2}{*}{$100 \times 1000$} & 2.0 & 69 & 42 & 23.9 & 4.0 & 2.5 \\
\hline & & 4.5 & 86 & 30 & 40.2 & 2.7 & 1.7 \\
\hline
\end{tabular}

The measurement of dynamic contact angles using the manual angle tool in Spotlight provides an approximate angle near a moving interface and a quantification of meniscus curvature. However, manual measurements cannot accurately measure apparent contact angles in the inner region. Figure 33 shows, as a contour plot, the angle of the velocity vectors measured with $\mu$ PIV for GFS in a $100 \mu \mathrm{m} \times 1000 \mu \mathrm{m}$ channel moving at $1.7 \mu \mathrm{m} / \mathrm{s}\left(\mathrm{Ca}=1.7 \times 10^{-4}\right)$. The contact angle is consistent in the field of view at about $40^{\circ}$ and shows agreement between the manual measurement and the angle of the velocity vectors near the interface. 


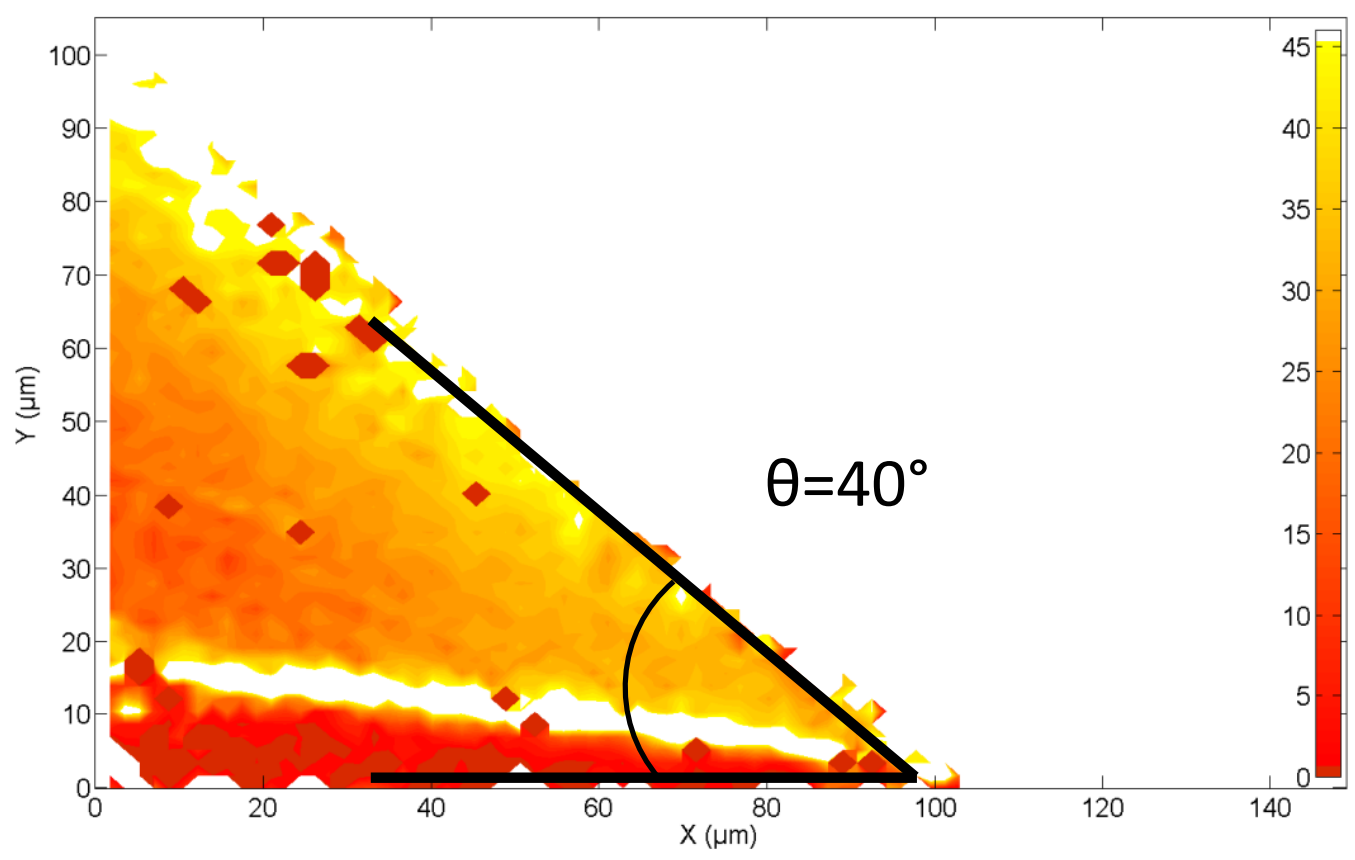

Figure 33 Dynamic contact angle measurement using $\mu$ PIV for GFS in a $100 \mu \mathrm{m} \times 1000 \mu \mathrm{m}$ channel.

Figure 34 shows the vector angles calculated for glycerol flowing in a $30 \mu \mathrm{m} \times 300$ $\mu \mathrm{m}$ channel at $10.5 \mu \mathrm{m} / \mathrm{s}$ with $\mathrm{Ca}=1.9 \times 10^{-4}$. The contact angle is $27^{\circ}$ at $100 \mu \mathrm{m}$ from the contact line, $13^{\circ}$ at $60 \mu \mathrm{m}$ from the contact line, and $5^{\circ}$ at $10 \mu \mathrm{m}$ from the contact line. Angle measurements in the geometrically dependent region away from the leading edge agree with manual measurement and demonstrate the importance to measure contact angles less than $10 \mu \mathrm{m}$ from the contact line to explore the region where previous experimental studies have deviated from theoretical prediction. 


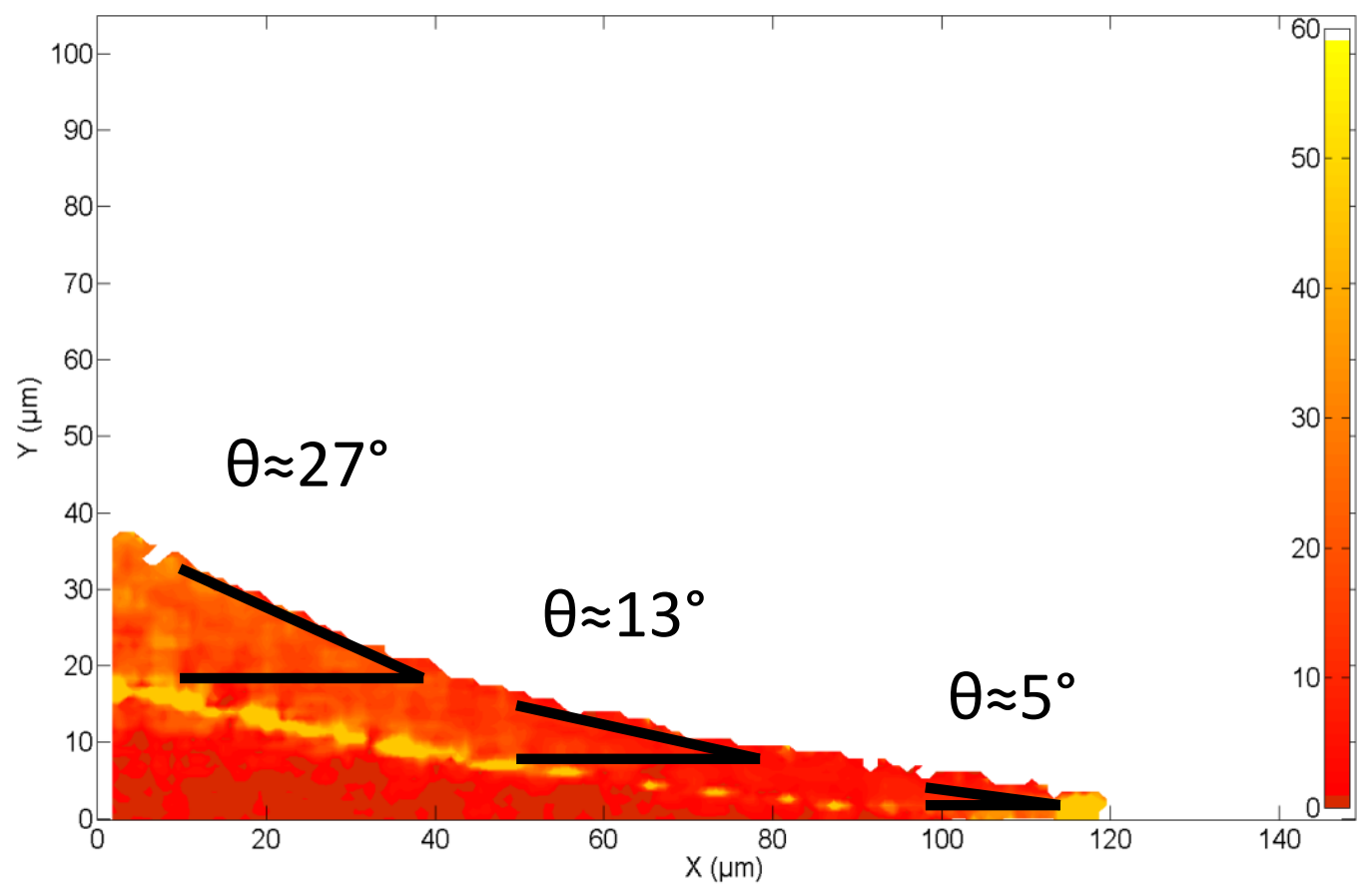

Figure 34 Dynamic contact angle measurements using $\mu$ PIV at varying distance from the interface for glycerol in a $30 \mu \mathrm{m} \times 300 \mu \mathrm{m}$ channel.

High resolution contact angle values are observed less than $1 \mu \mathrm{m}$ from the contact line in Figure 35. While the apparent dynamic contact angle $30 \mu \mathrm{m}$ from the contact line is approximately $8.5^{\circ}$, the exact values of vector angles close to the liquid/gas/solid intersection are $55^{\circ}$ or higher. It is possible that seed particles may be too large to reach the tip of the liquid/gas interface at the solid wall. However, the deviation in measured angle from the geometrically dependent meniscus shape shows promise that the vector angle measurement technique may be used to measure angles within the intermediate and inner regions. 


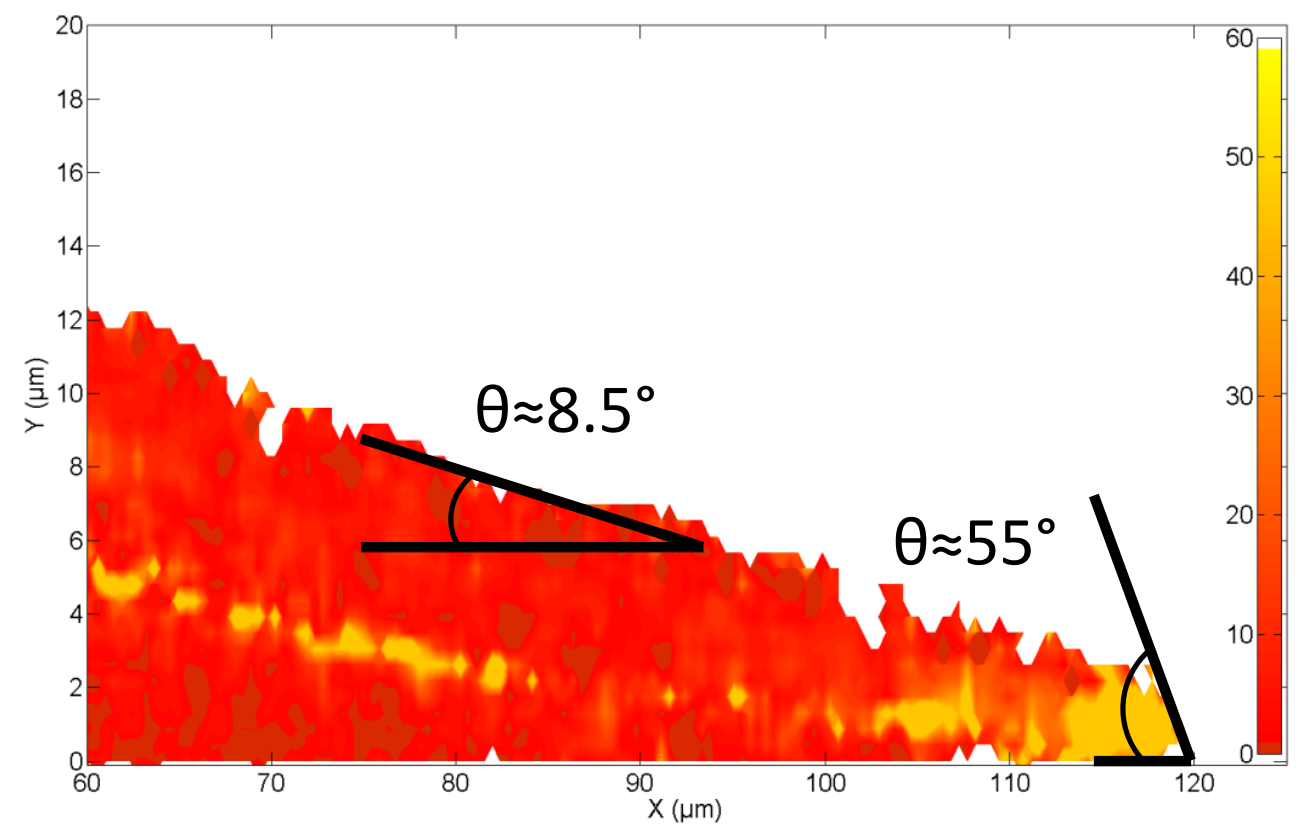

Figure 35 Dynamic contact angle measurements near the interface using $\mu$ PIV data. While apparent angle is small, the angle increases drastically within $5 \mu \mathrm{m}$ of the contact line.

\subsection{Fluid Velocities 1 micron from the Moving Contact Line}

Figure 36 shows the velocity field near the moving contact line for glycerol in a 50 $\mu \mathrm{m} \times 500 \mu \mathrm{m}$ microchannel with a Capillary number of $\mathrm{Ca}=5.7 \times 10^{-4} .45 \mu \mathrm{PIV}$ image pairs were analyzed using a 48 pixel interrogation region with a $75 \%$ overlap (a resolution of $5.232 \mu \mathrm{m}$ and a vector spacing of $1.308 \mu \mathrm{m})$. Applying the median test, the data is of low quality with only $68.67 \%$ of the vectors being valid. Figure 37 shows an increased resolution of $3.488 \mu \mathrm{m}$ by reducing the interrogation region to 32 pixels. 
This results in a reduction of valid vectors to $48.95 \%$. Spurious vectors appear randomly scattered throughout the vector field and are likely due to noisy images or too few image pairs.

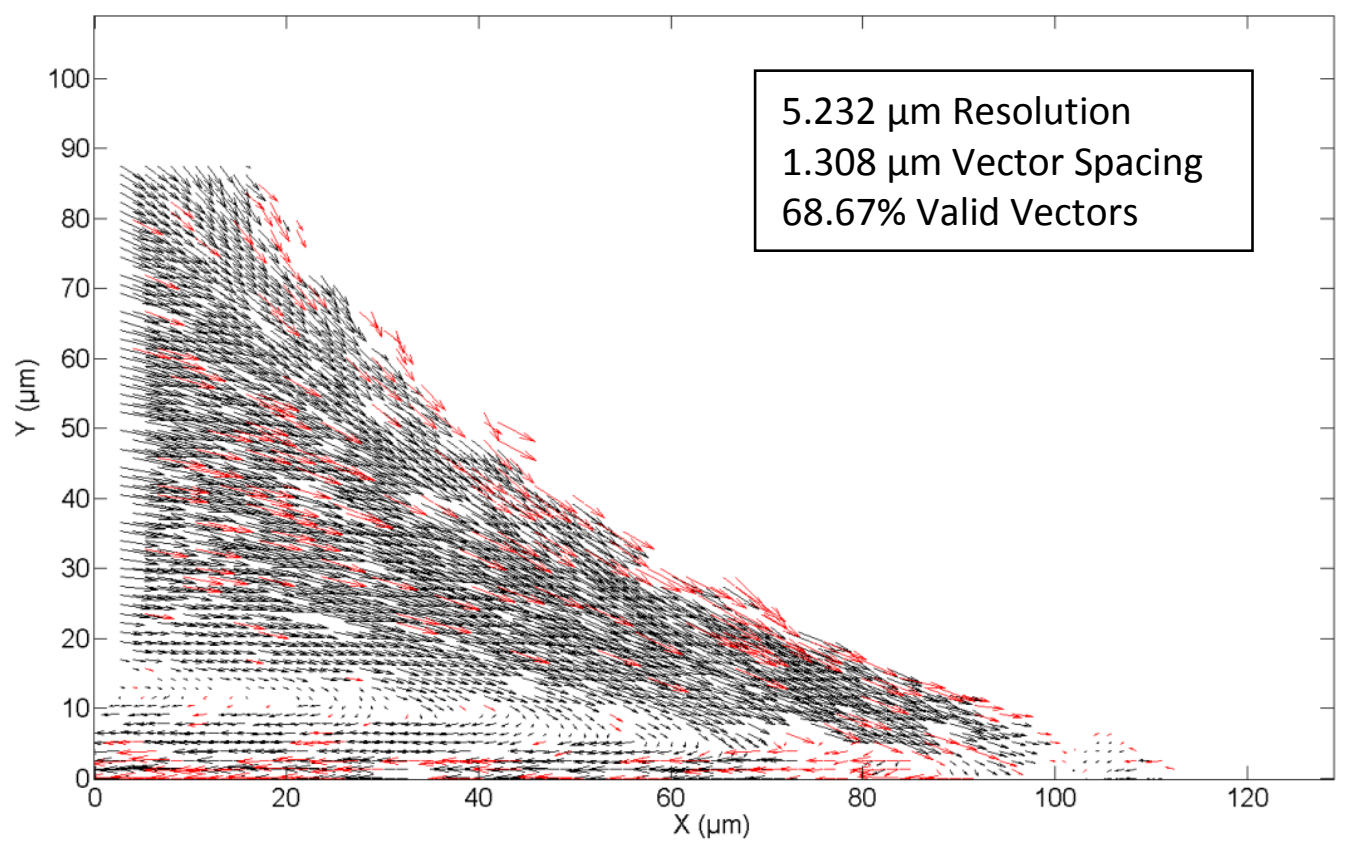

Figure $36 \mu \mathrm{PIV}$ velocity vectors for glycerol in a $50 \mu \mathrm{m} \times 500 \mu \mathrm{m}$ microchannel with $\mathrm{Ca}=5.7 \mathrm{E}-4.45$ pairs of images were analyzed using a 48 pixel interrogation region with a $75 \%$ overlap. 


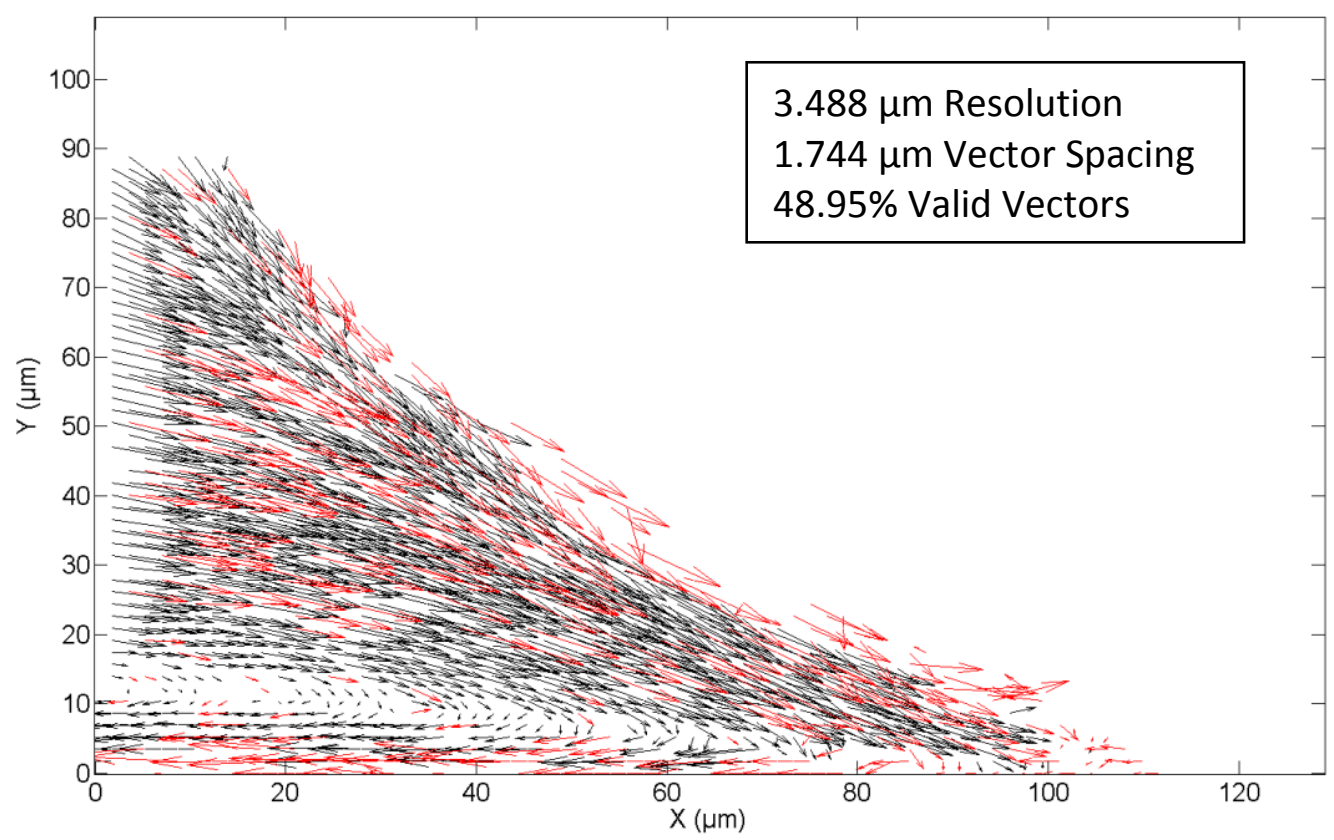

Figure $37 \mu \mathrm{PIV}$ velocity vectors for glycerol in a $50 \mu \mathrm{m} \times 500 \mu \mathrm{m}$ microchannel with $\mathrm{Ca}=5.7 \mathrm{E}-4.45$ pairs of images were analyzed using a 32 pixel interrogation region with a $50 \%$ overlap.

Measuring velocities of GFS in a $100 \mu \mathrm{m} \times 1000 \mu \mathrm{m}$ channel produced a higher number of valid vectors. 224 pairs of images of the flow were captured. Figure 38 shows the velocity field for GFS in a $100 \mu \mathrm{m}$ x $1000 \mu \mathrm{m}$ channel. The average velocity of the interface was $1.7 \mu \mathrm{m} / \mathrm{s}\left(\mathrm{Ca}=1.7 \times 10^{-4}\right)$. With 224 image pairs analyzed using a 32 pixel interrogation region with a $50 \%$ overlap (a velocity resolution of $3.488 \mu \mathrm{m}$ and vector spacing of $1.744 \mu \mathrm{m}$ ) the median test showed $91.10 \%$ of the vectors are valid. This is a significant increase (49\% to 91\%) from 45 image pairs. 


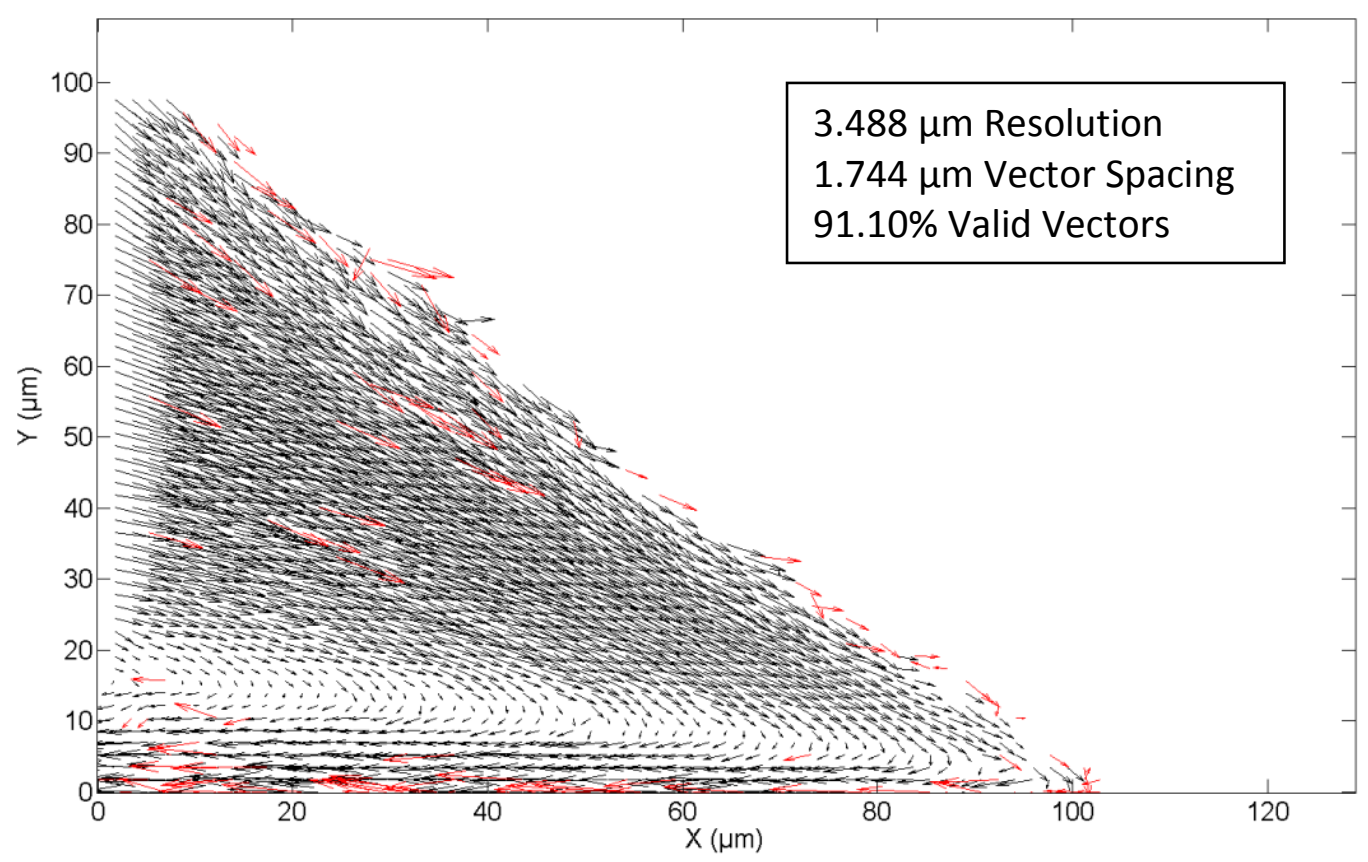

Figure $38 \mu$ PIV velocity vectors for GFS in a $100 \mu \mathrm{m} \times 1000 \mu \mathrm{m}$ microchannel with $\mathrm{Ca}=1.7 \mathrm{E}-4.224$ pairs of images were analyzed using a 32 pixel interrogation region with a 50\% overlap.

Figure 39 shows the velocity field for the same flow at a higher resolution ( 24 pixel interrogation region with a $75 \%$ overlap). The results show a reduction in overall vector quality to $87.26 \%$. It is important to note the location of the spurious vectors. Errors occur near the wall, along the interface, and away from the contact line. Velocity vectors are valid $10 \mu \mathrm{m}$ from the contact line. 


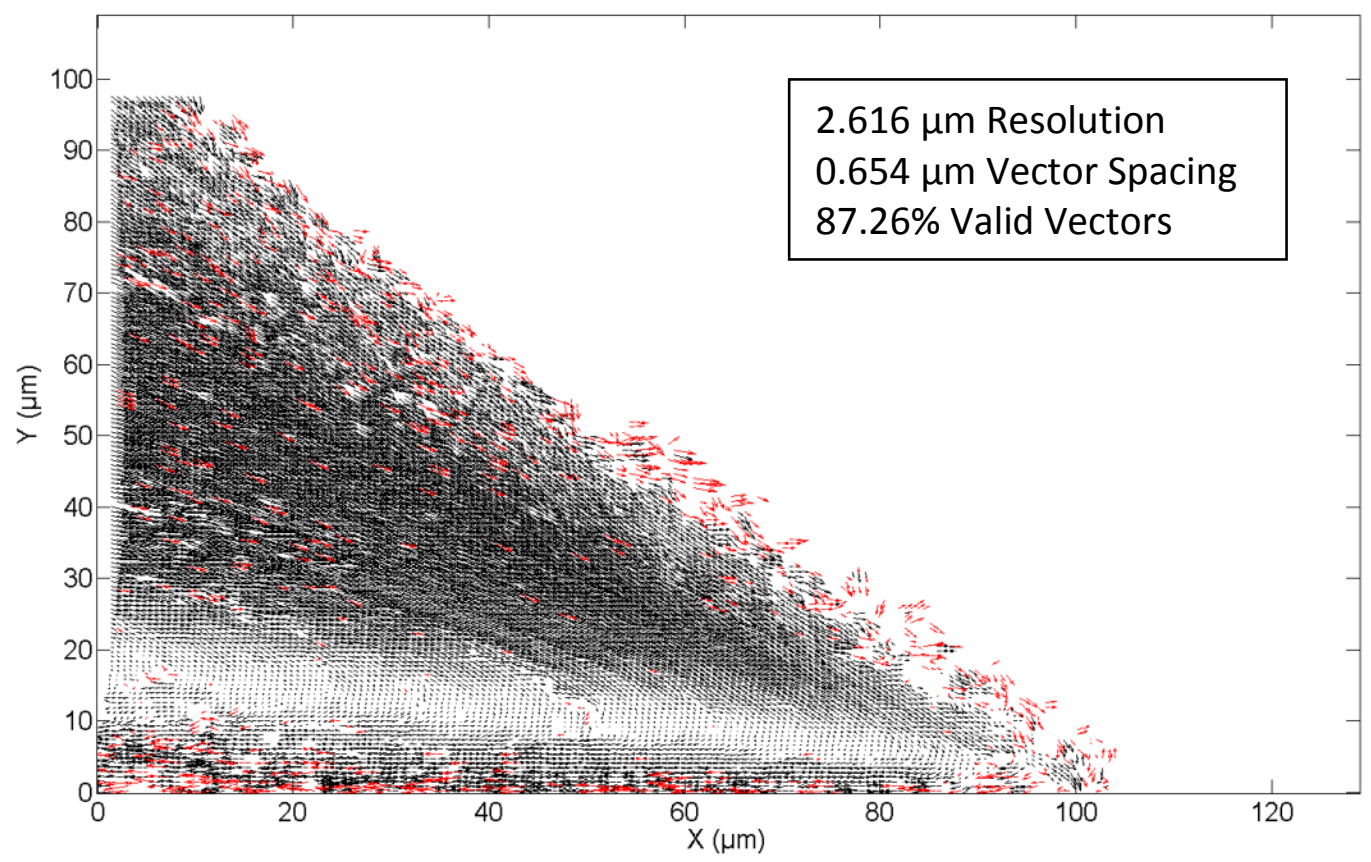

Figure $39 \mu \mathrm{PIV}$ velocity vectors for glycerol in a $50 \mu \mathrm{m} \times 500 \mu \mathrm{m}$ microchannel with $\mathrm{Ca}=1.7 \mathrm{E}-4 . \quad 224$ pairs of images were analyzed using a 24 pixel interrogation region with a $75 \%$ overlap.

Figure 40 is the velocity field of glycerol flowing in a $30 \mu \mathrm{m} \times 300 \mu \mathrm{m}$ channel with an average interface velocity of $10.5 \mu \mathrm{m} / \mathrm{s}\left(\mathrm{Ca}=1.9 \times 10^{-4}\right)$. With 86 images, a low resolution interrogation size of 64 pixels yielded a high percentage of valid vectors; however spurious vectors appear within $40 \mu \mathrm{m}$ of the contact line, most likely a result of velocity gradients at the leading edge. 


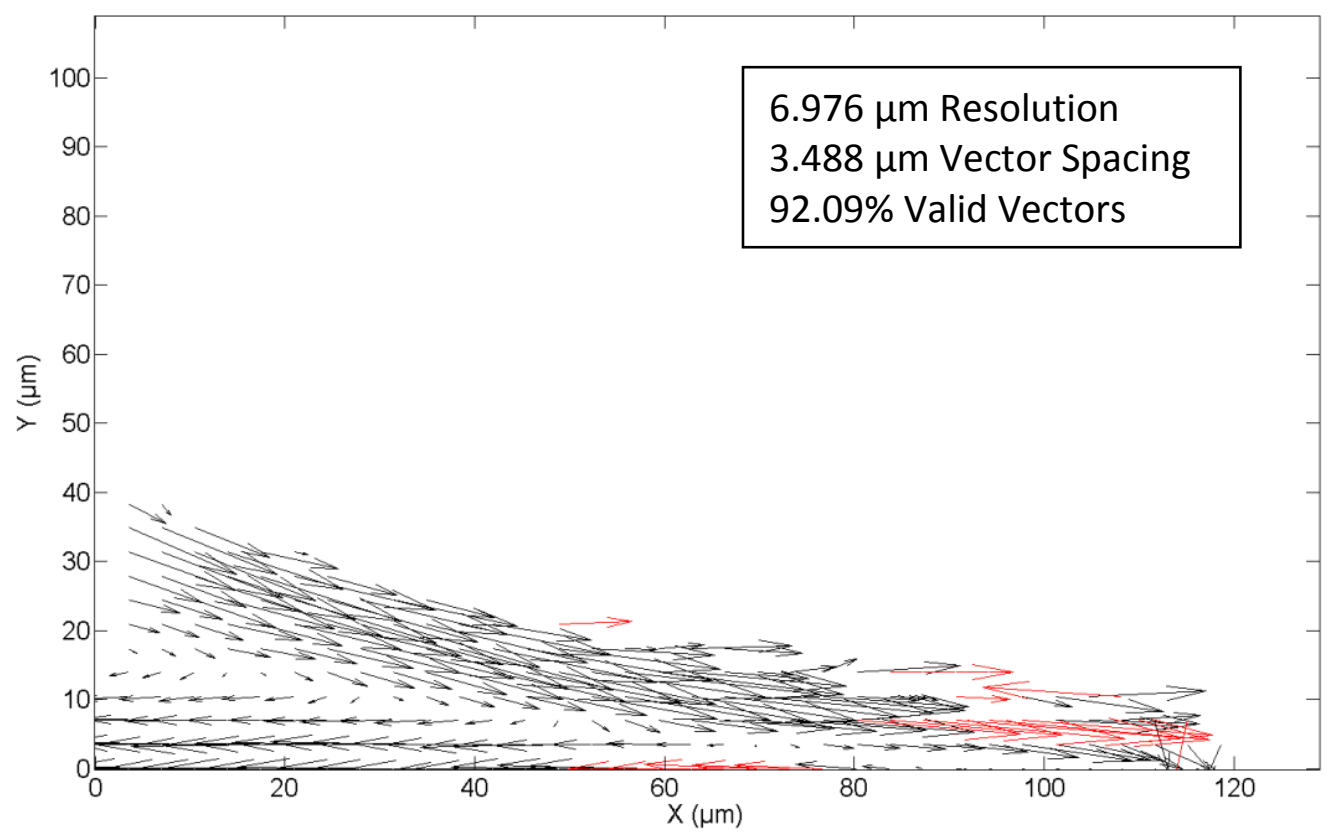

Figure $40 \mu \mathrm{PIV}$ velocity vectors for glycerol in a $30 \mu \mathrm{m}$ x $300 \mu \mathrm{m}$ microchannel with Ca $=1.9 \mathrm{E}-4.43$ pairs of images were analyzed using a 64 pixel interrogation region with a $50 \%$ overlap.

Figure 41 shows that increasing the resolution to $3.488 \mu \mathrm{m}$ using a 32 pixel interrogation region increases the number of valid vectors to $96.82 \%$. This is in contrast to the results previously presented and indicates that while poor image quality will result in a decrease in valid vectors when the resolution is increased, high quality images result in higher quality vector fields. As the interrogation region shrinks to the length scales of the flow patterns, the number of spurious vectors decreases. 


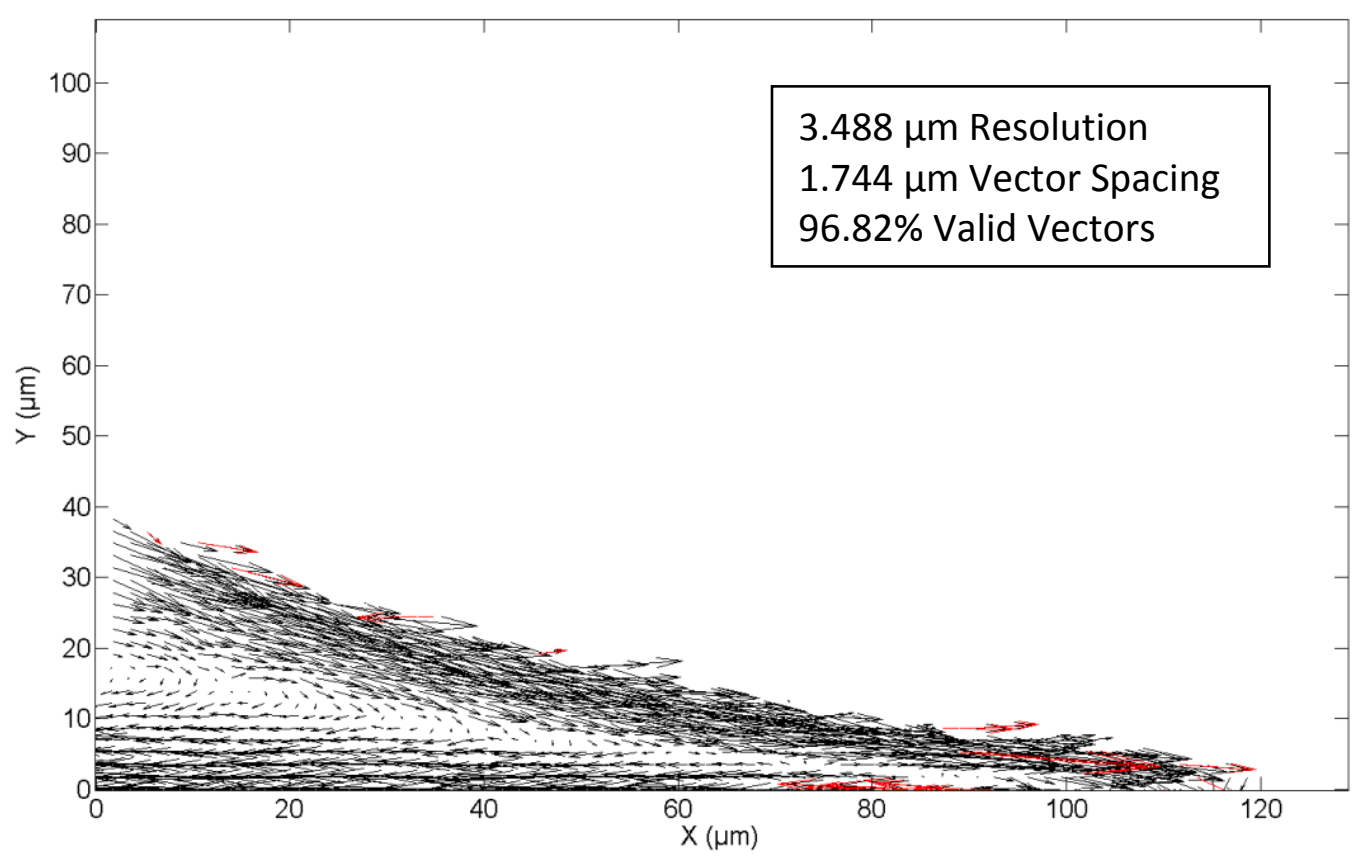

Figure $41 \mu \mathrm{PIV}$ velocity vectors for glycerol in a $30 \mu \mathrm{m}$ x $300 \mu \mathrm{m}$ microchannel with $\mathrm{Ca}=1.9 \mathrm{E}-4.43$ pairs of images were analyzed using a 32 pixel interrogation region with a $50 \%$ overlap.

\subsection{Fluid Velocities $<1$ micron from the Moving Contact Line}

The $\mu$ PIV images of glycerol in a $30 \mu \mathrm{m} \times 300 \mu \mathrm{m}$ channel described above are the highest quality images captured in nearly 100 experiments. This level of quality allows the interrogation region to be greatly reduced. Figure 42 shows the velocity field using a 16 pixel interrogation region with a $50 \%$ overlap. This results in a resolution of $1.744 \mu \mathrm{m}$ with a vector spacing of $872 \mathrm{~nm}$. The median test shows $97.21 \%$ valid vectors. 


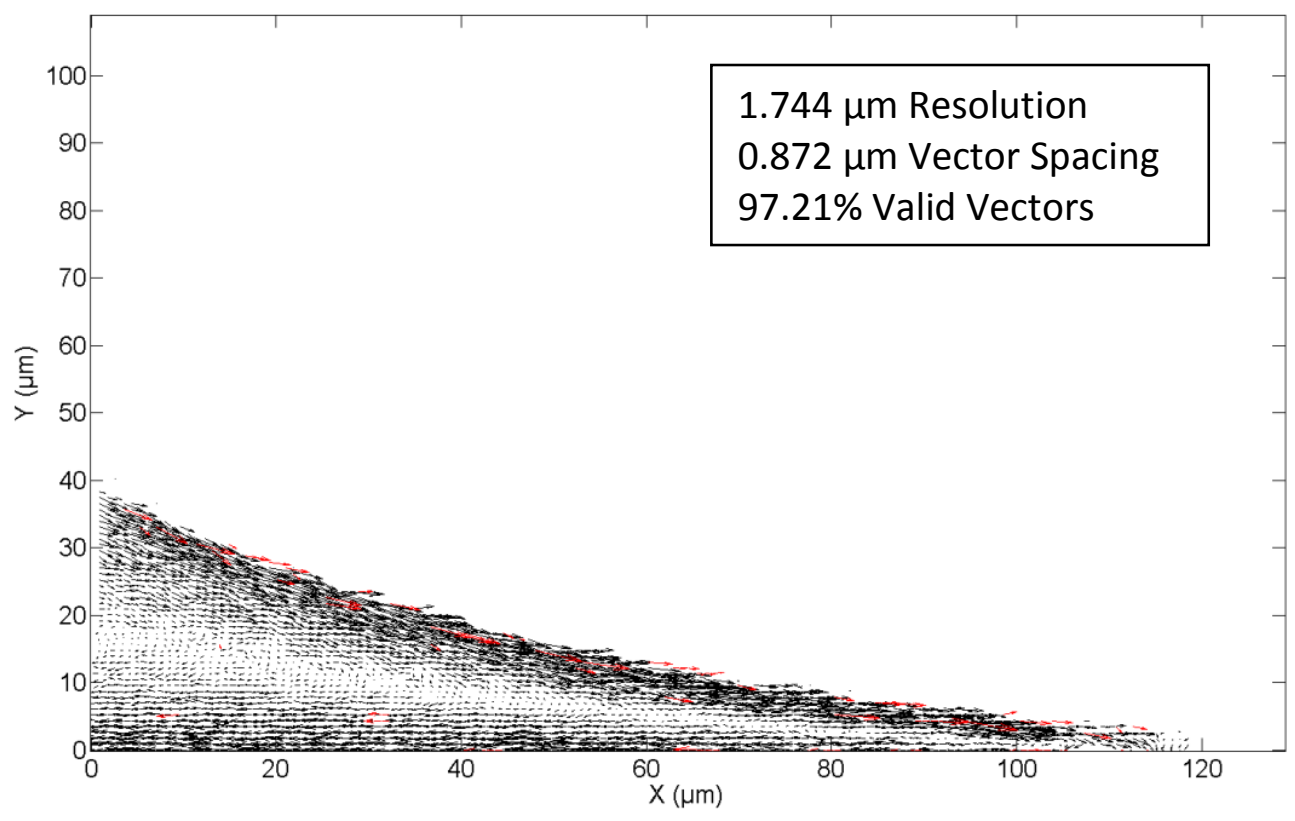

Figure $42 \mu$ PIV velocity vectors for glycerol in a $30 \mu \mathrm{m} \times 300 \mu \mathrm{m}$ microchannel with $\mathrm{Ca}=1.9 \mathrm{E}-4.43$ pairs of images were analyzed using a 16 pixel interrogation region with a $50 \%$ overlap.

Figure 43 shows the same velocity data at a lower resolution of $3.488 \mu \mathrm{m}$ but with a larger overlap (same $872 \mathrm{~nm}$ vector spacing). $99.20 \%$ of the vectors are determined to be valid in the median test. The overall vector quality is high although there are still several spurious vectors near the leading edge. 


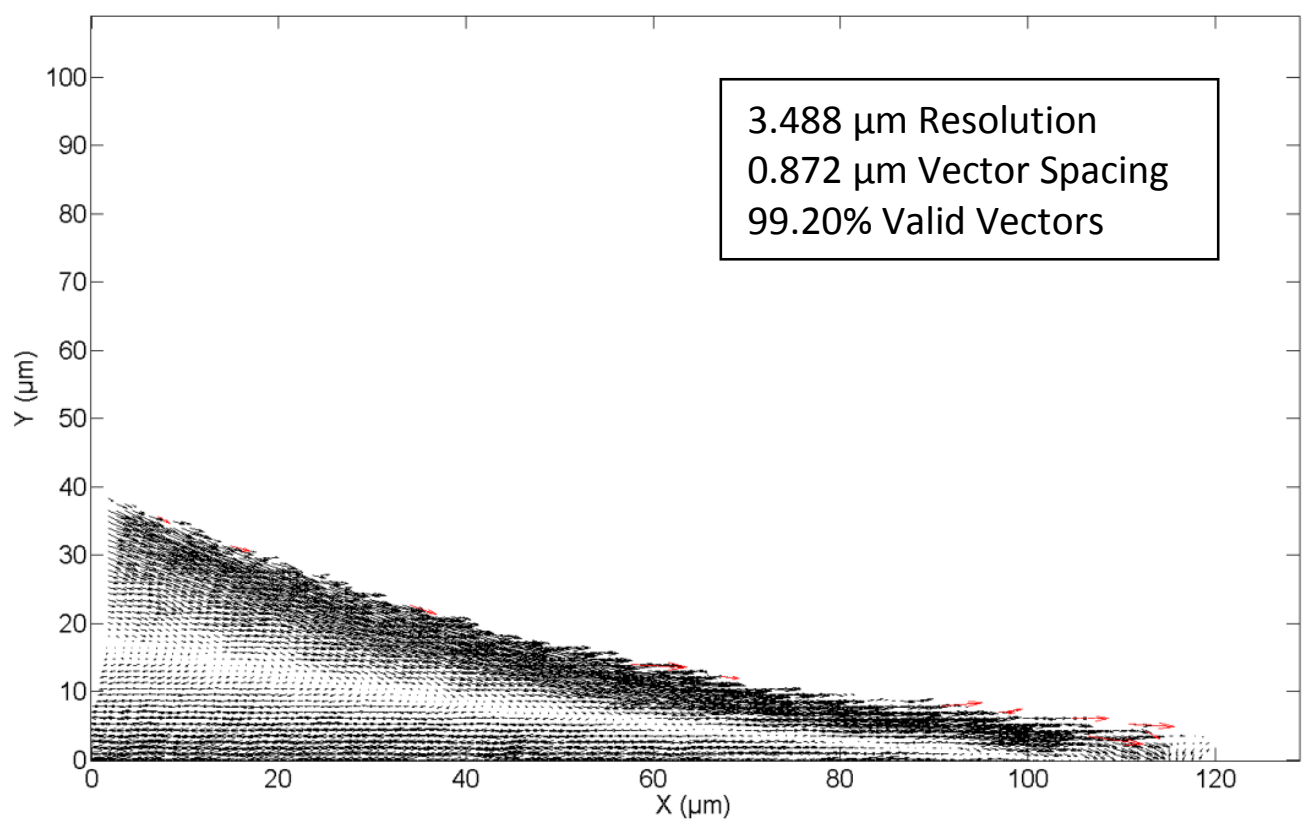

Figure $43 \mu \mathrm{PIV}$ velocity vectors for glycerol in a $30 \mu \mathrm{m} \times 300 \mu \mathrm{m}$ microchannel with $\mathrm{Ca}=1.9 \mathrm{E}-4.43$ pairs of images were analyzed using a 32 pixel interrogation region with a $75 \%$ overlap.

Figure 44 shows the velocity field when the resolution is increased to $1.744 \mu \mathrm{m}$ using a 16 pixel interrogation region with a 75\% overlap (vector spacing of $436 \mathrm{~nm}$ ). $98.25 \%$ of vectors are valid. Spurious vectors at the leading edge are reduced and there is a slight increase in spurious vectors along the interface. This occurs because the interrogation region is significantly less than the particle displacements for the highest fluid velocities found at the interface and along the solid boundary. The reduced interrogation size decreases the number of spurious vectors in the regions with reasonable velocities and high velocity gradients, making it more accurate near the liquid/gas/solid intersection. 


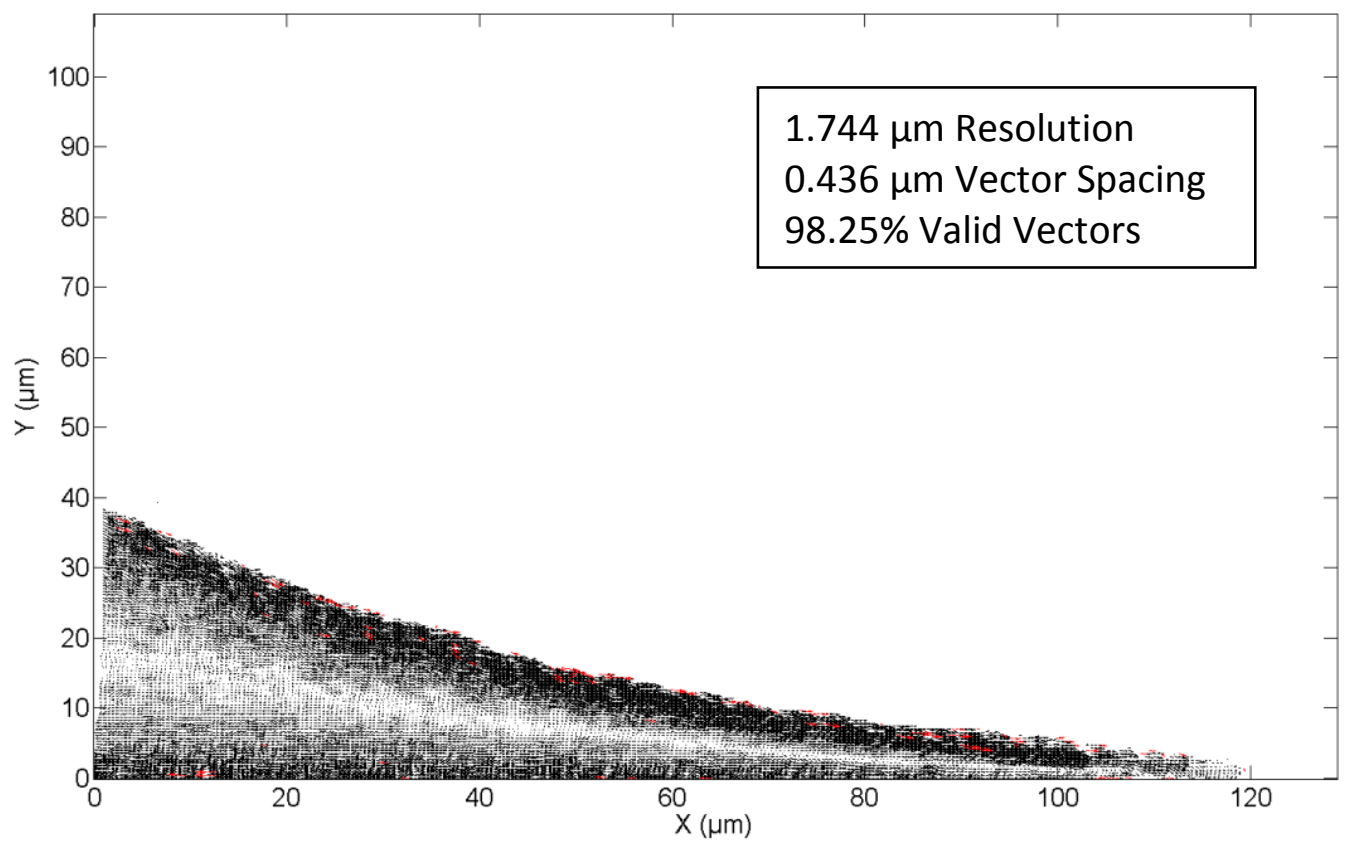

Figure $44 \mu \mathrm{PIV}$ velocity vectors for glycerol in a $30 \mu \mathrm{m} \times 300 \mu \mathrm{m}$ microchannel with $\mathrm{Ca}=1.9 \mathrm{E}-4.43$ pairs of images were analyzed using a 16 pixel interrogation region with a $75 \%$ overlap.

Figure 45 shows the results of an 8 pixel interrogation size. The velocity field has a resolution of $872 \mathrm{~nm}$ with a vector spacing of $218 \mathrm{~nm} .94 .11 \%$ of the vectors are valid. While this increase in resolution shows a decrease in valid velocity vectors, the majority of vectors within $10 \mu \mathrm{m}$ of the contact line are valid. Most of the spurious vectors occur in the high velocity regions near the interface and channel wall. 


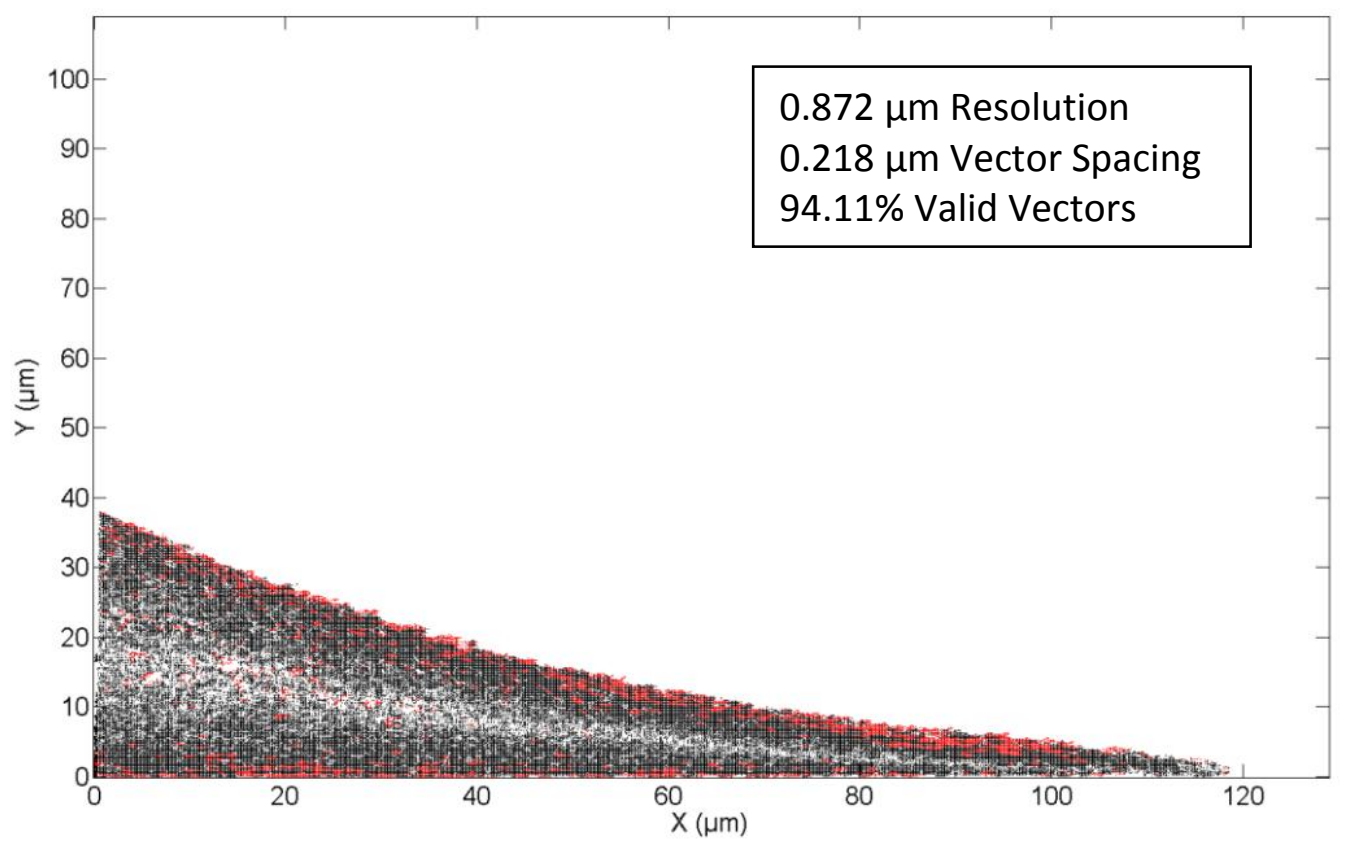

Figure $45 \mu \mathrm{PIV}$ velocity vectors for glycerol in a $30 \mu \mathrm{m}$ x $300 \mu \mathrm{m}$ microchannel with $\mathrm{Ca}=1.9 \mathrm{E}-4.43$ pairs of images were analyzed using a 8 pixel interrogation region with a $75 \%$ overlap.

Figure 46 and Figure 47 show a magnified region near the leading edge for the $1.744 \mu \mathrm{m}$ (Figure 44) and $872 \mathrm{~nm}$ (Figure 45) resolutions respectively. Velocity vectors near the leading edge are clearly seen. Very few spurious vectors appear in the $1.744 \mu \mathrm{m}$ resolution data. Spurious vectors are more than $15 \mu \mathrm{m}$ from the leading edge in the $872 \mathrm{~nm}$ resolution data. Flow patterns are clearly visible several hundred nanometers from the contact line. 


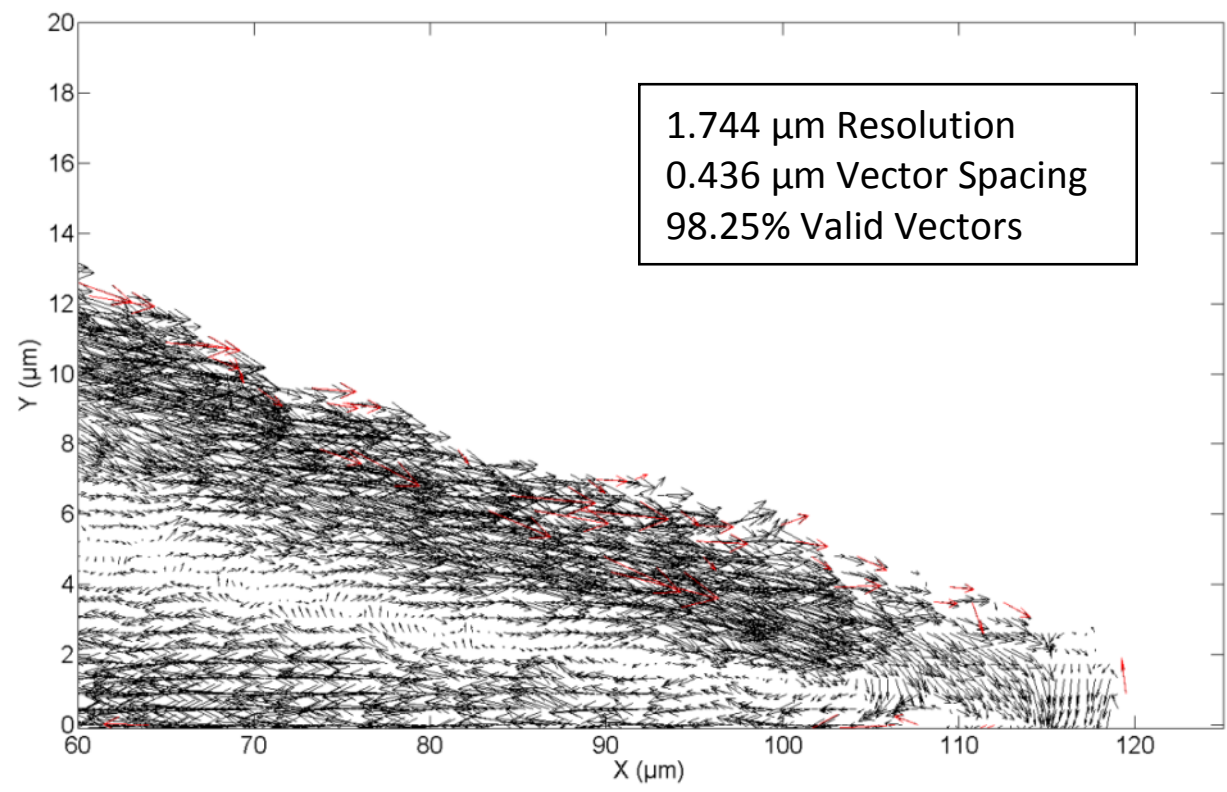

Figure $46 \mu$ PIV velocity vectors in a magnified region near the contact line. Glycerol in a $30 \mu \mathrm{m} \times 300$ $\mu \mathrm{m}$ microchannel with a 16 pixel interrogation region and a $75 \%$ overlap.

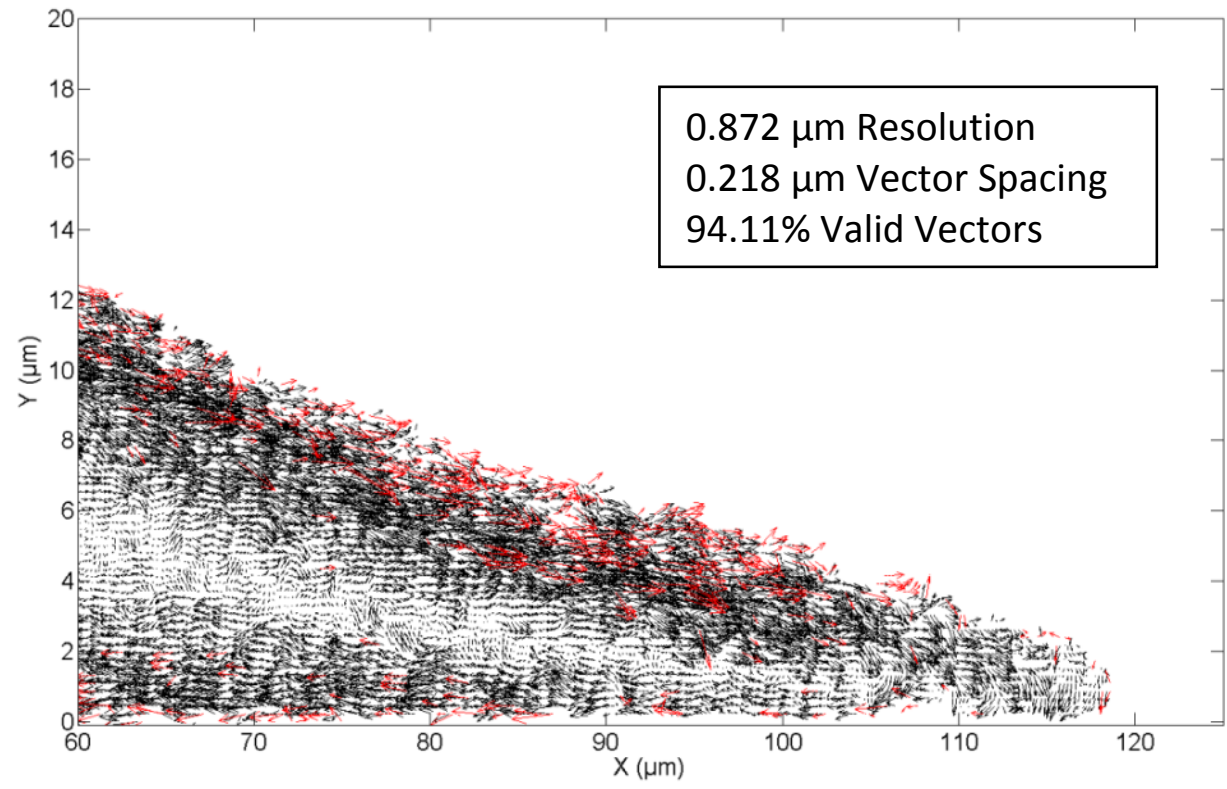

Figure $47 \mu$ PIV velocity vectors in a magnified region near the contact line. Glycerol in a $30 \mu \mathrm{m} \times 300$ $\mu \mathrm{m}$ microchannel with an 8 pixel interrogation region and a $75 \%$ overlap. 


\subsection{Summary of Experimental Results}

\subsubsection{Previous Experimental Limits}

The representative results of the nearly 100 experiments described above demonstrate the ability of the developed method to make simultaneous measurements of interface motion, dynamic contact angle, and local fluid velocities with $\mu$ PIV. Figure 48 and Figure 49 show the $872 \mathrm{~nm}$ resolution velocity field for glycerol in a $30 \mu \mathrm{m} \times 300 \mu \mathrm{m}$ channel and the previously published experimental limits. Dynamic contact angles and interface shape had been measured within $5 \mu \mathrm{m}$ of the contact line. Fluid velocities had been measured $30 \mu \mathrm{m}$ from the contact line. The presented results show fluid velocity measurements over two orders of magnitude closer to the contact line with an increase in resolution of over $3400 \%$.

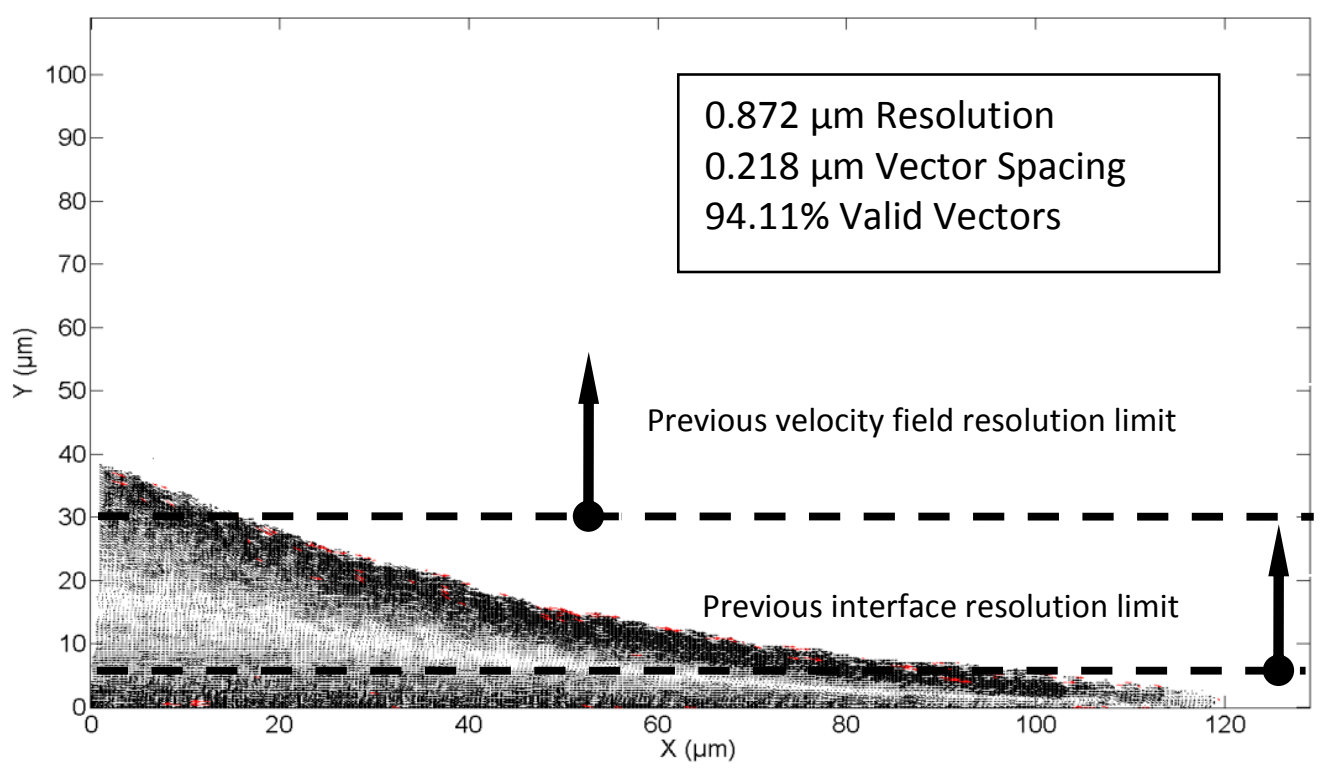

Figure 48 Previous measurement resolutions near the moving contact line. 


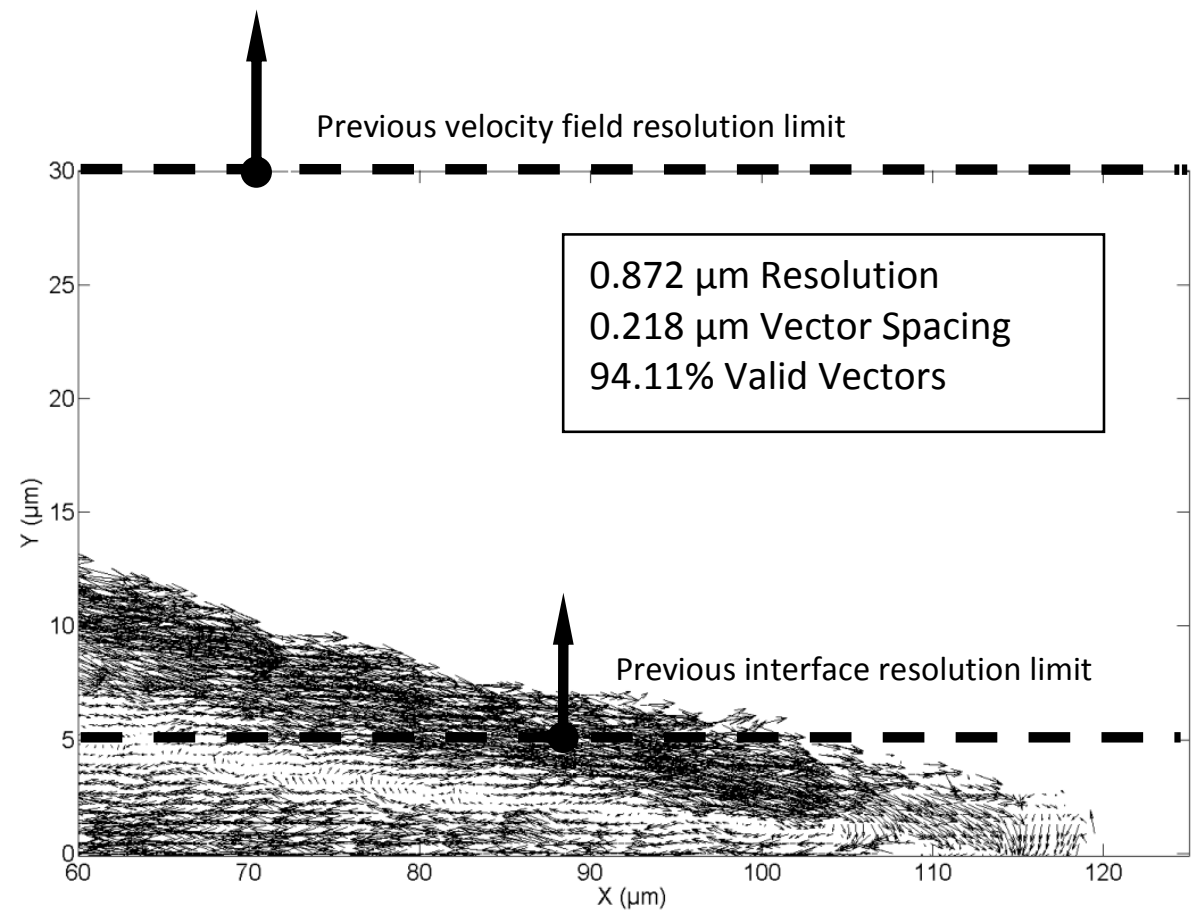

Figure 49 Previous measurement resolutions within $30 \mu \mathrm{m}$ of the moving contact line.

\subsubsection{Apparent Similarity to Theoretical Prediction}

Figure 50 shows the streamlines near the moving contact line for glycerol in a 30 $\mu \mathrm{m}$ by $300 \mu \mathrm{m}$ channel. Below previously published experimental limits is the appearance of a small recirculation zone. The vortex lies within $20 \mu \mathrm{m}$ of the leading edge less than $5 \mu \mathrm{m}$ from the wall. The appearance of such vortices had not been experimentally verified prior to this work. The recirculation bears a striking resemblance to the theoretical flow fields predicted by Y. Shikhmurzaev [1997], shown in the white inlay of Figure 50. The appearance of this recirculation zone in an 
experimental measurement with $98 \%$ valid vectors has potentially transformative implications to the current understanding of microscale fluid behavior. The vortex is far below previous experimental investigation and it is possible that deviation from theoretical models in the inner region may be caused by this undocumented flow behavior. While no definitive claims can be made regarding Shikhmurzaev's model, or the models of other theorists, the measurements do provide insight into the fundamental fluid behavior near a moving contact line that any valid model must incorporate. It also stresses the need to experimentally determine the actual fluid behavior near the moving contact line at the micro- and nano-scale.

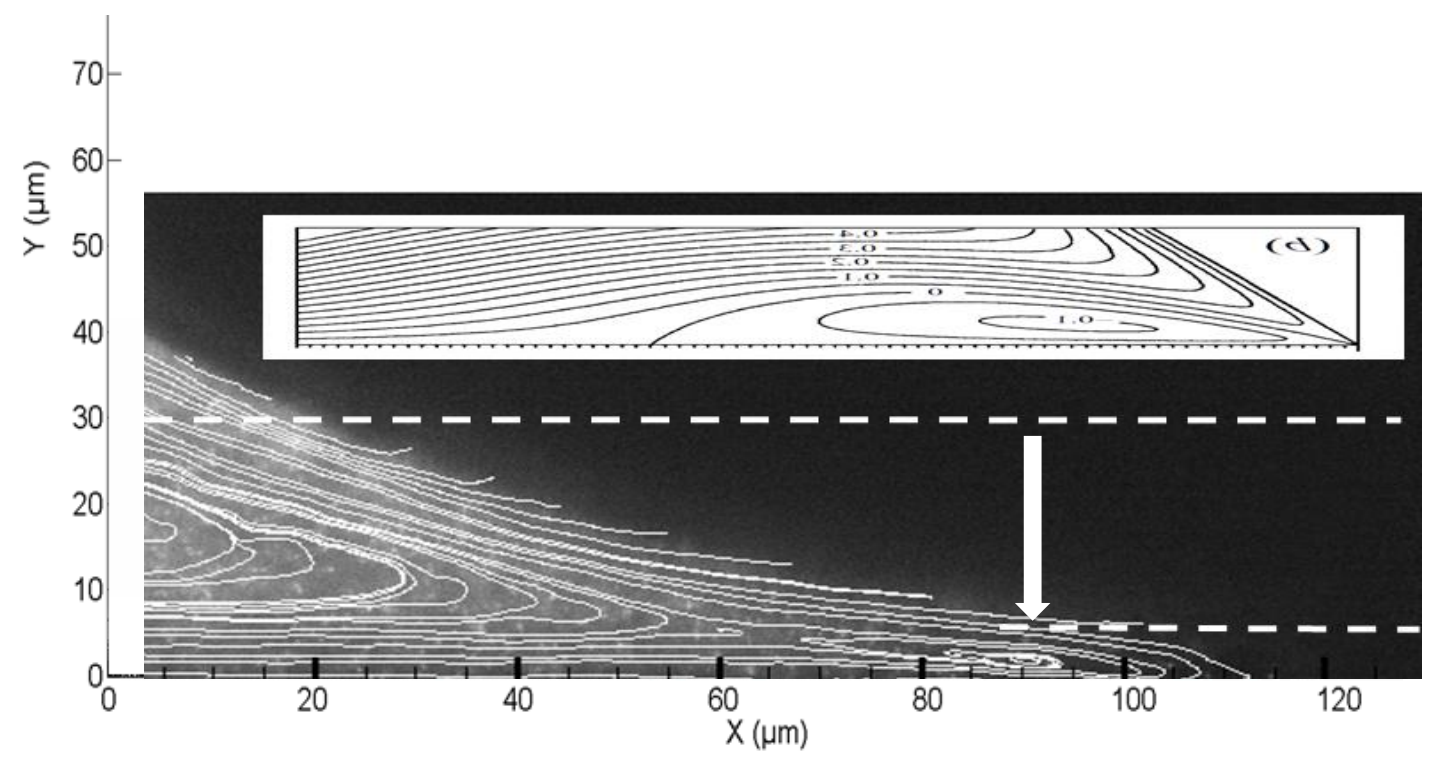

Figure 50 The appearance of a recirculation zone below previously published limits resembles the prediction of [Shikhmurzaev 1997]. 


\section{Extended Discussion \& Recommendations}

The method described above for the measurement of interface shape, dynamic contact angle, and local fluid velocities has greatly increased measurement resolution. However, the highest resolution results are extremely difficult to acquire due to poorly manufactured glass microchannels, relatively large fluorescent $\mu$ PIV particles, and the limits of linear stage motion. The limits of the technique are described below. These limits can be overcome in future work. Recommendations are provided.

\subsection{Glass Microchannels}

\subsubsection{Limits}

Borosilicate glass microchannels are made by an extrusion process. Channel dimensions vary significantly based on the quality of the die. While channel cross section is consistent along measurement lengths, corners are often rounded and walls are not perfectly flat. Figure 51 shows the cross section of a $50 \mu \mathrm{m} \times 500 \mu \mathrm{m}$ microchannel. While this channel is classified as rectangular, it has large corner radii and slightly curved surfaces. The radius of the corners is over $8 \mu \mathrm{m}$ in the channel shown. This curvature results in erroneous particle positions near the wall due to diffracted light. 

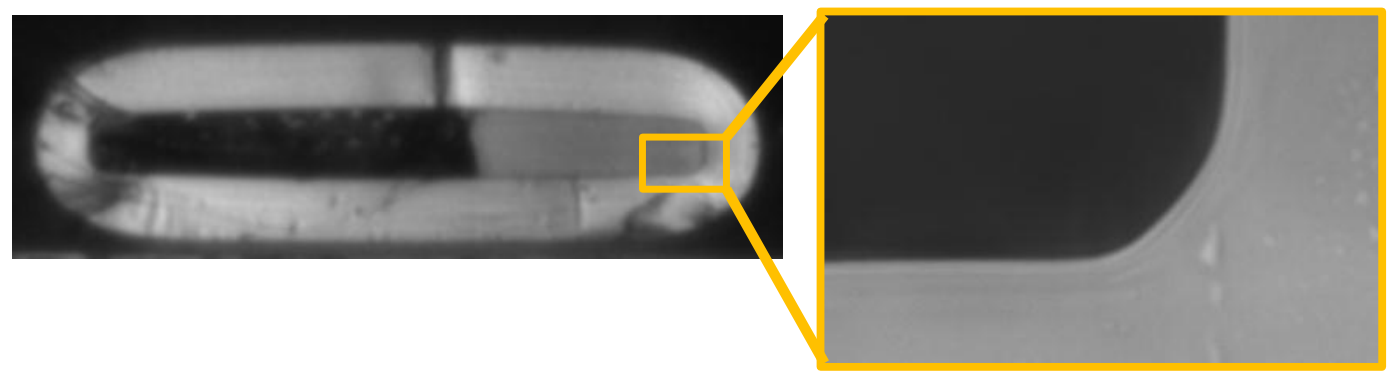

Figure 51 Corner radius in a "rectangular" glass microchannel.

In addition to corner radius, deformation in the channel walls lead to distorted interface shapes. Figure 52 shows the interface in a rectangular channel with straight walls (left) and concave walls (right). Deformation in interface shape complicates the comparison of measured quantities to theoretical models.
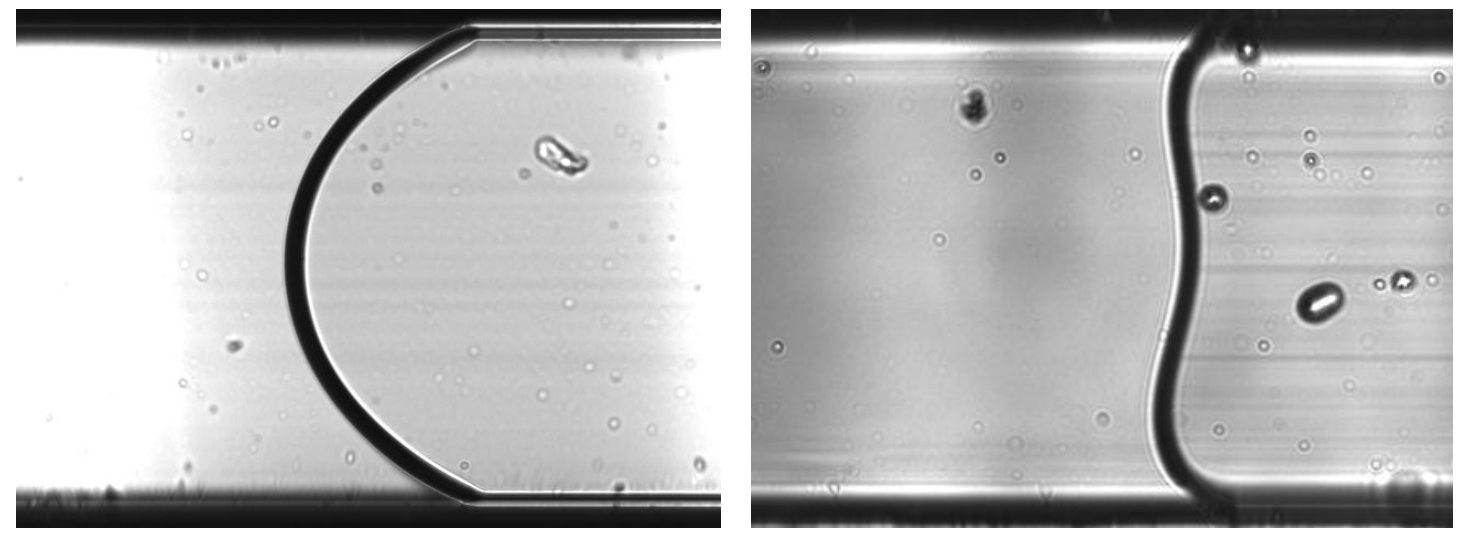

Figure 52 The meniscus in a rectangular channel with straight walls (left) and concave walls (right).

\subsubsection{Recommendations}

The most common method to manufacture precision microchannels and microfluidic devices is Deep Reactive lon Etching (DRIE). DRIE is a well-known 
fabrication technique capable of etching silicon and glass substrates [Clerc 1998]. Channel dimensions may have a wide range of aspect ratios with channel depths from several microns to several hundred microns. DRIE has shown good results with wall angles greater than $80^{\circ}$ and close to $90^{\circ}$ for shallow channel dimensions [Akashi 2006]. Examined using a scanning electron microscope (SEM), channel walls showed roughness of approximately $30 \mathrm{~nm}$ or less [Clerc 1998, Pike 2004]. DRIE is therefore a viable alternative to the extrusion process of currently available microchannels.

\section{$5.2 \mu \mathrm{PIV}$}

\subsubsection{Limits}

The limits of $\mu$ PIV were discussed herein. The theoretical limit using current hardware, and employing a single-pixel interrogation technique [Westerweel 2004], is $109 \mathrm{~nm}$. This resolution clearly exceeds the dimensions of the $275 \mathrm{~nm}$ fluorescent particles used currently. It is therefore beneficial to use a smaller particle in order to make velocity measurements closer to the moving contact line.

In addition to the size limit of the current $\mu$ PIV method, test fluids are limited to aqueous solutions by the properties of the fluorescent particles. $\mu$ PIV particles are not compatible with the non-aqueous liquids typically used in the study of capillary flow, the most common of which is PDMS oil (polydimethylsiloxane). PDMS is desirable because it is perfectly wetting on glass and silicon, it is inert, and has very 
well-documented fluid properties. In contrast, water is a polar fluid which leads to easy contamination and electrostatic effects, and glycerol and GFS have strongly temperature dependent properties.

\subsubsection{Recommendations}

A relatively new yet promising particle is the quantum dot (QD). Quantum dots range in size from $5 \mathrm{~nm}$ to $20 \mathrm{~nm}$ in diameter. Quantum dots have a higher quantum yield than conventional fluorescent particles, making them significantly brighter. Quantum dots are more photostable and do not photobleach when exposed to excitation wavelengths for extended periods. Experiments in the Microscale Laboratory have successfully dispersed $8 \mathrm{~nm}$ CdSe/ZnS (cadmium selenide core with a zinc sulfide shell) quantum dots (620 nm emission wavelength, Ocean NanoTech, Springdale Arkansas) in solvents and PDMS oil. The excitation wavelength of $\mathrm{CdSe} / \mathrm{ZnS}$ quantum dots is in the same range as the $\mu \mathrm{PIV}$ lasers and the emission wavelength is tuned to match current optical filters. The use of quantum dots as tracer particles for $\mu$ PIV is currently being explored. Freudenthal et al. [2007] created a $70 \mathrm{~nm}$ quantum nanosphere conjugating QDs to a polystyrene sphere. They developed the particle for use in low cost $\mu$ PIV systems with low power lasers; however the technique shows the viability of quantum dots in developing a high 
resolution $\mu \mathrm{PIV}$ particle substitute capable of measuring flow velocities in nonaqueous fluids.

\subsection{Automated Stage Motion}

\subsubsection{Limits}

One of the biggest challenges in acquiring a sufficient number of quality $\mu \mathrm{PIV}$ images is keeping the interface at a constant location in the field of view. While a linear approximation is accurate to within reasonable lengths over several seconds, large sets of image pairs take minutes to acquire, thereby limiting the current method to between 25 and 250 pairs. Much higher quality velocity fields require a more accurate stage motion control that follows the true motion of the fluid interface which decreases in velocity with $\mathrm{t}^{1 / 2}$.

\subsubsection{Recommendations}

The automated stage may be controlled by a LabVIEW virtual instrument (VI). A VI is currently being developed by Ashford Solutions (Portland, OR) that enables a functional stage velocity to be programmed on the fly based on current joystick speed. The interface is positioned manually in the field of view and the $\mathrm{VI}$ continues at the prescribed decreasing rate. This method will enable 100 s or perhaps 1000 s of images to be captured during a single experiment. In future iterations of the LabVIEW 
VI being developed, real-time image analysis may enable autonomous interface tracking. Automated control is a necessary addition in order to reduce error from the current method. 


\section{Conclusion}

\subsection{Overview of Developed Methods}

An experimental method has been developed to simultaneously measure interface motion, dynamic contact angles, and local fluid velocity fields using $\mu$ PIV. The technique is capable of exploring near the inner region of the moving contact line with a resolution of less than $1 \mu \mathrm{m}$ at distances of several hundred nanometers from the interface. This unprecedented resolution has the ability to reveal flow behavior either unidentified or unconfirmed by the many theorists working to describe the important physics of the wetting process.

\subsection{Overview of Results}

Experiments have been conducted for glycerol and GFS in $30 \mu \mathrm{m} \times 300 \mu \mathrm{m}, 50 \mu \mathrm{m}$ $\times 500 \mu \mathrm{m}$, and $100 \mu \mathrm{m} \times 1000 \mu \mathrm{m}$ glass microchannels. Interface velocities have been measured between $1.7 \mu \mathrm{m} / \mathrm{s}$ and $33 \mu \mathrm{m} / \mathrm{s}$. Dynamic contact angles have been manually measured between $1.1 \mu \mathrm{m}$ and $120 \mu \mathrm{m}$ from the contact line, and calculated from $\mu$ PIV data to within several hundred nanometers from the contact line. Average dynamic contact angles range from $12.2^{\circ} \pm 3.7^{\circ}$ to $40.2^{\circ} \pm 2.7^{\circ}$, while apparent contact angles at the leading edge exceed $55^{\circ}$. This deviation from the geometrically dependent shape of the meniscus shows the promise of the ability to measure angles near the inner region. Capillary numbers ranged from $1.7 \times 10^{-4}$ to 
$6.2 \times 10^{-4}$. The appearance of a recirculation zone far below previously published experimental limits demonstrates the power and significance of the method as well as confirming the necessity to provide high resolution measurements to theorists.

\subsection{Contributions to the Study of Contact Line Motion}

The pace of theoretical work has extended far beyond the limits of empirical measurement in the study of contact line motion. Our understanding of dynamic wetting has been stunted by the inability to experimentally explore the inner region of the contact line where current theoretical models break down. Previous methods were limited to the outer and intermediate regions and could not provide experimental data at a resolution capable of differentiating between competing theories. The developed method and the results provided demonstrate a potentially significant contribution to the study of wetting phenomena and passive fluid transport by narrowing the gap between the theoretical models and experimental observation. Continued work to refine the experimental technique and expand the range of flow configurations may ultimately provide a benchmark data set by which theoretical models may be validated, refined, and expanded to better model a process so common in nature and industry. 


\section{References}

Akashi, T., \& Yoshimura, Y. (2006). Deep reactive ion etching of borosilicate glass using an anodically bonded silicon wafer as an etching mask. Journal of Micromechanics and Microengineering, 16, 1051.

Bertozzi, A. (1998). The mathematics of moving contact lines in thin liquid films. Notices AMS, 45, 689-697.

Billingham, J. (2006). On a model for the motion of a contact line on a smooth solid surface. European Journal of Applied Mathematics, 17(03), 347-382.

Blake, T. (2006). The physics of moving wetting lines. Journal of colloid and interface science, 299(1), 1-13.

Blake, T., \& Shikhmurzaev, Y. (2002). Dynamic wetting by liquids of different viscosity. Journal of colloid and interface science, 253(1), 196-202.

Blake, T., Bracke, M., \& Shikhmurzaev, Y. (1999). Experimental evidence of nonlocal hydrodynamic influence on the dynamic contact angle. Physics of fluids, 11, 1995.

Bonn, D., Eggers, J., Indekeu, J., Meunier, J., \& Rolley, E. (2009). Wetting and spreading. Reviews of modern physics, 81(2), 739. 
Bourdon, C., Olsen, M., \& Gorby, A. (2004). Validation of an analytical solution for depth of correlation in microscopic particle image velocimetry. Measurement Science and Technology, 15, 318.

Bourdon, C., Olsen, M., \& Gorby, A. (2006). The depth of correlation in micro-PIV for high numerical aperture and immersion objectives. Journal of fluids engineering, 128(4), 883-886.

Brochard-Wyart, F., \& Gennes, P. D. (1992). Dynamics of partial wetting. Advances in colloid and interface science, 39, 1-11.

Chan, W., \& Yang, C. (2005). Surface-tension-driven liquid--liquid displacement in a capillary. Journal of Micromechanics and Microengineering, 15, 1722.

Chen, Q., Ramé, E., \& Garoff, S. (1997). The velocity field near moving contact lines. Journal of Fluid Mechanics, 337, 49-66.

Clerc, P., Dellmann, L., Grétillat, F., Grétillat, M., Indermühle, P., Jeanneret, S., et al. (1998). Advanced deep reactive ion etching: a versatile tool for microelectromechanical systems. Journal of Micromechanics and Microengineering, 8, 272.

Coninck, J. D., \& Blake, T. (2008). Wetting and molecular dynamics simulations of simple liquids. Annu. Rev. Mater. Res., 38, 1-22. 
Cox, R. (1986). The dynamics of the spreading of liquids on a solid surface. Part 1. Viscous flow. Journal of Fluid Mechanics, 168(1), 169-194.

Dong, M., \& Chatzis, I. (1995). The imbibition and flow of a wetting liquid along the corners of a square capillary tube. Journal of colloid and interface science, 172(2), 278-288.

Dussan, E. (1979). On the spreading of liquids on solid surfaces: static and dynamic contact lines. Annual Review of Fluid Mechanics, 11(1), 371-400.

Dussan, V., Ramé, E., \& Garoff, S. (1991). On identifying the appropriate boundary conditions at a moving contact line: an experimental investigation. Journal of Fluid Mechanics, 230(1), 97-116.

Eggers, J., \& Stone, H. (2004). Characteristic lengths at moving contact lines for a perfectly wetting fluid: the influence of speed on the dynamic contact angle. Journal of Fluid Mechanics, 505, 309-321.

Freudenthal, P., Pommer, M., Meinhart, C., \& Piorek, B. (2007). Quantum nanospheres for sub-micron particle image velocimetry. Experiments in Fluids, 43(4), 525-533.

Fuentes, J., \& Cerro, R. (2005). Flow patterns and interfacial velocities near a moving contact line. Experiments in fluids, 38(4), 503-510. 
Gennes, P. d. (1985). Wetting: statics and dynamics. Reviews of Modern Physics, $57(3), 827$.

Huh, C., \& Scriven, L. (1971). Hydrodynamic model of steady movement of a solid/liquid/fluid contact line. Journal of Colloid and Interface Science, 35(1), 85-101.

Ichikawa, N., Hosokawa, K., \& Maeda, R. (2004). Interface motion of capillary-driven flow in rectangular microchannel. Journal of colloid and interface science, $280(1), 155-164$.

Jong, W., Kuo, T., Ho, S., Chiu, H., \& Peng, S. (2007). Flows in rectangular microchannels driven by capillary force and gravity. International communications in heat and mass transfer, 34(2), 186-196.

Kim, E., \& Whitesides, G. (1997). Imbibition and flow of wetting liquids in noncircular capillaries. The Journal of Physical Chemistry B, 101(6), 855-863.

Kim, W., Tretheway, D., \& Kohles, S. (2009). An inverse method for predicting tissuelevel mechanics from cellular mechanical input. Journal of biomechanics, 42(3), 395-399.

Kistler, S. (1993). Hydrodynamics of wetting. Wettability, 311-430. 
Lindken, R., Rossi, M., Gro, S., \& Westerweel, J. (2009). Micro-Particle Image Velocimetry ([small micro] PIV): Recent developments, applications, and guidelines. Lab on a Chip, 9(17), 2551-2567.

Lindner-Silwester, T., \& Schneider, W. (2006). Authors' reply to the Remark on "The moving contact line with weak viscosity effects--an application and evaluation of Shikhmurzaev's model". Acta Mechanica, 182(1), 145-146.

Marsh, J., Garoff, S., \& V, E. D. (1993). Dynamic contact angles and hydrodynamics near a moving contact line. Physical review letters, 70(18), 2778-2781.

Meinhart, C., \& Wereley, S. (2003). The theory of diffraction-limited resolution in microparticle image velocimetry. Measurement science and technology, 14, 1047.

Meinhart, C., Wereley, S., \& Santiago, J. (1999). PIV measurements of a microchannel flow. Experiments in Fluids, 27(5), 414-419.

Meinhart, C., Wereley, S., \& Santiago, J. (2000). A PIV algorithm for estimating timeaveraged velocity fields. Transactions-American Society of Mechanical Engineers Journal of Fluids Engineering, 122(2), 285-289.

Melling, A. (1997). Tracer particles and seeding for particle image velocimetry. Measurement Science and Technology, 8, 1406. 
Nève, N., Kohles, S., Winn, S., \& Tretheway, D. (2010). Manipulation of Suspended Single Cells by Microfluidics and Optical Tweezers. Cellular and molecular bioengineering, 3(3), 213-228.

Nève, N., Lingwood, J., Zimmerman, J., Kohles, S., \& Tretheway, D. (2008). The \$MU\$PIVOT: an integrated particle image velocimeter and optical tweezers instrument for microenvironment investigations. Measurement Science and Technology, 19, 095403.

Olsen, M., \& Adrian, R. (2000). Brownian motion and correlation in particle image velocimetry. Optics I\& Laser Technology, 32(7-8), 621-627.

Olsen, M., \& Adrian, R. (2000). Out-of-focus effects on particle image visibility and correlation in microscopic particle image velocimetry. Experiments in Fluids, $29,166-174$.

Petrov, P., \& Petrov, I. (1992). A combined molecular-hydrodynamic approach to wetting kinetics. Langmuir, 8(7), 1762-1767.

Pike, W., Karl, W., Kumar, S., Vijendran, S., \& Semple, T. (2004). Analysis of sidewall quality in through-wafer deep reactive-ion etching. Microelectronic engineering, 73, 340-345. 
Prasad, A., \& Adrian, R. (1993). Stereoscopic particle image velocimetry applied to liquid flows. Experiments in fluids, 15(1), 49-60.

Qian, T., Wang, X., \& Sheng, P. (2006). A variational approach to moving contact line hydrodynamics. Journal of Fluid Mechanics, 564(1), 333-360.

Qian, T., Wang, X., \& Sheng, P. (2006). Molecular hydrodynamics of the moving contact line in two-phase immiscible flows. Commun. Comput. Phys, 1(1), 152.

Qian, T., Wu, C., Lei, S., Wang, X., \& Sheng, P. (2009). Modeling and simulations for molecular scale hydrodynamics of the moving contact line in immiscible twophase flows. Journal of Physics: Condensed Matter, 21, 464119.

Ralston, J., Popescu, M., \& Sedev, R. (2008). Dynamics of wetting from an experimental point of view. Annu. Rev. Mater. Res., 38, 23-43.

Ramé, E. (2002). Moving Contact Line Problem: State of the Contact Angle Boundary Condition. Encyclopedia of Surface and Colloid Science. Marcel Dekker, New York.

Ransohoff, T., \& Radke, C. (1988). Laminar flow of a wetting liquid along the corners of a predominantly gas-occupied noncircular pore. Journal of colloid and interface science, 121(2), 392-401. 
Ruijter, M. D., Blake, T., \& Coninck, J. D. (1999). Dynamic wetting studied by molecular modeling simulations of droplet spreading. Langmuir, 15(22), 78367847.

Santiago, J., Wereley, S., Meinhart, C., Beebe, D., \& Adrian, R. (1998). A particle image velocimetry system for microfluidics. Experiments in Fluids, 25(4), 316-319.

Savelski, M., Shetty, S., Kolb, W., \& Cerro, R. (1995). Flow patterns associated with the steady movement of a solid/liquid/fluid contact line. Journal of colloid and interface science, 176(1), 117-127.

Shankar, P., \& Kumar, M. (1994). Experimental determination of the kinematic viscosity of glycerol-water mixtures. Proceedings: Mathematical and Physical Sciences, $573-581$.

Shen, C., \& Ruth, D. (1998). Experimental and numerical investigations of the interface profile close to a moving contact line. Physics of Fluids, 10, 789.

Shikhmurzaev, Y. (1993). The moving contact line on a smooth solid surface. International journal of multiphase flow, 19(4), 589-610.

Shikhmurzaev, Y. (1997). Moving contact lines in liquid/liquid/solid systems. Journal of Fluid Mechanics, 334(1), 211-249. 
Shikhmurzaev, Y. (2006). Remark on "The moving contact line with weak viscosity effects--an application and evaluation of Shikhmurzaev's model" by T. Lindner-Silwester and W. Schneider (Acta Mech. 176, 245--258, 2005). Acta Mechanica, 182(1), 141-143.

Shikhmurzaev, Y., \& Blake, T. (2004). Response to the comment on [J. Colloid Interface Sci. 253 (2002) 196] by J. Eggers and R. Evans. Journal of Colloid and Interface Science, 280(2), 539-541.

Telis, V., Telis-Romero, J., Mazzotti, H., \& Gabas, A. (2007). Viscosity of aqueous carbohydrate solutions at different temperatures and concentrations. International Journal of Food Properties, 10(1), 185-195.

Tretheway, D., \& Meinhart, C. (2002). Apparent fluid slip at hydrophobic microchannel walls. Physics of Fluids, 14, L9.

Vennemann, P., Kiger, K., Lindken, R., Groenendijk, B., Vos, S. S.-d., Hagen, T. t., et al. (2006). In vivo micro particle image velocimetry measurements of bloodplasma in the embryonic avian heart. Journal of biomechanics, 39(7), 11911200.

Voinov, O. (1976). Hydrodynamics of wetting. Fluid Dynamics, 11(5), 714-721. 
Weislogel, M., \& Lichter, S. (1998). Capillary flow in an interior corner. Journal of Fluid Mechanics, 373, 349-378.

Wereley, S., \& Meinhart, C. (2010). Recent advances in micro-particle image velocimetry. Annual Review of Fluid Mechanics, 42, 557-576.

Wereley, S., Gui, L., \& Meinhart, C. (2002). Advanced algorithms for microscale particle image velocimetry. AIAA journal, 40(6), 1047-1055.

Westerweel, J., \& Scarano, F. (2005). Universal outlier detection for PIV data. Experiments in Fluids, 39(6), 1096-1100.

Westerweel, J., Geelhoed, P., \& Lindken, R. (2004). Single-pixel resolution ensemble correlation for micro-PIV applications. Experiments in fluids, 37(3), 375-384.

Williams, S., Park, C., \& Wereley, S. (2010). Advances and applications on microfluidic velocimetry techniques. Microfluidics and Nanofluidics, 8(6), 709-726.

Wilson, M., Summers, J., Shikhmurzaev, Y., Clarke, A., \& Blake, T. (2006). Nonlocal hydrodynamic influence on the dynamic contact angle: Slip models versus experiment. Physical Review E, 73(4), 041606.

Yang, L., Yao, T., \& Tai, Y. (2004). The marching velocity of the capillary meniscus in a microchannel. Journal of Micromechanics and Microengineering, 14, 220. 
Zhu, Y., \& Petkovic-Duran, K. (2010). Capillary flow in microchannels. Microfluidics and Nanofluidics, 8(2), 275-282. 


\section{Appendix}

The following code is used to convert the raw $\mu$ PIV data output from CleanVec, detect spurious vectors, calculate vector angles, and plot the results. 'PIV_Residual' is the main function. The input of 'PIV_Residual' is a matrix comprised of a list of vectors of the form $[x, y, U, V]$. 'Get UV' transforms the list of vectors into two matrices $U$ and $V$, with size equal to the number of coordinates in the $[x, y, U, V]$ data set. 'Get info1' was adapted from [Westerweel 2005] to detect spurious vectors. The percentage of valid vectors is displayed and a matrix called 'info1' is created where a 0 represents a valid vector and a 1 represents a spurious vector. The 'info1' data is sent to the 'Get Plot' function. 'Get Plot' creates a vector plot with valid vectors in black and spurious vectors in red, which is determined by the 'info1' matrix. In order to hide empty vectors, values of 0 are displayed in white. 'Get Plot' also creates a contour plot of the vector angles to local provide dynamic contact angle information. The user can set axis limits, vector length, and channel wall location for both plots.

(Developed with help from Alex Baker, Gerry Recktenwald, and Ben Furst.)

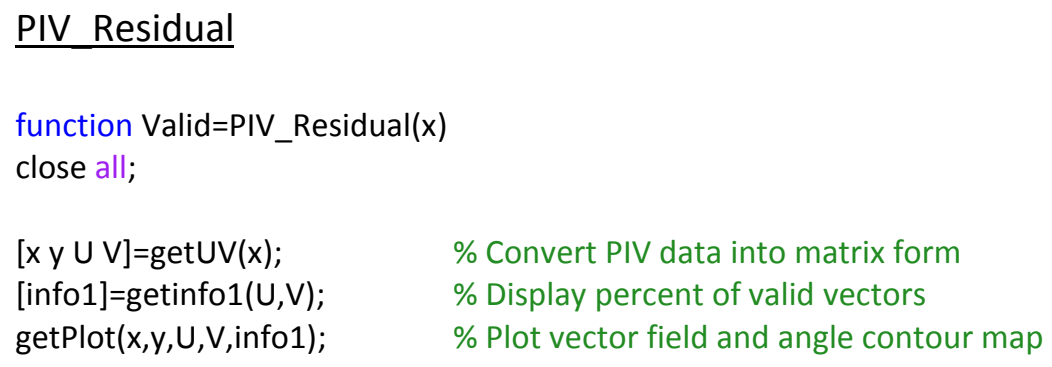




\section{Get UV}

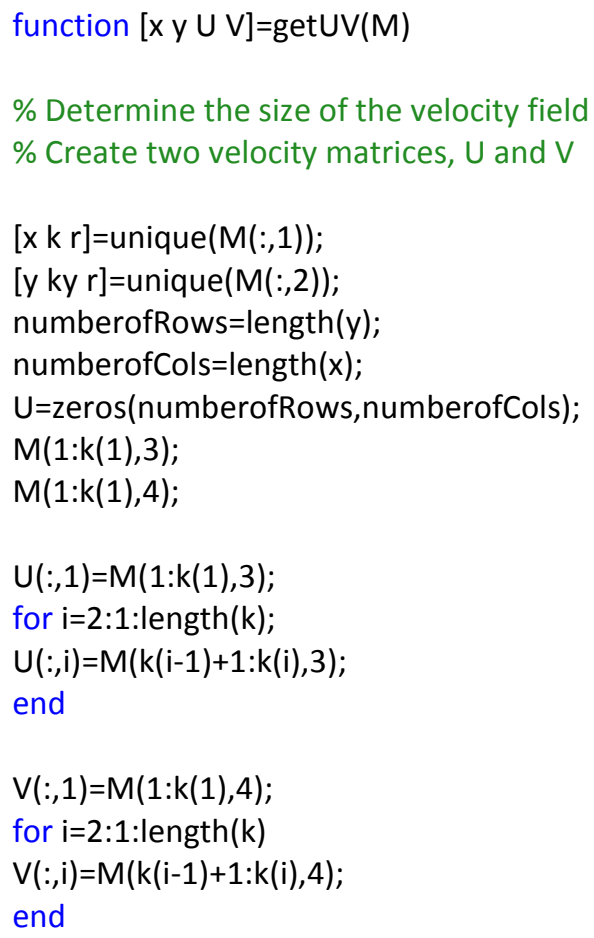

\section{Get info1}

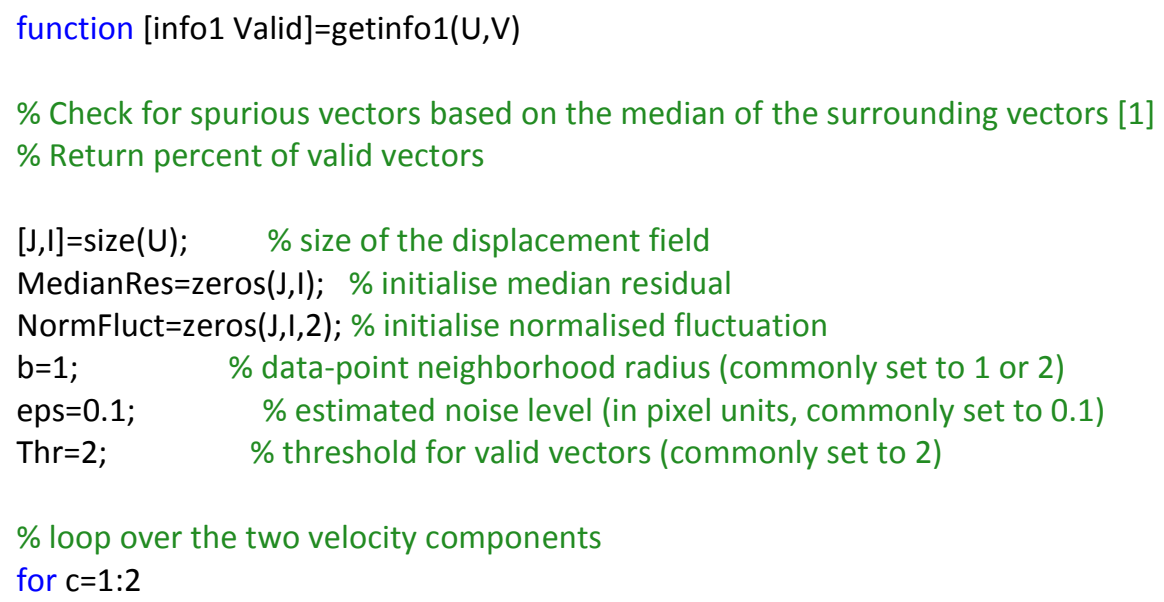




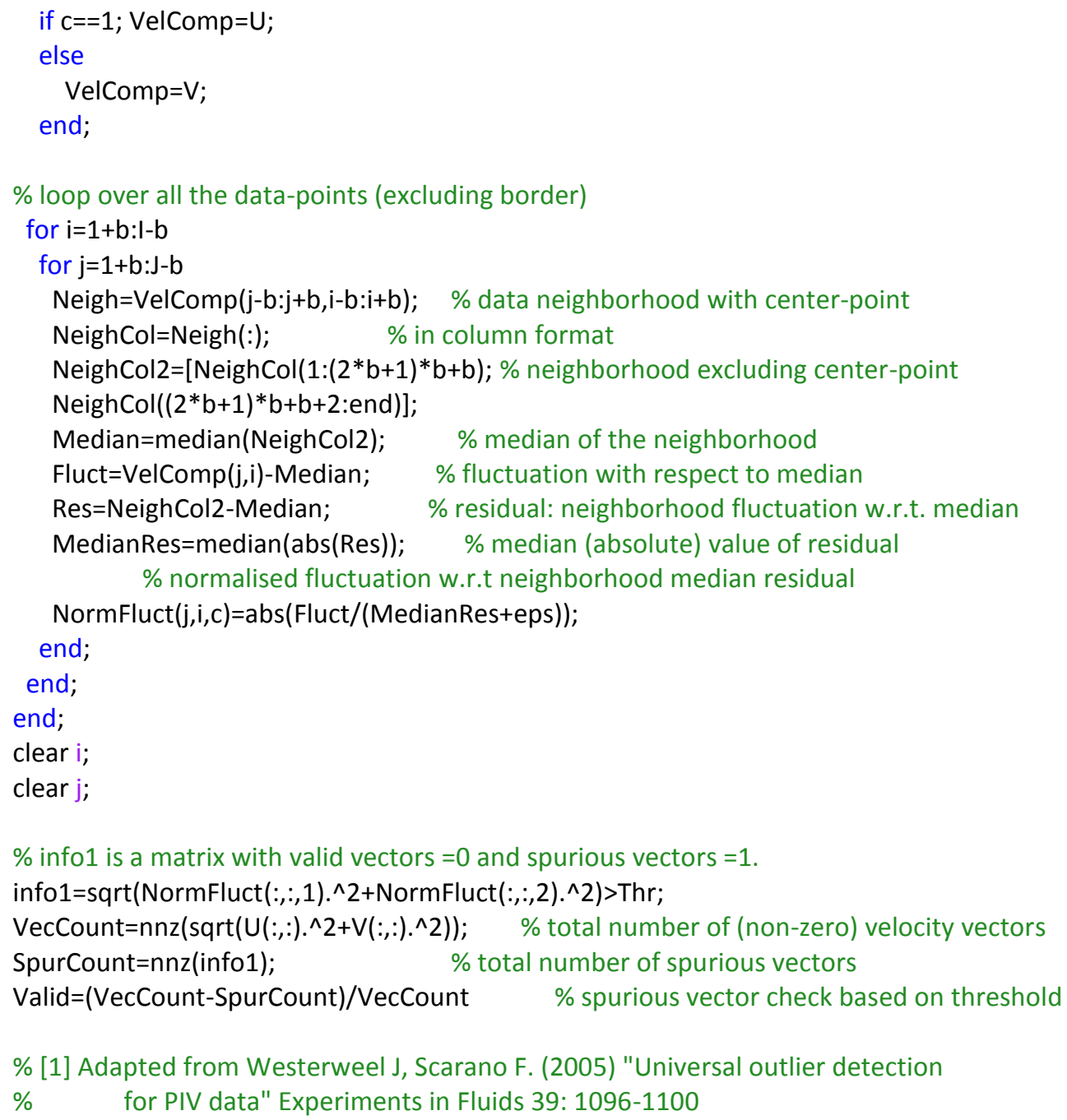

\title{
Get Plot
}

\author{
function getPlot( $x, y, U, V$,info1) \\ $\%$ Display vector plot and contour plot \\ Scale=5; \% Set vector length \\ Wall=0; \% Set wall distance in field of view (in pixels)

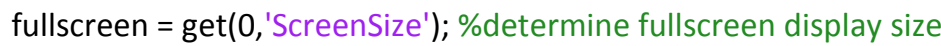




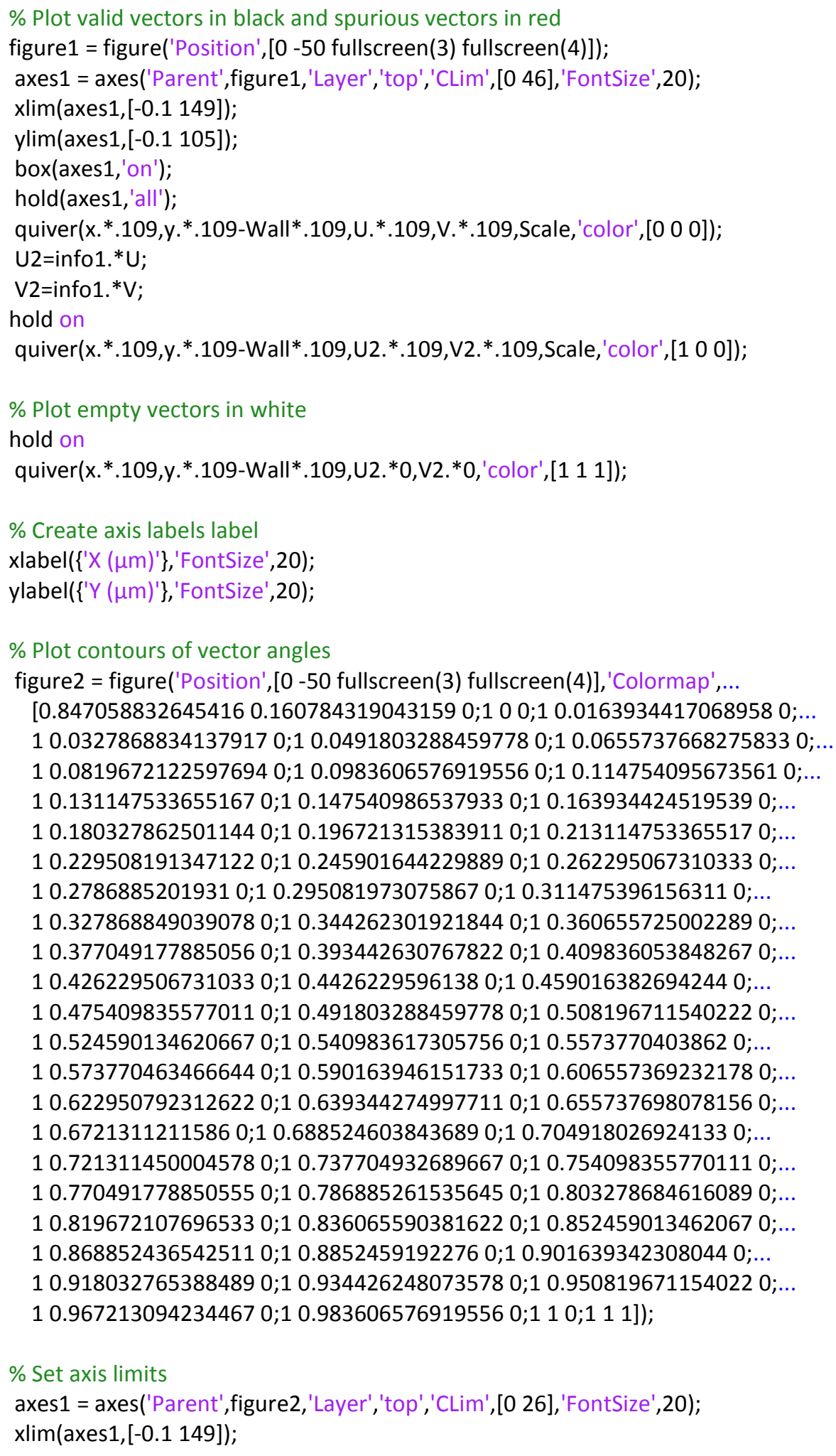




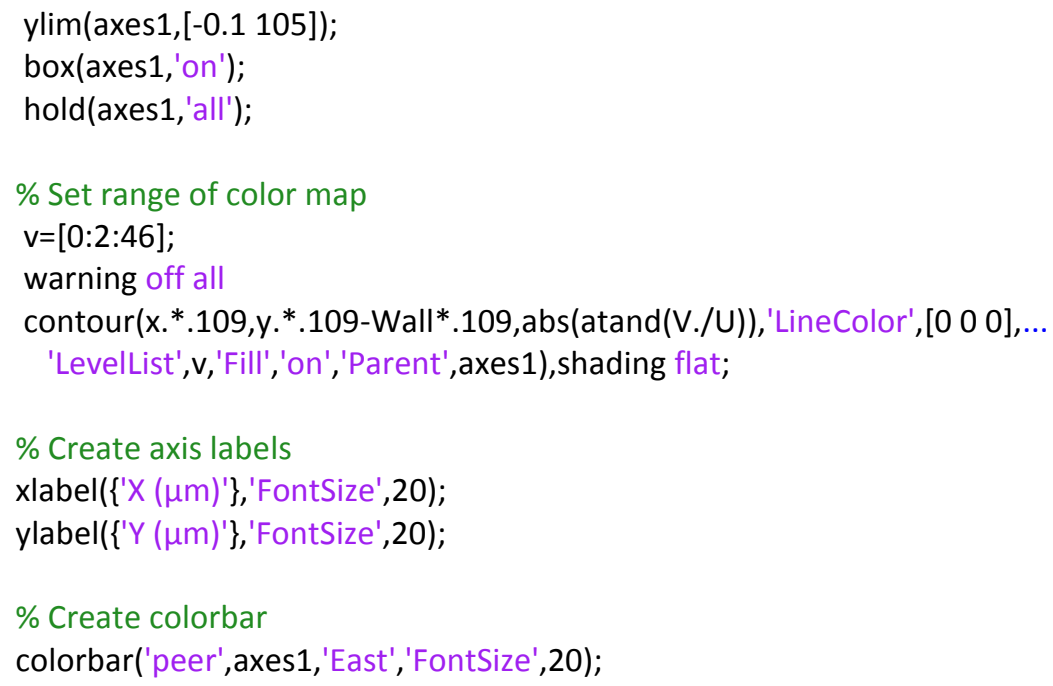

